\begin{abstract}
YORK, ALEXANDER. Experimental Investigation of Rate-Dependent Inner Hysteresis Loops in PZT

The rate-dependence of piezoelectric material resulting from the kinetics of domain switching is an important factor that needs to be included in realistic modeling attempts. This paper provides a systematic study of the rate-dependent hysteresis behavior of a commercially available PZT stack actuator. Experiments covering full loop as well as first, second, third and forth order reversals are conducted at different loading rates with polarization and strain recorded. Creep behavior at different constant levels of the electric field is also observed. The kinetics are characterized by strongly varying relaxation times that can be associated with different switching mechanisms. Inner hysteresis loops in the $(\mathrm{P}, \mathrm{E})$ diagram are also shown to have different characteristics depending on the electric field level at the reversal points.
\end{abstract}




\title{
EXPERIMENTAL INVESTIGATION OF RATE-DEPENDENT INNER HYSTERESIS LOOPS IN PZT
}

\author{
BY \\ Alexander York \\ A THESIS SUbMitTed TO THE GRADUATE FACUlTy OF \\ NORTH CAROLINA STATE UNIVERSITY \\ IN PARTIAL FULFILLMENT OF THE \\ REQUIREMENTS FOR THE DEGREE OF \\ MASTER OF SCIENCE \\ DEPARTMENT OF MECHANICAL AND AEROSPACE ENGINEERING \\ RALEIGH \\ APRIL 2005
}

APPROVED BY:

CHAIR OF ADVISORY COMMITTEE 
To my future wife, Laura Higbie York 


\title{
Biography
}

\begin{abstract}
Alexander York was born in New York, April 3, 1980. He attended the public schools until high school where he enrolled at Fordham Preparatory School. In 1998 he enrolled at Kenyon College and received a Bachelor of Arts in 2002 majoring in Physics with a minor in Mathematics. In 2003 he enrolled in the Mechanical and Aerospace Engineering program at North Carolina State University as a masters student. He is still alive today.
\end{abstract}




\section{Acknowledgments}

First, I would like to acknowledge my advisor Dr. Seelecke for his guidance and patience. I would also like to acknowledge the other members of the Adaptive Structures Lab at NCSU especially Olaf Heintze and Qifu Li. Thank you Olaf for all your advise and help in all my experimental hurtles, we will miss you when you return home to Germany.

I would like to thank my committee members, Dr. Gregory Buckner, and Dr. M. K. Ramasubramanian for supporting me and being on my committee.

For designing the experimental setup and advising me through the start of my research, I would like to thank Jinghua Zhong for all his work.

I would like to acknowledge my friends and classmates here in the MAE department at NCSU, Alex Paleocrassis, Wassim Azzi, James Lorek, Ryan Dennis, and Jason Dilwith. Your support, inspiration and friendship has made the last two years a truly memorable experience.

I would like to thank my parents, Daria York and Christopher York. Your love and support has made my success possible.

Finally, I would like to acknowledge my fiancee, Laura. You mean the world to me. Thank you for your support and understanding through all the late nights in the lab and extended weekends of studying. I could not have done this without you. 


\section{Table of Contents}

List of Tables $\quad$ vii

List of Figures $\quad$ viii

1 Introduction $\quad 1$

1.1 Motivation and Background . . . . . . . . . . . . . . . . 1

1.2 Thesis Objectives . . . . . . . . . . . . . . . . . . . . . . . 2

1.3 Thesis Outline . . . . . . . . . . . . . . . 3

2 Piezoelectric Materials 5

2.1 General Piezoelectric Overview . . . . . . . . . . . . . . . . 5

2.2 Hysteresis, Domain Switching and Energy Walls _ . . . . . . . . . . 10

3 Experiments $\quad 16$

3.1 Experimental Setup . . . . . . . . . . . . . . . . . . 16

3.1 .1 Test Specimen _ . . . . . . . . . . . . . . . . . 16

3.1.2 Strain Measurement . . . . . . . . . . . . . . . . 17

3.1.3 Polarization Measurement . . . . . . . . . . . . . . . . . . . 18

3.1.4 The Computer Control System . . . . . . . . . . . . . . . . 21

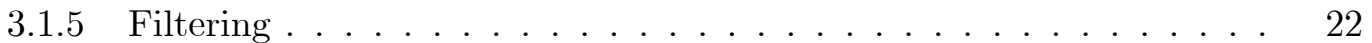

3.1 .6 Leakage Current . . . . . . . . . . . . . . . . . . . . . 30

3.2 Actuator Behavior under Pure Electrical Loading . . . . . . . . . . . . . . . 32

3.2 .1 Test Procedures _ . . . . . . . . . . . . . . . . . . 33

3.3 Full-Loop Behavior . . . . . . . . . . . . . . . . . . . . . . 37

3.4 Minor-Loop Behavior . . . . . . . . . . . . . . . . . . . . . 39

3.4.1 First Order Reversals . . . . . . . . . . . . . . . . . . . . . . 39

3.4 .2 Second Order Reversals . . . . . . . . . . . . . . . . . . . . 42

3.4.3 Third Order Reversals . . . . . . . . . . . . . . . . . . . 45

3.4.4 Fourth Order Reversals . . . . . . . . . . . . . . . . . 47

3.5 Creep Behavior . . . . . . . . . . . . . . . . . . . . . . . 48

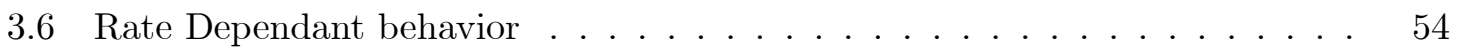

3.6 .1 Full-Loop . . . . . . . . . . . . . . . . . 54 
3.6.2 First Order Reversals . . . . . . . . . . . . . . . . . 57

3.6.3 Second Order Reversals . . . . . . . . . . . . . . . . . . . 60

3.6.4 Third Order Reversals . . . . . . . . . . . . . . . . . . . . 61

3.6.5 Fourth Order Reversals . . . . . . . . . . . . . . . . . 61

3.6.6 Leakage Current Effects on Hysteresis and Creep Curves . . . . . . . 62

3.7 Conclusions . . . . . . . . . . . . . . . . . . 65

4 Modeling of Energy $\quad 66$

4.1 Energy Model for Single Crystal PZTs . . . . . . . . . . . . . . 66

$\begin{array}{lll}5 & \text { Conclusions } & 76\end{array}$

$\begin{array}{ll}\text { List of References } & 78\end{array}$

A Simulink/Matlab Files $\quad \mathbf{8 2}$

A.1 Simulink Block Diagram . . . . . . . . . . . . . . . . 82 


\section{List of Tables}

3.1 Technical data for P802.00 PZT stack actuator . . . . . . . . . . . . . 17

3.2 Static noise reduction and delay for a low pass filter using different cut off frequencies. . . . . . . . . . . . . . . . . . 24

3.3 Static noise reduction and delay for averaging scheme filter using different sample numbers. . . . . . . . . . . . . . . . . . 25

3.4 Effective resolution and delay for different filters. . . . . . . . . . . 26

3.5 Range of cut off frequency for filters on different loading rates. . . . . . . . 30

3.6 Maximum loading rates for different voltage ranges. . . . . . . . . . . . 37 


\section{List of Figures}

2.1 Electric dipoles in Weiss domains; (1) unpoled ferroelectric ceramic, (2) during and (3) after poling (piezoelectric ceramics) . . . . . . . . 7

2.2 PZT unit lattice cell . . . . . . . . . . . . . . . . . . . . 8

2.3 Various orientations of a simple 2D single crystal unit lattice cell structure. $\quad 9$

2.4 Orientations of a simple 2D single crystal unit lattice cell for PZT as they correspond to a changing electric field. . . . . . . . . . . . . . . 10

2.5 Hysteresis curves of (a) polarization vs electric field and (b) strain vs. electric field for a single crystal along with the crystal lattice cell orientations. . . . 11

2.6 Hysteresis curves for single crystal showing only two phases. . . . . . . . . . 12

2.7 Single crystal hysteresis curve with experimental data. (a) Polarization vs. electric field and (b) Strain vs electric field. . . . . . . . . . . . . . 13

2.8 Single crystal model strain vs. electric field hysteresis curve with theoretical two phase polycrystalline hysteresis. . . . . . . . . . . . . . . 14

2.9 Single crystal hysteresis curves including $90^{\circ}$ phase. . . . . . . . . . . . . 14

2.10 Single crystal hysteresis curve with experimental data including $90^{\circ}$ phase. $\quad 15$

3.1 PZT Stage . . . . . . . . . . . . . . . . . . . . . . 18

3.2 Sawyer-Tower Circuit . . . . . . . . . . . . . . . . . . 19

3.3 Noise content of static signal from optical displacement sensor. . . . . . . . 22

3.4 Power spectrum of fast Fourier transform of static signal from optical sensor. 23

3.5 Unfiltered vs filtered plots of strain vs electric field for an electric loading rate of $.05 \mathrm{kV} / \mathrm{mm} / \mathrm{sec} \ldots \ldots \ldots \ldots \ldots 28 \ldots \ldots \ldots$

3.6 Unfiltered vs filtered plots of strain vs. electric field for an electric loading rate of $.5 \mathrm{kV} / \mathrm{mm} / \mathrm{sec} \ldots \ldots \ldots \ldots \ldots$

3.7 Unfiltered vs filtered plots of strain vs. electric field for an electric loading

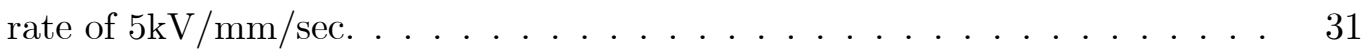

3.8 Leakage current rate as a function of the applied voltage to the actuator. . $\quad 33$

3.9 Polarization measurement error caused by leakage current for a typical long running experiment. . . . . . . . . . . . . . 34

3.10 Polarization vs. electric field hysteresis loop with cyclic linear electric loading between $\pm 1.5 \mathrm{kV} / \mathrm{mm}$. . . . . . . . . . . . . . . . . 35

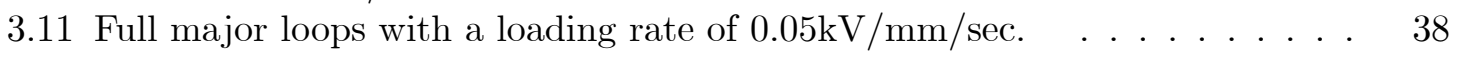


3.12 First order reversals, with a loading rate of $0.05 \mathrm{kV} / \mathrm{mm} / \mathrm{sec}$. . . . . . . .

3.13 First order reversals, polarization vs. electric field and strain vs. electric field with zooms into reversal points. Loading rate of $0.05 \mathrm{kV} / \mathrm{mm} / \mathrm{sec}$. . . . . . 41

3.14 Second order reversals, loading rate of $0.05 \mathrm{kV} / \mathrm{mm} / \mathrm{sec}$. . . . . . . . . 43

3.15 Second order reversals, polarization vs. electric field and strain vs. electric field with zooms into reversal points. Loading rate of $0.05 \mathrm{kV} / \mathrm{mm} / \mathrm{sec}$. . . . 44

3.16 Third order reversals, Loading rate of $0.05 \mathrm{kV} / \mathrm{mm} / \mathrm{sec} \ldots \ldots$. . . . . . . 46

3.17 Third order reversals, polarization vs. electric field and strain vs. electric field with zooms into reversal points. Loading rate of $0.05 \mathrm{kV} / \mathrm{mm} / \mathrm{sec}$. . . . 47

3.18 Fourth order reversals, loading rate of $0.05 \mathrm{kV} / \mathrm{mm} / \mathrm{sec} \ldots \ldots$. . . . . . 49

3.19 Fourth order reversals, polarization vs. electric field and strain vs. electric field with zooms into reversal points. Loading rate of $0.05 \mathrm{kV} / \mathrm{mm} / \mathrm{sec}$. . .

3.20 Polarization and strain vs time for observation of creep behavior. (a) The applied electric field. (b) Polarization vs. time. (c) Strain vs. time. . . . . . 51

3.21 Polarization vs. electric field hysteresis for creep experiment. . . . . . . . . 52

3.22 Strain vs. electric field hysteresis for creep experiment. . . . . . . . . . . 53

3.23 Full-loops, loading rates of $0.05 \mathrm{kV} / \mathrm{mm} / \mathrm{sec}, 0.5 \mathrm{kV} / \mathrm{mm} / \mathrm{sec}$, and $5 \mathrm{kV} / \mathrm{mm} / \mathrm{sec} .55$

3.24 Full-loop polarization vs. electric field hysteresis at different loading rates. . 56

3.25 Full-loop strain vs. electric field hysteresis at different loading rates. . . . . 56

3.26 First-order inner-loop hysteresis for different loading rates. (a-c) Polarizatin vs electric field. (d-f) Strain vs electric field. . . . . . . . . . . . . 58

3.27 First order inner-loop hysteresis curves for creep experiments at different loading rates. (a-c) Polarizatin vs electric field. (d-f) Strain vs electric field.

3.28 Second order reversal hysteresis curves at different loading rates. (a-c) Polarizatin vs electric field. (d-f) Strain vs electric field. . . . . . . . . . . . 60

3.29 Third order reversal hysteresis curves at different loading rates. (a-c) Polarizatin vs electric field. (d-f) Strain vs electric field. . . . . . . . . . . . . .

3.30 Fourth order reversal hysteresis curves for different loading rates. (a-c) Polarizatin vs electric field. (d-f) Strain vs electric field. . . . . . . . . . . . .

3.31 Leakage current effect on polarization hysteresis for third order reversal curves at a loading rate of $0.05 \mathrm{kV} / \mathrm{mm} / \mathrm{sec} \ldots \ldots \ldots$. . . . . . .

3.32 Leakage current effect on polarization creep behavior at a loading rate of $0.05 \mathrm{kV} / \mathrm{mm} / \mathrm{sec} \ldots \ldots \ldots \ldots \ldots$

4.1 Polarization vs electric field for experimental data fitted with a single crystal approximation curve. Dashed line is theoretical single crystal curve, while solid curve is experimental curve. . . . . . . . . . . . . . . 68

4.2 Helmholtz free energy as a function of polarization. . . . . . . . . . . . 69

4.3 Gibbs energy for a single crystal as a function of polarization with increasing electric field (a-d), and the corresponging single crystal polarization vs electric field $(\mathrm{e}-\mathrm{h}) . \ldots \ldots \ldots \ldots \ldots$ 
4.4 Simulation of switching behavior for full-loop hysteresis at a loading rate of

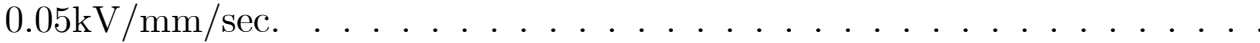

4.5 Simulation of switching behavior for full-loop hysteresis at a loading rate of

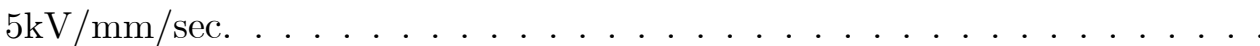

4.6 Simulation of switching behavior for first order reversals of inner-loop experiments at $0.05 \mathrm{kV} / \mathrm{mm} / \mathrm{sec} \ldots \ldots \ldots \ldots \ldots$. . . . . . . . . . . . 75

A.1 Simulink Block Diagram . . . . . . . . . . . . . . . . . . . 82 


\section{Chapter 1}

\section{Introduction}

\subsection{Motivation and Background}

Piezoceramic actuators are used in a large variety of applications. With the increased drive toward nanotechnology, the need for actuators that can perform nano-positioning has grown. One of the main complications with nano-positioning is the lack of actuators that can perform with nano-scale resolution. The use of piezoceramics has become the most promising solution to actuation with nano-scale resolution. Many piezoceramic devices such as stack actuators and translation stages are used in nano manufacturing, scanning tunneling microscopes (STM), atomic force microscopes (AFM), diagnostics or monitoring of biological processes, and many other sub-micron positioning applications [32], [34], [35], [36], [37], [38], [39], [40], [41].

Piezoceramic actuators are attractive due to their fast frequency response, high resolution, large force and high stiffness. However, inherent in piezoceramic material are many undesirable characteristics such as non-linearity, hysteresis, rate dependence, temperature dependence, and creep. In fact, many mathematical models of PZT behavior have been suggested in the literature [22] [24] [25], [13], [12] to describe the nonlinearity and hysteresis. The most well known model is the Preisach model [8] which Ping Ge and Musa Jouanah [13] used in conjunction with a feedback method to control a one dimensional piezoceramic actuator to improve tracking errors to as little as .02um to .45um. Many improvements and additions to Preisach's model have been used as well [10], [9], [12], [14]. A problem with 
Preisach-based models is that they are based on a mathematical concept without clear physical background, and as such do not account for rate-dependence or creep behavior without the addition of highly specified operators [12], [14] that only work for limited cases. These non-linear behaviors are known to be due to the kinetics of the domain switching process in the material which has need studied in [6], [7], [17], [18], [19], [29]. However, most of these models confine attention to the rate-independent limit of the material behavior, and only recently, Smith et al. [27], [26], [28], have proposed a free energy model based on the theory of thermal activation, which is capable of describing these effects in a natural way.

A literature survey revealed that most of the experimental work in the field has either been devoted to extremely high loading rates in the acoustic region [14] where no domain switching occurs, or they focus on full-loop behavior only [7],[19],[20], [29]. More recently, Zhang and Bhattacharya (2004) [30] were able to accurately simulate the nucleation of domain walls with full-loop experimental data but do not explore the inner-loop behavior where the $90^{\circ}$ phase transformations are more pronounced. It is, however, very important to study the inner-loop behavior for different rates in order to accurately identify model parameters.

To the best of our knowledge, this thesis gives a focused study on the domain switching and rate dependant behavior of the material through systematic experimental investigation of full-loop, minor-loop, and creep behavior for the first time.

\subsection{Thesis Objectives}

The objectives of this thesis is to produce systematic experiments that investigate the rate dependence and domain switching behavior of a typical piezoelectric actuator. It will also briefly introduce a energy based graphical model of the domain switching process. The experiments will focus on the hysteresis behavior for minor loops created by reversals within the full major loop. The thesis will be presented in four parts.

The first goal is to produce detailed documentation of the hysteretic behavior of the material under piecewise linear electrical loading. The electric field will be reversed at systematic locations in order to investigate switching behavior between $\pm 180^{\circ}$ and $90^{\circ}$ phase variant orientations. We are mostly interested in reversal behavior during the domain 
switching processes with particular attention on how the hysteresis change depending on which phase variant orientation is predominately present.

Second, using the same actuator, this thesis will produce detailed documentation on the rate effects on the actuators behavior. This set of experiments will use the same piecewise linear loading, and same reversal locations, as before with different loading rates. The changing hysteretic behavior is observed as the electrical linear loading rate is increased by two orders of magnitude.

Third, using the same actuator, this thesis will produce detailed documentation on the creep, or relaxation, behavior of the material. The material is held under varying constant electric fields for 15 seconds during which the relaxation behavior of the material is observed. The main focus of this set of experiments is to document the changing relaxation behavior of the material during different orientations of crystallite domains.

The fourth part of this thesis uses energy relations to illustrate the domain switching of the material to gain a better understanding on the behavior observed during the conducted experiments.

\subsection{Thesis Outline}

This thesis is divided into five chapters as follows:

Chapter 1 motivates the necessity of the research along with a literature review of the previous work on piezoelectric material. This chapter also outlines the objectives and structure of this thesis.

Chapter 2 focuses on an overview of what piezoelectric materials are and provides a basic understanding of its dynamics. The chapter will start with a basic piezoelectric overview followed by a simple model of a single crystal and its dynamics.

Chapter 3, the bulk of this thesis, focuses on the experimental investigation of the material. The chapter contains and overview of the experimental setup, data acquisition techniques and experimental procedures as well as a summarization of the results. The actuator is investigated under pure electrical loading only. The Strain and Polarization of the material are measured and plotted against time as well as the applied electric field to produce the hysteresis curves. There are three main interests in these experiments all of 
which have to do with the hysteretic behavior of the material while piecewise linear electric loads, with varying reversal points, are applied to the material. The first interest is in observing the material behavior while the electric field is reversed at those points. Another is to observe the material behavior as the electric field is held constant at those points. The last is to observe the materials behavior while the loading rate for the prescribed piecewise curve is increased by two orders of magnitude.

Chapter 4 introduces a free energy model that includes $90^{\circ}$ domain switching for the description of piezoelectric materials. The model will help illustrate the hysteretic behavior of the piezoceramics presented in the previous chapters. The model will produce the Helmholtz free energy as a function of polarization. To illustrate the dynamic behavior of the domain switching processes, the Gibbs free energy will be incorporated into the model and simulations run against experimental data.

Chapter 5 will present concluding remarks on the results and comment on future work. 


\section{Chapter 2}

\section{Piezoelectric Materials}

This chapter focuses on an overview of what piezoelectric materials are and provides a basic understanding of its dynamics. The chapter will start with a basic piezoelectric overview followed by a simple model of a single crystal and its dynamics.

\subsection{General Piezoelectric Overview}

The effect known as piezoelectricity was discovered by brothers Pierre and Jacques Curie when they were 21 and 24 years old in 1880. The word piezo is Greek for "push". Certain crystals are called piezoelectric when they exhibit a relationship between mechanical strain (tension or compression) and voltage across their surfaces. Specifically, when compressed or pulled, a piezoelectric crystal will build up alternate charges on opposite faces, thus acting like a capacitor with an applied voltage. A current, called piezoelectricity, can then be generated between the faces. On the other hand, when subjected to an external voltage, the crystal will expand or contract accordingly. For the experiments in this thesis, the piezoceramic will be subjected to various external voltages only.

Quartz demonstrates this piezoelectric property and is extremely stable. Quartz crystals are used for watch crystals and for precise frequency reference crystals for radio transmitters. Rochelle salt produces a comparatively large voltage upon compression and was used in early crystal microphones. Barium titanate, lead zirconate, and lead titanate are ceramic materials which exhibit piezoelectricity and are used in ultrasonic transducers as well as microphones. If an electrical oscillation is applied to such ceramic wafers, they will 
respond with mechanical vibrations which provide the ultrasonic sound source. The standard piezoelectric material for medical imaging processes has been lead zirconate titanate (PZT). Piezoelectric ceramic materials have found use in producing motions on the order of nanometers in the control of scanning tunneling microscopes.

Since the piezo effect exhibited by natural materials such as quartz, tourmaline, Rochelle salt, etc. is very small, polycrystalline ferroelectric ceramic materials such as BaTiO3 and PZT have been developed with improved properties. Ferroelectric ceramics become piezoelectric when poled. Piezo ceramics are available in many variations and are still the most widely used materials for actuator or sensor applications today. Piezo crystallites are centrosymmetric cubic (isotropic) before poling and after poling exhibit tetragonal symmetry (anisotropic structure) below the Curie temperature. Above this temperature they lose the piezoelectric properties.

Charge separation between the positive and negative ions is the reason for electric dipole behavior. Groups of dipoles with parallel orientation are called Weiss domains. The Weiss domains are randomly oriented in the raw Piezo material, before the poling treatment has been finished. For this purpose an electric field $(>2000 \mathrm{~V} / \mathrm{mm})$ is applied to the (heated) piezo ceramics. With the field applied, the material expands along the axis of the field and contracts perpendicular to that axis. The electric dipoles align and roughly stay in alignment upon cooling. The material now has a remanent polarization (which can be degraded by exceeding the mechanical, thermal and electrical limits of the material). As a result, there is a distortion that causes growth in the dimensions aligned with the field and a contraction along the axes normal to the electric field.

When an electric voltage is applied to a poled piezoelectric material, the Weiss domains increase their alignment proportional to the voltage. The result is a change of the dimensions (expansion, contraction) of the Piezo material.

Piezoelectric materials are characterized by several coefficients:

Examples are:

$\mathbf{d}_{i j}$ : Strain coefficients $[\mathrm{m} / \mathrm{V}]$ : strain developed $(\mathrm{m} / \mathrm{m})$ per electric field applied $(\mathrm{V} / \mathrm{m})$ 


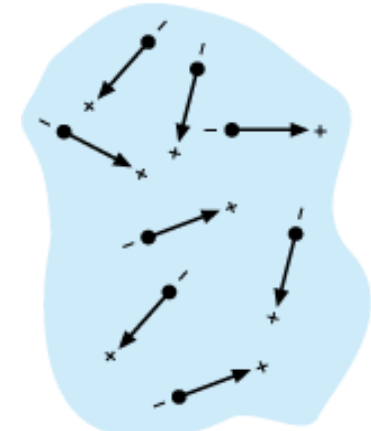

(1)

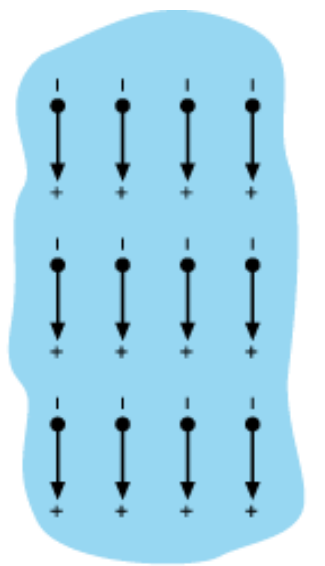

(2)

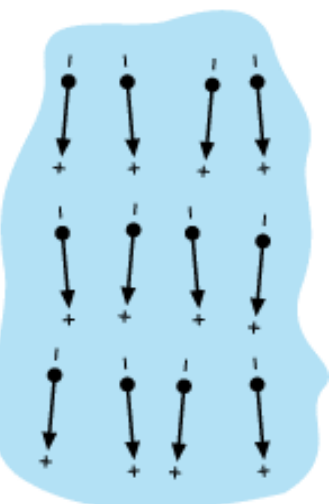

(3)

Figure 2.1: Electric dipoles in Weiss domains; (1) unpoled ferroelectric ceramic, (2) during and (3) after poling (piezoelectric ceramics)

or (due to the sensor / actuator properties of Piezo material).

Charge output coefficients $[\mathrm{C} / \mathrm{N}]$ : charge density developed $\left(\mathrm{C} / \mathrm{m}^{2}\right)$ per given stress $\left(\mathrm{N} / \mathrm{m}^{2}\right)$.

$\mathbf{g}_{i j}$ : Voltage coefficients or field output coefficients $[\mathrm{Vm} / \mathrm{N}]$ : open circuit electric field developed $(\mathrm{V} / \mathrm{m})$ per applied mechanical stress $\left(\mathrm{N} / \mathrm{m}^{2}\right)$

or (due to the sensor / actuator properties of Piezo material)

Strain developed $(\mathrm{m} / \mathrm{m})$ per applied charge density $\left(\mathrm{C} / \mathrm{m}^{2}\right) . \mathrm{k}_{i j}$ : Coupling coefficients [no dimensions].

The coefficients are energy ratios describing the conversion from mechanical to electrical energy or vice versa.

$\mathbf{k}^{2}$ is the ratio of energy stored (mechanical or electrical) to energy (mechanical or electrical) applied.

Other important parameters are the Young's modulus Y (describing the elastic properties of the material) and the relative dielectric coefficients (permittivity) $\varepsilon$ (describing the capacitance of the material).

To link electrical and mechanical quantities double subscripts (e.g. $\mathrm{d}_{i j}$ ) are introduced. 


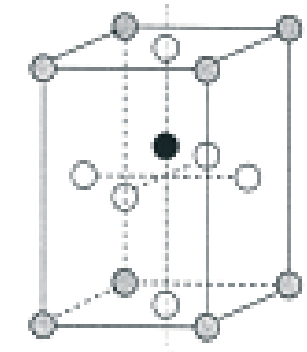

Positively Polarized $180 \mathrm{deg}$ crystal lattice cell

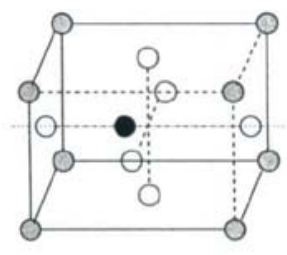

90deg crystal lattice cell

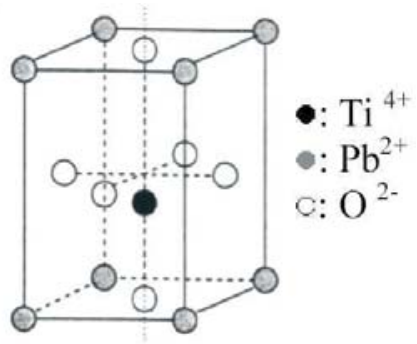

Negatively Polarized $180 \mathrm{deg}$ crystal lattice cell

Figure 2.2: PZT unit lattice cell

The first subscript gives the direction of the excitation, the second describes the direction of the system response.

Example:

$\mathrm{d}_{33}$ applies when the electric field is along the polarization axis (direction 3 ) and the strain (deflection) is along the same axis. $d_{31}$ applies if the electric field is in the same direction as before, but the strain is in the 1 axis (orthogonal to the polarization axis). In the experiments for this thesis, only $\mathrm{d}_{33}$ PZT effects are investigated.

A simple model of a unit lattice cell of a single crystal of PZT is shown in Figure 2.2. The alignment of the center titanium ion, black, is what determines the polarization. For the $\mathrm{d}_{33}$ orientation, the charge will build up on the top and bottom of the crystal depending on the center ions position. If the center ion is positioned in the $90^{\circ}$ phase, then no charge should accumulate in the $\mathrm{d}_{33}$ direction. The strain value, due to stretch or compression, will be the same if the center ion is negatively or positively polarized in the $180 \mathrm{deg}$. phase. The strain will be at its lowest value, for the $\mathrm{d}_{33}$ orientation, when the center ion is polarized in the 90 deg. phase. To better illustrate a single crystals form, a 2 dimensional model is shown in Figure 2.3.

In Figure 2.3, a $2 \mathrm{~d}$ unit lattice structure is shown for a single PZT crystal with various orientations of the center ion. (1), (2), and (3) correspond to a $+180^{\circ}$ orientation, while (5), (6) and (7) correspond to $-180^{\circ}$ orientation. notice that (1) and (7) have the same height and therefor will correspond to the same strain value even though the center ion 


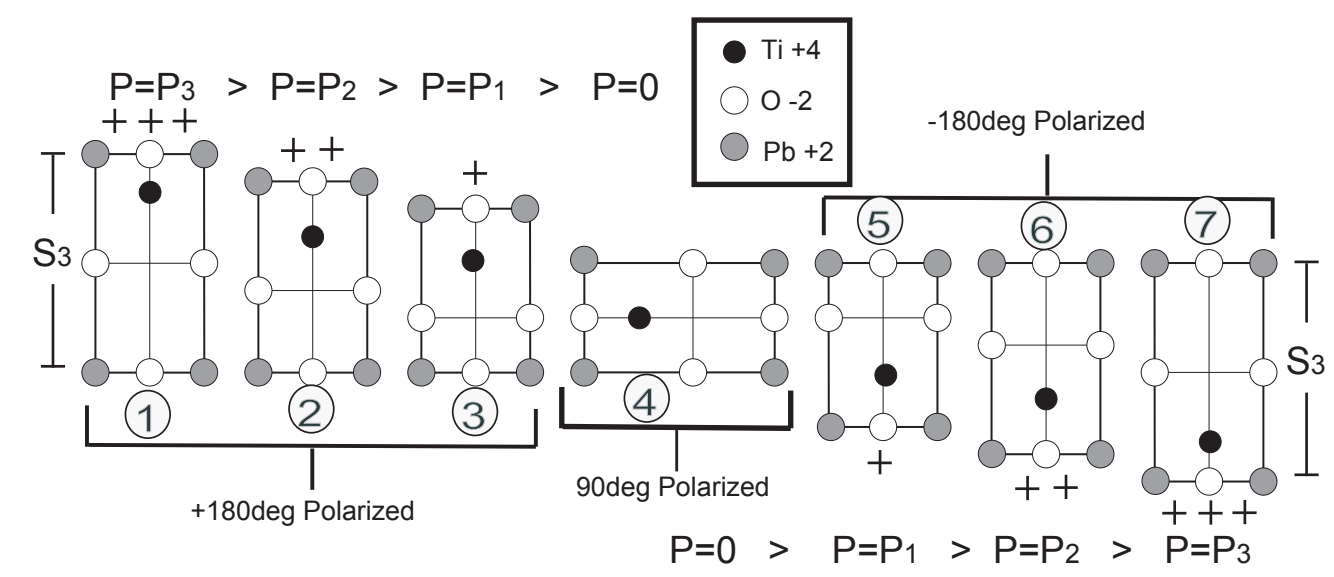

Figure 2.3: Various orientations of a simple 2D single crystal unit lattice cell structure.

is at an opposite polarity. The polarization magnitudes in (1) and (7) are also the same but opposite in sign. This symmetry is clearly shown for (2) and (6) as well as for (3) and (5). For the $+180^{\circ}$ orientation, (2) is an equilibrium position while (1) and (3) are the maximum and minimum ion positions while still maintaining a $+180^{\circ}$ phase. The center ion under an electric field will move out of the equilibrium position in (2) toward (1), with a applied positive electric field, or toward (3) with an applied negative electric field. If an applied negative electric field is strong enough, the ion will jump out of the $+180^{\circ}$ orientation and into the $90^{\circ}$ orientation which is also an unstable position (in the absence of any mechanical load) thus causing the ion to continue toward the $-180^{\circ}$ orientation. With the strong negative electric field, the ion will move quickly through orientations (5) and (6) toward orientation (7). If the electric field is then removed, the ion falls into a $-180^{\circ}$ equilibrium orientation at (6) which is symmetric to the $+180^{\circ}$ equilibrium orientation at (2). This process creates a repoling of the crystal. The new equilibrium position is at (6) rather than (2) where we started. The crystal has the same strain value since the $+180^{\circ}$ and $-180^{\circ}$ polarization forms are symmetric. This switching process for a single crystal subjected to purely electrical loading is shown in Figure 2.4.

In Figure 2.4 the single crystal starts with a $+180^{\circ}$ phase orientation which is an equilibrium position when the electric field equals zero. This electric loading process creates 


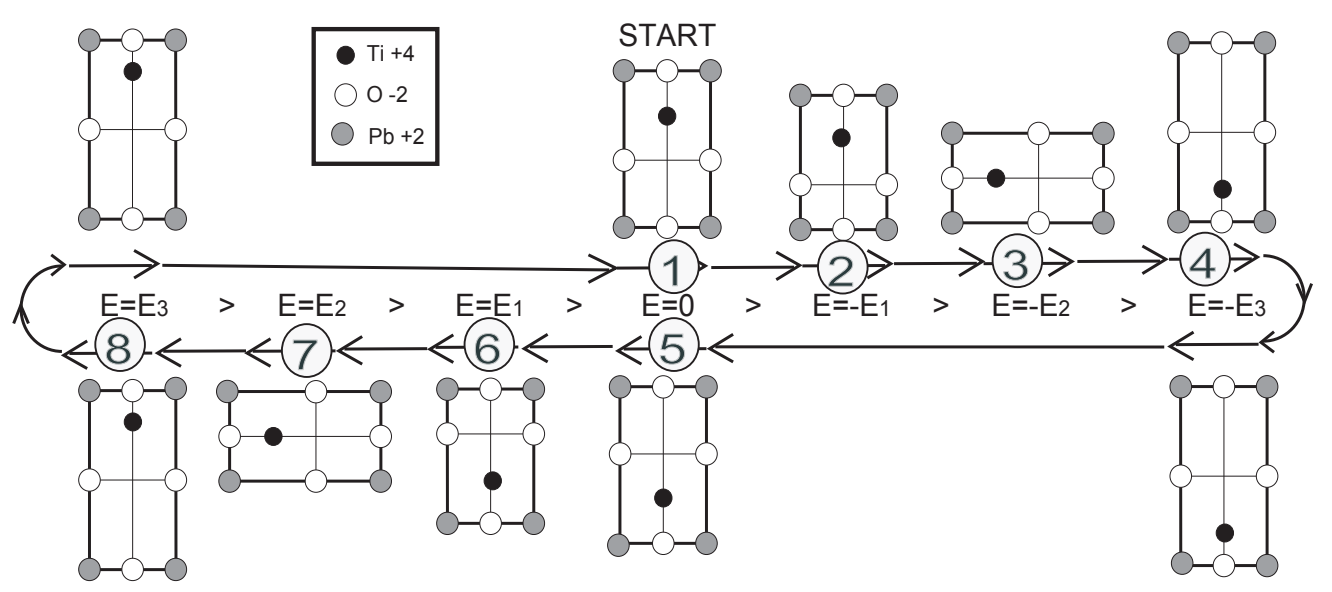

Figure 2.4: Orientations of a simple 2D single crystal unit lattice cell for PZT as they correspond to a changing electric field.

the hysteresis curves for Polarization vs. Electric Field and Strain vs Electric field shown in Figure 2.5. These hysteresis curves will be further explained in the next section.

\subsection{Hysteresis, Domain Switching and Energy Walls}

In order to simplify the materials dynamics, we will initially model only a single crystal. The hysteresis curves for single crystal are fairly simple and consist of a series of straight lines.

Early studies on PZT modeled the domain switching as a two step process. The center ion was thought to have only two polarities, poled positively $180^{\circ}$ or negatively $180^{\circ}$ and under electrical loading the ion would jump from one orientation to the other fairly instantly. More recent studies have shown that domains do not undergo $180^{\circ}$ switching directly, but rather an $180^{\circ}$ reorientation undergoes two successive $90^{\circ}$ switchings [6]. Using single crystal models and experimental data, it can be shown that indeed two successive $90^{\circ}$ switchings do occur, and thus the $90^{\circ}$ phase variance must be included in any modeling efforts.

First, the single crystal that does not include a $90^{\circ}$ phase variance will be explored. Figure 2.6 shows a typical single crystal hysteresis curves consisting of only 2 domain phase 
(a)
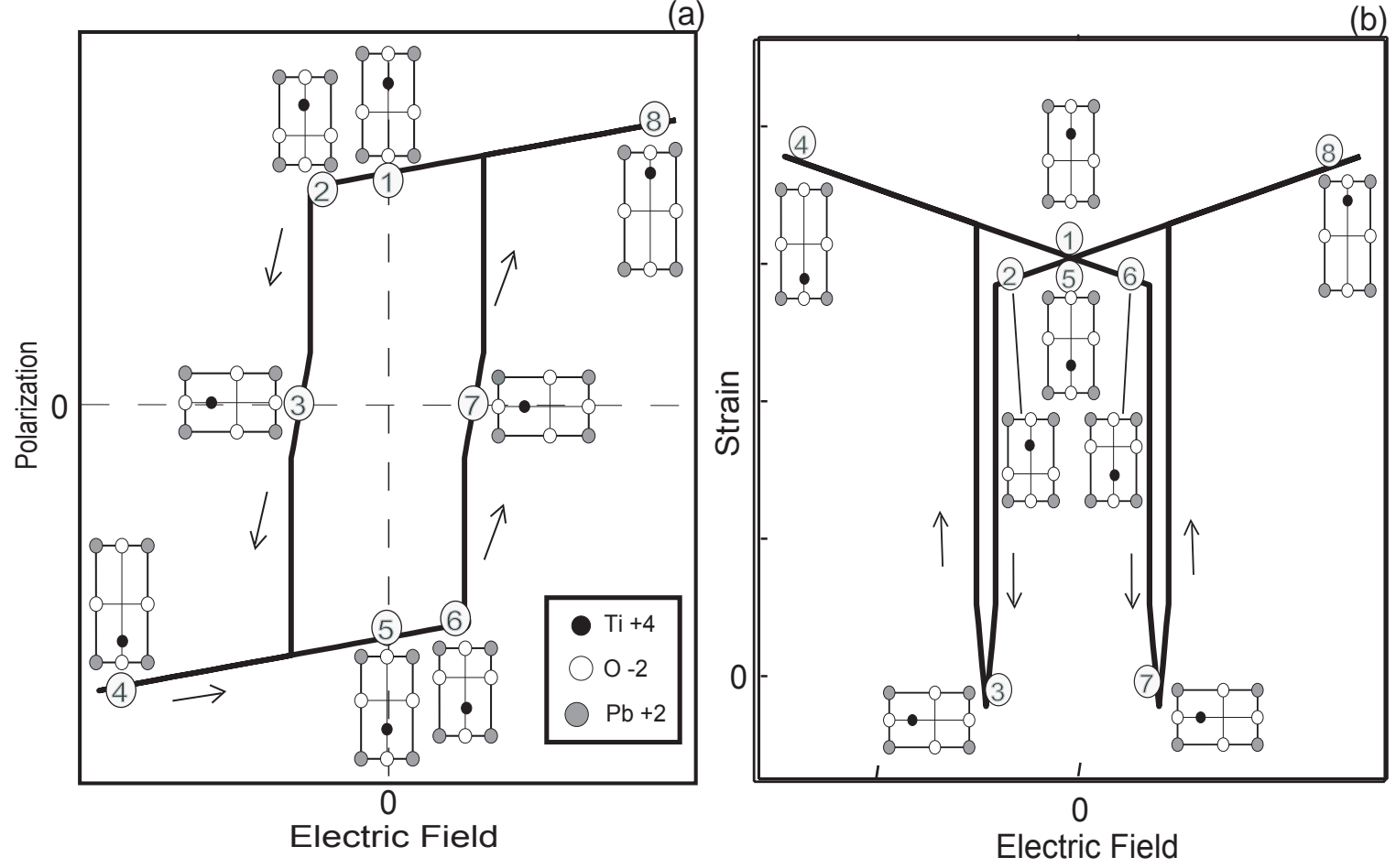

Figure 2.5: Hysteresis curves of (a) polarization vs electric field and (b) strain vs. electric field for a single crystal along with the crystal lattice cell orientations. 
variants. The numbers and small arrows indicate the flow path of the curve. The prescribed electric field starts at $1.5 \mathrm{kV} / \mathrm{mm}$ and is then brought to $-1.5 \mathrm{kV} / \mathrm{mm}$ at a constant linear rate. It is then returned to $1.5 \mathrm{kV} / \mathrm{mm}$ at the same rate.
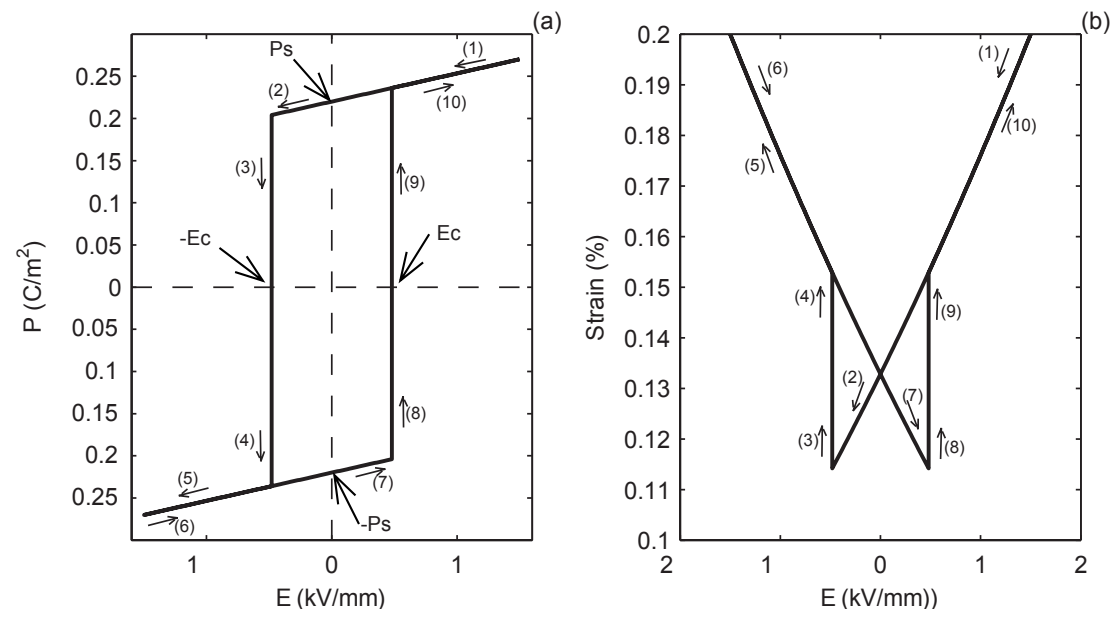

Figure 2.6: Hysteresis curves for single crystal showing only two phases.

In Figure 2.6 remanent polarization, $P_{s}$, is approximated from experimental data, this would be the polarization value when there is no applied electric field $(\mathrm{E}=0)$. The $E_{c}$ value is the value of the electric field when repoling occurs which is also approximated from experimental data. The slope of the curve before switching occurs (top and bottom lines will have the same slope) is also approximated from experimental data. This slope exists because the crystal will undergo very small electrostriction, that is to say that the center ion, while oriented in either $\pm 180^{\circ}$, will move along the $\mathrm{d}_{33}$-axis linearly with an applied electric field. This will create a very small linearly changing polarization and strain. The strain curve is found directly from the polarization curve. Through energy relations the strain is proportional to the polarization squared, that is: Strain $=\alpha P^{2}$. The value of $\alpha$ is approximated from experimental data. The curves in Figure 2.6 were created using our experimental data. Figure 2.7 shows the experimental data together with the single crystal hysteresis curves.

From the Figure 2.7 subplot (a) we can see that the experimental curve deviates form the single crystal curve. This is because the actual specimen consists of many crystals 

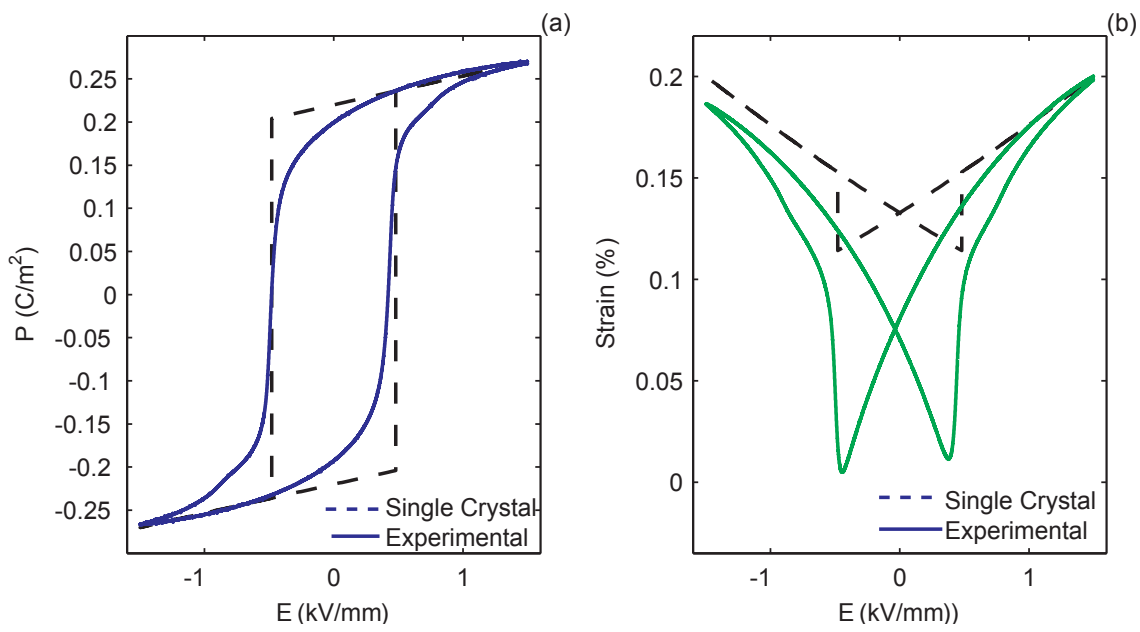

Figure 2.7: Single crystal hysteresis curve with experimental data. (a) Polarization vs. electric field and (b) Strain vs electric field.

with many different orientations and some of the crystals will switch variants before the critical $E_{c}$ value of the electric field is reached. When looking only at the polarization vs. electric field curve in Figure 2.7 subplot (a), there is no clear evidence of a $90^{\circ}$ phase. It is not until we look at the strain vs electric field curve in subplot (b) that the $90^{\circ}$ phase is shown. If we consider the specimen to be made up of many crystals and that, as found for the polarization, some of these crystals will switch phases early, then the experimental strain curve should deviate from the single crystal in an upward fashion back towards the maximum strain value as shown in Figure 2.8.

The experimental data for Strain vs electric Field in Figure 2.7 subfigure (b) does not show this. Instead, it shows the strain value deviating below the single crystal curve toward a minimum that could not be reached in the single crystal model with only 2 phases. The deviation downward toward the new minimum indicates the existence of a third phase, a $90^{\circ}$ phase, that could not be seen in the polarization full-loop hysteresis curve.

A newer single crystal model needs to be presented that accounts for the $90^{\circ}$ phase. Figure 2.9 shows single crystal hysteresis curves that includes a $90^{\circ}$ phase.

When we compare this model with the experimental data, shown in Figure 2.10, the curves behave more as expected. The experimental strain deviates below the single crystal 


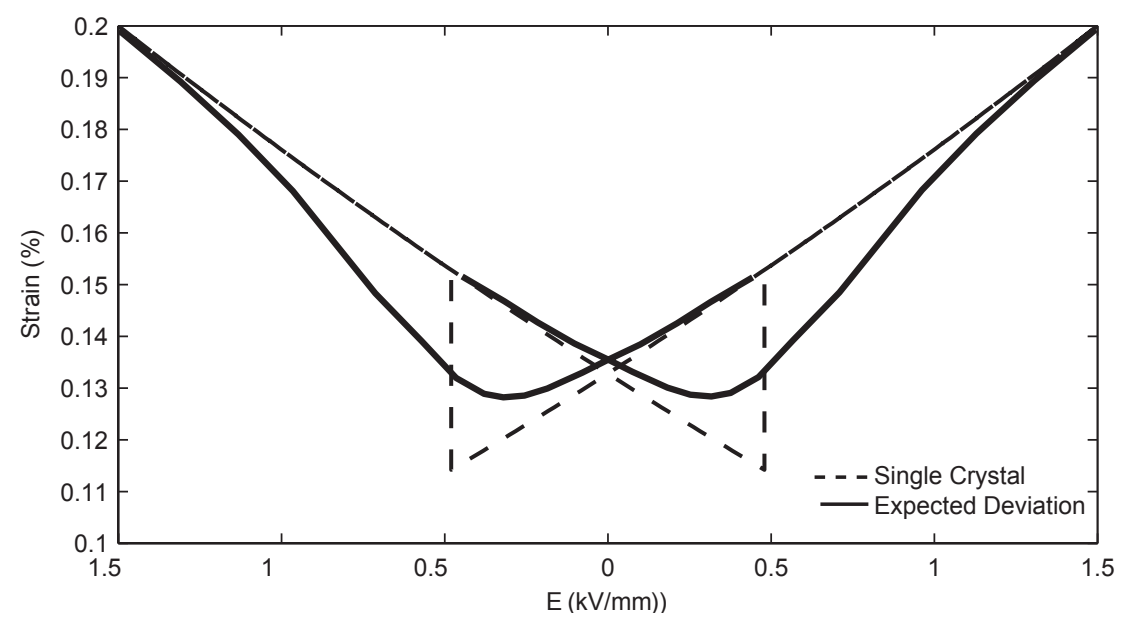

Figure 2.8: Single crystal model strain vs. electric field hysteresis curve with theoretical two phase polycrystalline hysteresis.
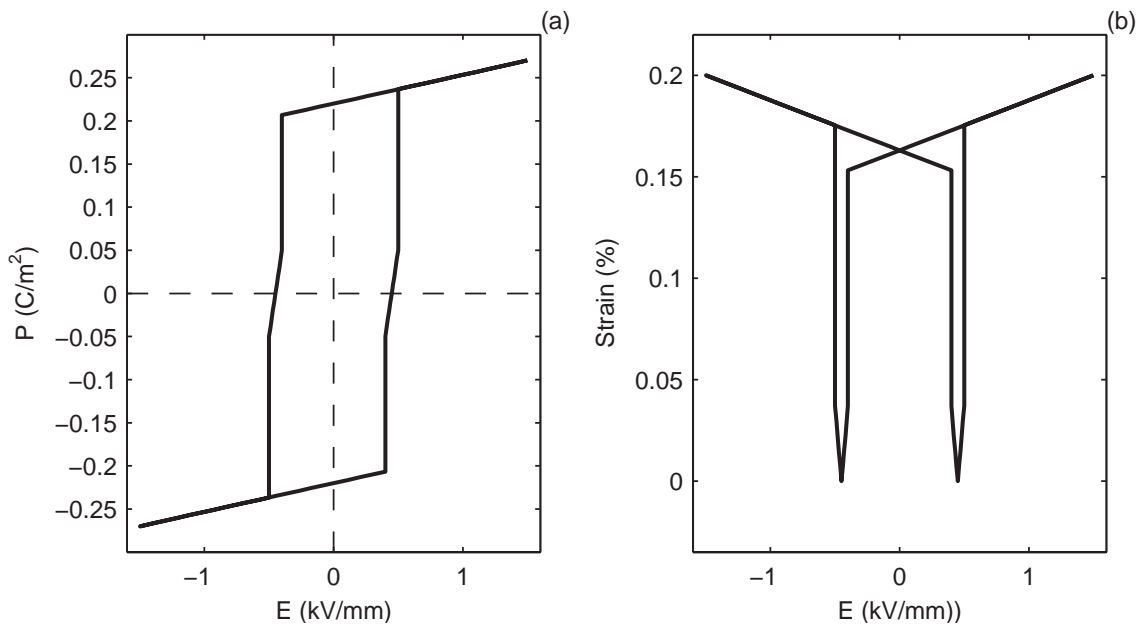

Figure 2.9: Single crystal hysteresis curves including $90^{\circ}$ phase. 

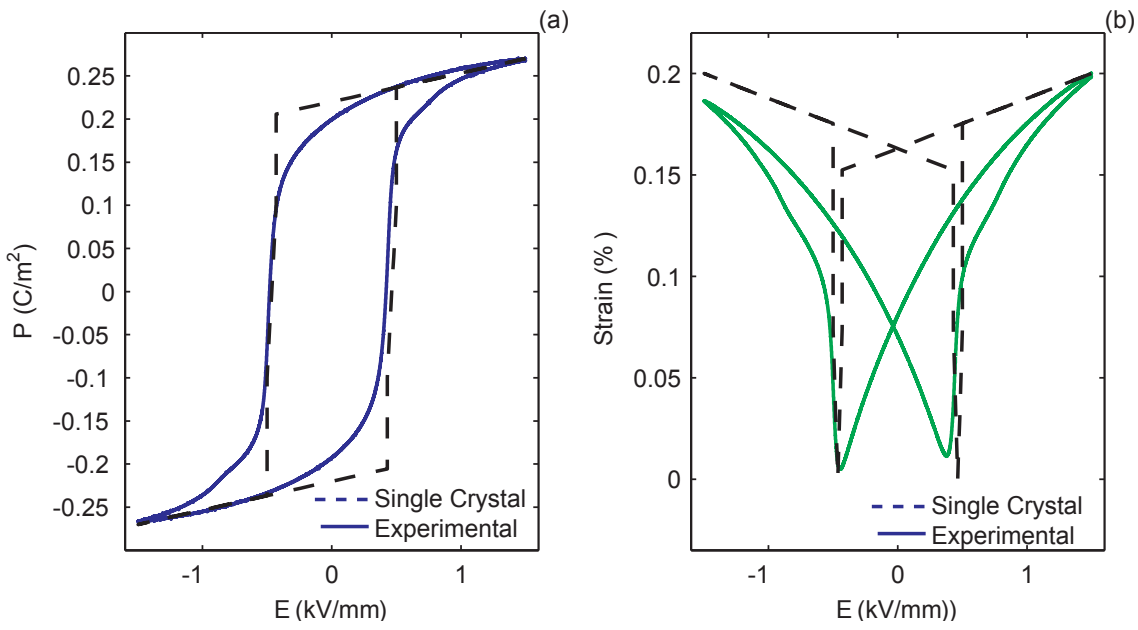

Figure 2.10: Single crystal hysteresis curve with experimental data including $90^{\circ}$ phase.

model due to the early switching into the $90^{\circ}$ phase of some of the crystals in the actual specimen. Likewise, there is some delayed switching from the $90^{\circ}$ phase to the $-180^{\circ}$ phase for the same reasons.

This thesis will focus on investigating the switching process for these three phases. The illustrative model introduced in chapter 4 will be based on the three phase variant orientations discussed in this chapter. 


\section{Chapter 3}

\section{Experiments}

This chapter investigates the experimental hysteretic behavior of a piezoceramic stack actuator. It contains an overview of the experimental setup, data acquisition techniques and experimental procedures as well as a summarization of the results. The actuator is investigated under pure electrical loading only. Piecewise linear loads are applied to the material with varying reversal points, and varying loading rates.

\subsection{Experimental Setup}

\subsubsection{Test Specimen}

The actuator chosen for the experiments is a commercially available PZT stack actuator from Physik Instrumente (www.pi.ws), Model P-802.00. This model has the best open-loop resolution in the product line and lowest current requirement, allowing easier setup and measurement. The actuator is made of the PIC151 soft PZT ceramic material proprietary to the PI Ceramics Company. The time effects of bulk PIC151 materials under electrical loading have been detailed in [2] and [3]. Previous research on this material provides us with basic information on the properties of the material and helps us verify our own experimental results, but the experiments in [3] are focused on the behavior of the material under constant electric fields and full cyclic electric fields under quasistatic conditions, while we are more interested in the material behavior of minor-loops from varying reversal points of the electric field and under varying loading rates. Important technical data of the actuator is listed in Table 3.1. 
Table 3.1: Technical data for P802.00 PZT stack actuator

\begin{tabular}{|c|c|}
\hline Open-loop travel @ 0 to 100V & $6 \mu \mathrm{m} \pm 20 \%$ \\
\hline Open-loop resolution & $0.06 \mathrm{~nm}$ \\
\hline Push/pull force capacity & $1000 / 5 \mathrm{~N}$ \\
\hline Electrical capacitance & $0.7 \mu \mathrm{F} \pm 20 \%$ \\
\hline Dynamic operating current coefficient(DOCC) & $15 \mu \mathrm{A} /(\mathrm{Hz} \times \mu \mathrm{m})$ \\
\hline Unloaded resonant frequency $\left(f_{0}\right)$ & $55 \mathrm{kHz} \pm 20 \%$ \\
\hline Standard operating voltage range & 0 to $100 \mathrm{~V}$ \\
\hline Standard operating temperature range & -20 to $80{ }^{\circ} \mathrm{C}$ \\
\hline Dimensions(with heatshrink packaging) & $7 \times 7 \times 9 \mathrm{~mm}$ \\
\hline Dimensions(without packaging) & $5 \times 5 \times 9 \mathrm{~mm}$ \\
\hline Layer thickness & $0.1 \mathrm{~mm}$ \\
\hline Number of active layers & 80 \\
\hline Equivalent surface area & $2000 \mathrm{~mm}^{2}$ \\
\hline
\end{tabular}

The actuator comes with epoxy insulation and heat shrink tube for protection and strain relief of the wire leads to facilitate mounting and driving. The standard operating voltage range of the actuator is defined in a range where non-linearity is less obvious, and the actuator has been prepoled to full positive polarization in the manufacturing process. With the goal to understand and model the hysteresis, the actuator was driven with a much wider voltage range. In practice, we found the actuator to work properly with at least $\pm 150 \mathrm{~V}$, which converts to an electric field of $\pm 1.5 \mathrm{kV} / \mathrm{mm}$. The material itself can survive an electric field of up to $5 \mathrm{kV} / \mathrm{mm}[3]$ but the stack layer packaging of the actuator is not rated for such high voltage ranges. We have found one of the specimens to break down after being driven at $200 \mathrm{~V}(2 \mathrm{kV} / \mathrm{mm})$ for an extended period of time. The displacement response of the broken actuator is almost unaffected, but the leakage current is significantly higher than normal. Therefore we limited our experiments to a range of $\pm 150 \mathrm{~V}( \pm 1.5 \mathrm{kV} / \mathrm{mm})$. The amplifier for driving the actuator (Model PZD350 Dual Channel Piezo Driver, Trek, Inc., Medina, NY) is capable of outputting $\pm 350 \mathrm{~V}$ at a slew rate greater than $500 \mathrm{~V} / \mu$ s with a current limit of $200 \mathrm{~mA}$.

\subsubsection{Strain Measurement}

To measure the strain of the actuator, a fiber-optic displacement sensor (Philtec RC12, Philtec, Inc. Annapolis, Maryland U.S.A.) is used to measure the displacement at the front 
surface of the actuator. The sensor has a standard resolution of 0.075 micron from DC$20 \mathrm{kHz}$. Accounting for the additional noise in our connection, we measured the sensor signal to have an effective resolution of 0.1 micron. The filtering process will be discussed in detail later in this chapter. The strain of the actuator is calculated by dividing the measured displacement by the total thickness of active layers. At $150 \mathrm{~V}(1.5 \mathrm{kV} / \mathrm{mm})$, the actuator has an extension of 13.3 microns, which converts to a strain of $13.3 /(100 \times 80) \times 100 \%=$ $0.16625 \%$. Figure 3.1 shows a picture of the mounted actuator together with the tip of the fiber-optic sensor. The actuator is glued to the mounting cap with epoxy, and its front surface is covered with a reflective tape to satisfy the optical requirement of the fiber-optic sensor.

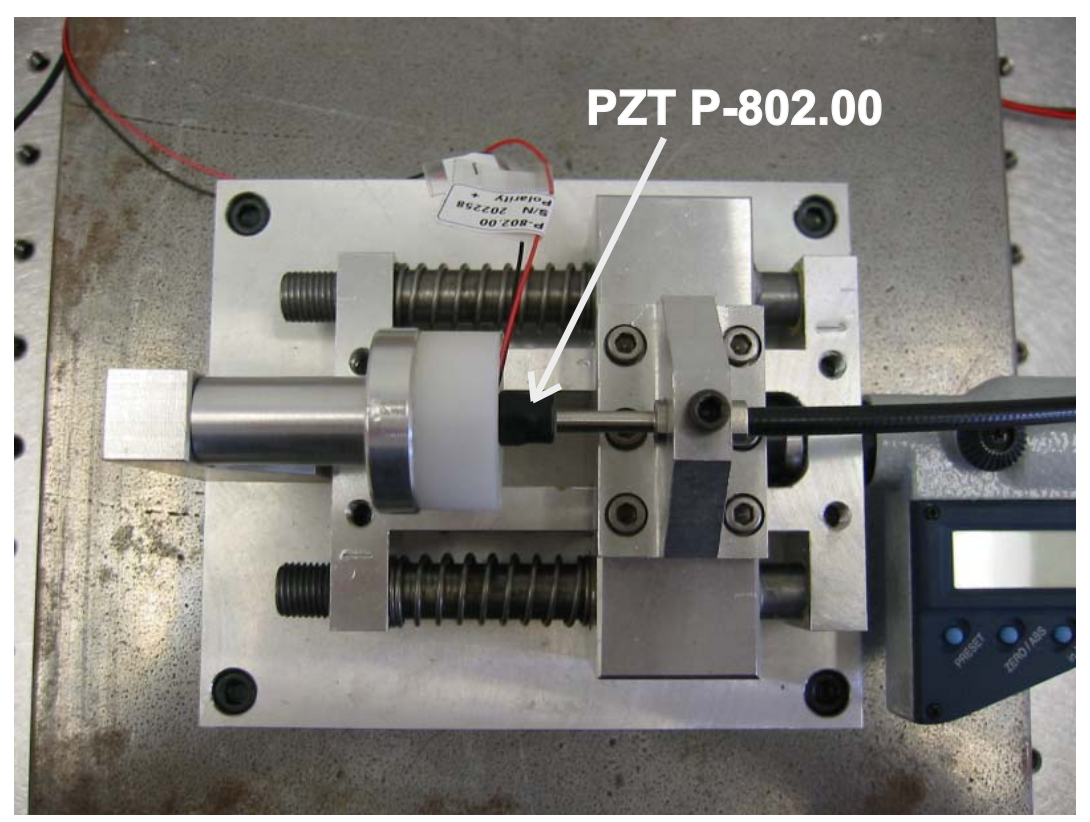

Figure 3.1: PZT Stage

\subsubsection{Polarization Measurement}

The polarization in piezoceramic materials under electrical loading is most commonly measured using the Sawyer-Tower method [4] [3], which is also what we use in our experiments. The schematic drawing of the test circuit is shown in Figure 3.2. 


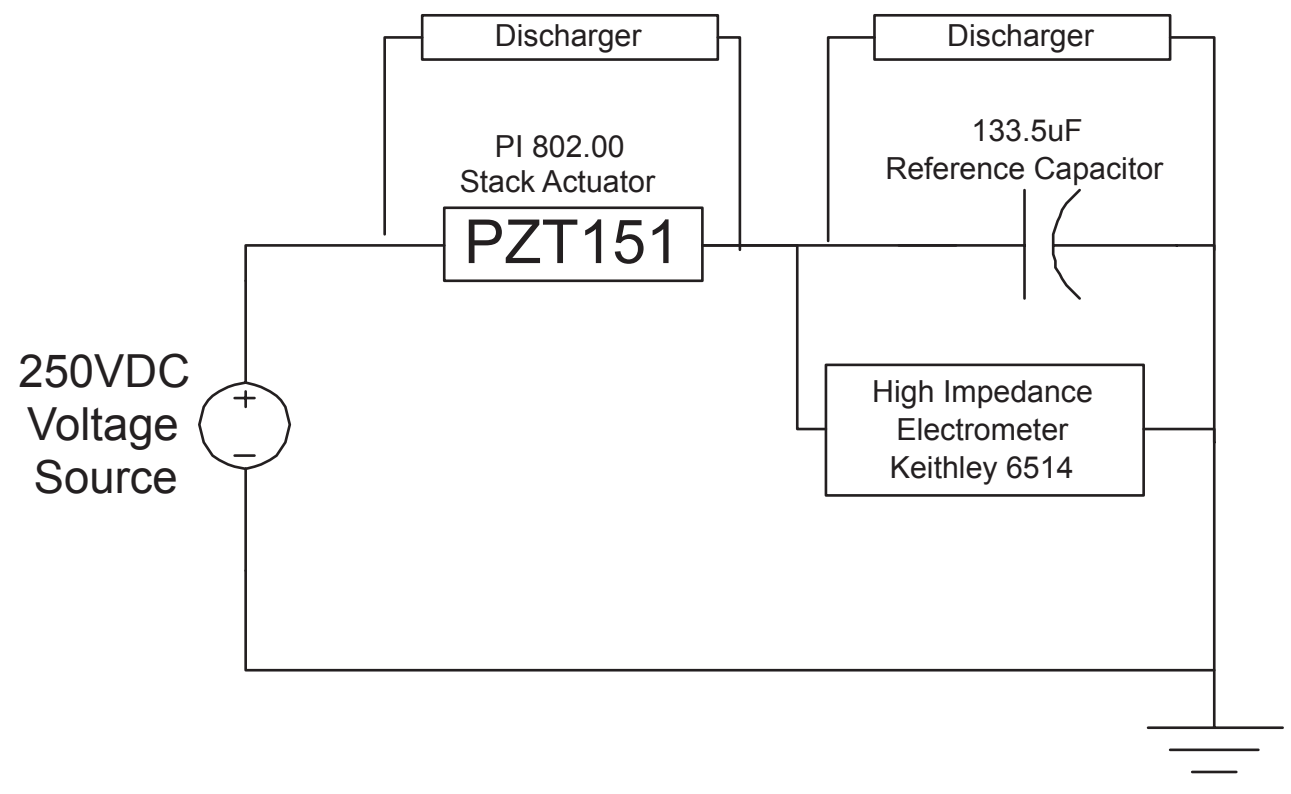

Figure 3.2: Sawyer-Tower Circuit

The key to the Sawyer-Tower method is the use of a reference capacitor connected in series with the PZT actuator, as shown in Figure 3.2. Polarization is defined as the dipole moment per unit volume or alternatively, the amount of charge per unit area. The charge on the PZT can not be measured directly, but the reference capacitor always takes the same amount of charge as the PZT because they are connected in series. The charge on the reference capacitor is

$$
Q_{r}=U_{r} C_{r}
$$

where $U_{r}$ is the voltage across the reference capacitor and $C_{r}$ is the capacitance of the reference capacitor. The polarization of the PZT actuator can then be calculated as

$$
P_{p z t}=\frac{Q_{r}}{A_{p z t}}=\frac{U_{r} C_{r}}{A_{p z t}}
$$

where $A_{p z t}$ is the equivalent area of the stack actuator. For the P802.00 actuator, $A_{p z t}=$ $2000 \mathrm{~mm}^{2}$. In the experiments, the voltage on the reference capacitor is first fed to a Keithley 6514 electrometer, which acts as a high impedance buffer to improve measurement quality. The voltage is then monitored and collected by the AD acquisition card in the computer control system, which is detailed in the next section. 
The choice of capacitance for the reference capacitor plays an important role in the measurement and control of the experiments. In principle, the capacitance of the reference capacitor should be as large as possible, so that its voltage $U_{r}$ is negligible compared to the voltage on the PZT and the voltage output from the amplifier $U_{a m p}$ acts almost entirely on the PZT. This also makes it easier for the AD system to measure the voltage on the reference capacitor, eliminating additional signal conditioning for high voltages outside the range of the $\mathrm{AD}$ board. To call the reference voltage $U_{r}$ negligible, the reference capacitance $C_{r}$ must be at least 1000 times larger than the PZT actuator capacitance $C_{p z t}$. However, the capacitance of the P802 PZT actuator is at least $0.8 \mathrm{uF}^{1}$, making it almost impossible to find a reference capacitor with a capacitance three orders of magnitudes large.

In our setup, we use 4 high-quality low-leakage polypropylene capacitors (Model X300, ASC Capacitors, Ogallala, NE) in parallel to get a reference capacitor of $C_{r}=133.5 \mathrm{uF}$. $C_{r}$ is large enough to bring the reference voltage $U_{r}$ into a desirable range of the AD acquisition system, but $U_{r}$ can still be as high as $6 \mathrm{~V}$ when the amplifier output $U_{a m p}$ is equal to $150 \mathrm{~V}$. In this case, the voltage on the PZT is only $U_{p z t}=U_{a m p}-U_{r}=150-6=144 \mathrm{~V}$. This is an error of $4 \%$ if we do not account for the voltage drop on the reference capacitor and consider $U_{p z t}$ to be equal to the amplifier output $U_{a m p}$. Therefore, we do not neglect the reference voltage when applying the voltage to the PZT. The voltage on the reference capacitor is measured and added to the prescribed PZT voltage in the control computer in real-time and the sum is used as the control signal to the amplifier. When the sampling frequency of the control system is high enough, the error of the PZT voltage due to the voltage drop on the reference capacitor will be compensated. This way we guarantee that the voltage on the PZT is indeed the voltage that we prescribe. Our computer control system samples at a maximum of $20 \mathrm{kHz}$ and the voltage error on the PZT is maintained below $0.5 \%$.

Another requirement in the measurement of polarization is the task of properly discharging the PZT and the reference capacitors after each experiment, otherwise the charge on the reference capacitor will keep accumulating until it overflows the $\mathrm{AD} / \mathrm{DA}$ system. For this reason, two discharger circuits using magnetic relays are added to the system, which are also controlled by the computer. When the experiments are finished and the discharger circuits

\footnotetext{
${ }^{1}$ This is the capacitance in the normal operating range. In the repoling process, the dynamic equivalent capacitance of the PZT becomes much larger.
} 
are activated, the PZT actuator and the reference capacitor will be separately discharged with a $10 \mathrm{k} \Omega$ resistor.

\subsubsection{The Computer Control System}

The computer control system for the experiments consists of two computers: One host computer for prescribing the test process, and one target computer dedicated to the execution of the experiments. The experiments are conducted using MATLAB 7.0/Simulink 5.0 with xPC Target 2.0. Simulink models for the experiments are first compiled into xPC Target programs in MATLAB/Simulink on the host PC running the Windows2000 operating system. Subsequently, the target programs are transferred to the target PC, the only task of which is to run the target program in a special real-time operating system environment. The performance of the host $\mathrm{PC}$ has no influence on the performance of the program running on the target PC. The target PC features an Athlon XP 1.6GHz CPU with 1GB of memory and runs the xPC Target 2.0 Real-time Operating System. For the Simulink applications used in our experiments, the target computer is capable of executing the model equations

and storing outputs in RAM within a time step of 0.00005 second, which is equal to a sampling frequency of $20 \mathrm{kHz}$.

After compiling and downloading the model application to the target computer, additional control on the prescribed test voltages is available from the host computer using a Matlab script file that updates the model parameters on the target computer. The collected experimental data is sent back to the host computer after each experiment for post processing and storage. The simulink model for the experiments are documented and included in the appendices of the thesis.

The control voltage to the amplifier and discharger, the voltage signal from the reference capacitor, the voltage and current monitor signal from the amplifier, and the voltage signal from the fiber-optic displacement sensor are all sent or received through one multi-channel AD/DA acquisition board (NI 6052E, National Instruments Corporation, Austin, TX) in the target computer. 


\subsubsection{Filtering}

Inherent in any data acquisition system is noise. We found the optical sensor signal to have a high amount of noise, while the polarization measurement signal had fairly low level noise due to the buffering from the electrometer. To remove the noise from our displacement signal, we tested two filters. The first filter is a simple transfer function filter found in the MATLAB 7.0/Simulink 5.0 library that acts as a low pass filter. The second filter is an adapting averaging scheme that we designed that also acts as a low pass filter by averaging over several samples.

Taking a static signal from the optical sensor, we can see that there is a high noise content. For a one second time duration, .00005 sec. sample time, and 300um distance from sensor to target, a peak-to-peak noise value of .003693 V, shown in Figure 3.3, that, with a sensitivity of $.0173 \mathrm{~V} / \mathrm{um}$ found in calibration, results in an effective resolution of $.2135 \mathrm{um}$.

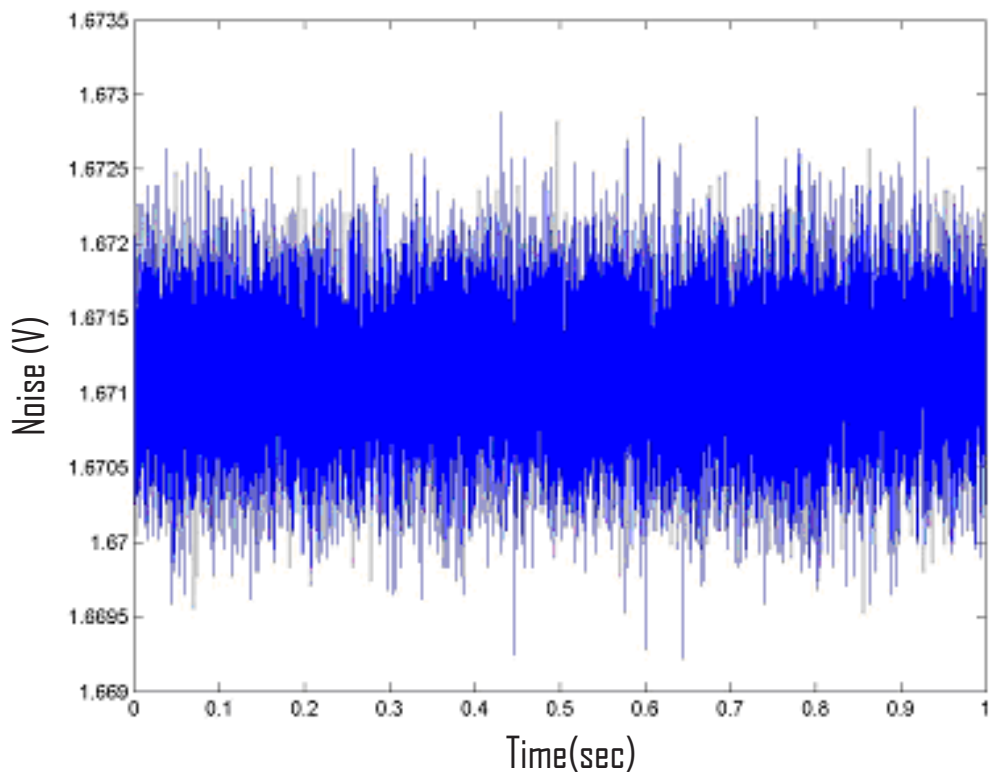

Figure 3.3: Noise content of static signal from optical displacement sensor.

The main costs associated with filtering are delay time and frequency limitations. These 
two costs have an inverse relationship; reducing one will cause an increase in the other. Delays will produce artificial phenomenon in the experimental data, which will be our main concern. The frequency limitations will limit the range of the loading rates for the experiments, but this will have to be sacrificed in order to be sure the data being produced is real and accurate. The first step in designing the best filter is to analyze the frequency content of the noise. A power spectrum plot of the FFT of the static noise signal, Figure 3.4, shows that most of the noise is fairly high frequency, above $1000 \mathrm{~Hz}$.

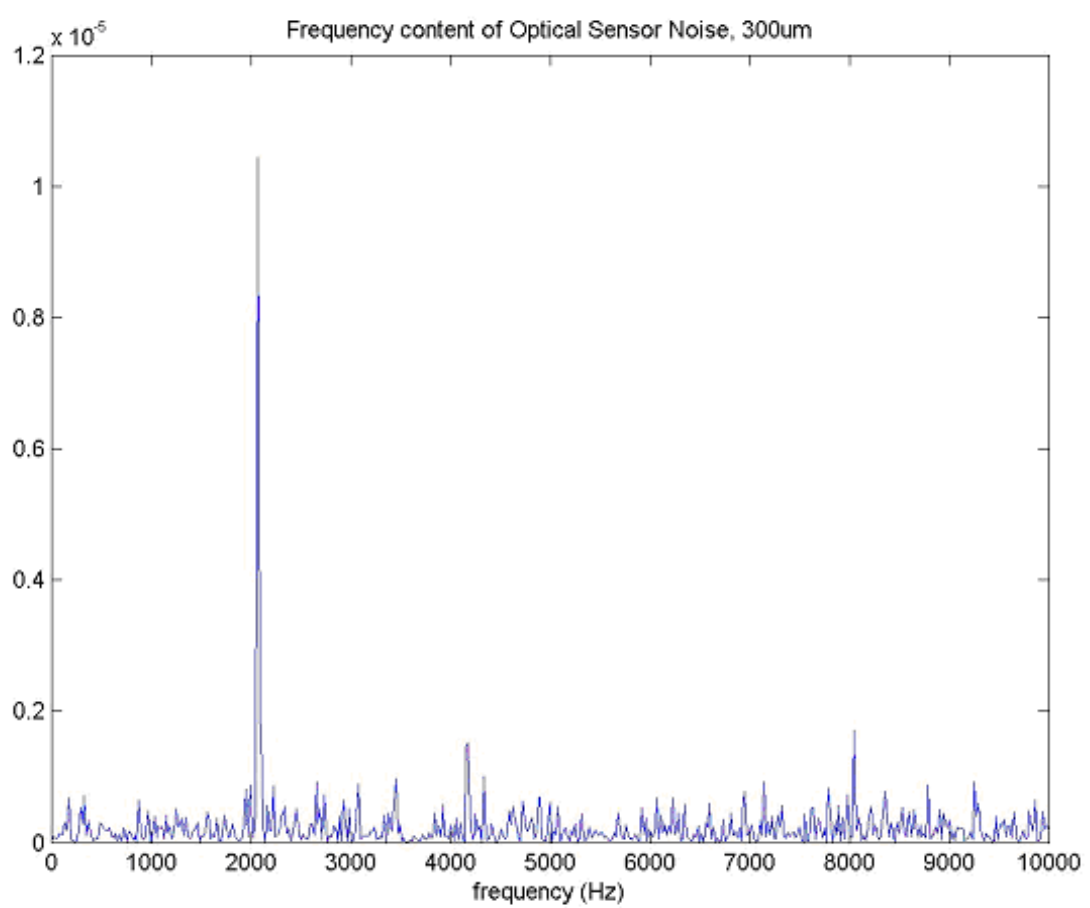

Figure 3.4: Power spectrum of fast Fourier transform of static signal from optical sensor.

\section{Transfer Function Filter; Static Case}

The Transfer function block in MATLAB works very well as a filter with one major drawback; delay. We can "tweak" the coefficients of the powers of "s" in the transfer function block in order to reduce more noise, but we are always adding a delay. A first order low 
pass filter transfer function is of the following form:

$$
\frac{V_{\text {in }}}{V_{\text {out }}}=\frac{1}{\frac{s}{\omega_{0}}+1}
$$

where $\omega_{0}$ is the cut off frequency of the low pass filter. A higher cut off frequency results in less noise filtering, but also less delay, while a lower cut off frequency results in much better filtering, but also an increased delay. For the static case, three different cut off frequencies where explored: $50 \mathrm{~Hz}, 250 \mathrm{~Hz}$, and $400 \mathrm{~Hz}$.

To test this filter, we ran the filter using the three different cut off frequencies against the noisy signal and extrapolating the peak to peak values from the data as well as observing the time it took for the filter to reach its steady state which we record as the delay time. The delay times and filtering ability of the transfer function filter for each selected cut off frequency are shown in Table 3.2 .

Table 3.2: Static noise reduction and delay for a low pass filter using different cut off frequencies.

\begin{tabular}{|r|r|r|}
\hline Cut off Frequency $\mathbf{( H z})$ & Delay(sec) & Noise Peak-to-Peak(V) \\
\hline$\omega_{0}=50 \mathrm{~Hz}$ & .01 & .0003 \\
$\omega_{0}=250 \mathrm{~Hz}$ & .005 & .0008 \\
\hline$\omega_{0}=400 \mathrm{~Hz}$ & .004 & .001 \\
\hline
\end{tabular}

As expected we found that a better filtering, as well as significant delay, occurs at lower values of $\omega_{0}$. The transfer function filter achieves significant noise reduction especially at low cut off frequencies, but the delay will limit the frequency of our experiments. Since the transfer function filter does not have an initial condition parameter, the delay shown is only an initial delay resulting from the instantaneous static input applied to the filter. The dynamic delay throughout the experiment will be dependant on the change in input from one time step to the next. This dynamic delay is found to be much less than the initial delay shown since the time step changes in input value are much less. The investigation of the dynamic behavior of the filter will be explained in more detail later in this section.

\section{Averaging Scheme Filter; Static Case}

The averaging scheme that we developed takes a number of samples specified by the user and averages those samples to produce a value. This limits the operating frequency of 
the system based on the number of samples averaged. Given the sampling rate of the DAQ system, the limiting operating frequency can be calculated: Max_Frequency = $\frac{\text { Computer_Sampling_Rate }}{\# \text { of samples }}$ for example, if we sample at $20 \mathrm{kHz}$, and average over 100 samples we get: $M a x_{-}$Frequency $=\frac{20 \mathrm{kHz}}{100}=200 \mathrm{~Hz}$.

The delay for the averaging scheme is adaptive. The scheme will only average over the current number of available samples if the number of samples chosen by the user is greater than the current number of available samples. For example: If you are averaging over 3 samples, then the first filtered data point produced will be the first data point collected. The second filtered data point produced would be the average of the first and second data point collected. The third averaged point would be the first 3 collected points averaged. The fourth point would be the 2 nd 3 rd and 4th data points averaged together. This adaptive quality produces less delay and should produce fast convergence. Three averaging schemes will be tested which average over different sample ranges: 100 samples, 200 samples, and 375 samples.

To test this filter, we ran the filter using the three different sample numbers against the noisy signal and extrapolating the peak to peak values from the data as well as observing the time it took for each trial to reach its steady state which we record as the delay time. The delay times and filtering ability of the averaging scheme filter for each selected number of samples is shown in Table 3.3.

Table 3.3: Static noise reduction and delay for averaging scheme filter using different sample numbers.

\begin{tabular}{|r|r|r|}
\hline Number of Samples & Delay(sec) & Noise Peak-to-Peak(V) \\
\hline 100 & $<.0025$ & .00034 \\
200 & $<.0025$ & .00018 \\
\hline 375 & $<.0025$ & .00016 \\
\hline
\end{tabular}

\section{Static Results}

Overall the averaging scheme produces less delay, as well as better filtering for the static case than the transfer function filter for the three different trials tested. Using the sensitivity data found from calibration $(.0173 \mathrm{~V} / \mathrm{um})$, the resulting effective resolution for each filter is shown in Table 3.4. The best two averaging scheme filters produce much better resolution 
than the best transfer function filter. The delay from these two averaging schemes are also much less than the transfer function as shown in Table 3.4. Since delay is the main

Table 3.4: Effective resolution and delay for different filters.

\begin{tabular}{|r|r|r|}
\hline Filter Type & Effective Res.(nm) & Impulse Delay (sec) \\
\hline Averaging over 200 samples & $10.3 \mathrm{~nm}$ & $<.0025 \mathrm{sec}$. \\
Averaging over 375 samples & $9.2 \mathrm{~nm}$ & $<.0025 \mathrm{sec}$. \\
\hline Transfer function, with $\omega_{0}=50 \mathrm{~Hz}$ & $16.9 \mathrm{~nm}$ & $.01 \mathrm{sec}$. \\
\hline Transfer function, with $\omega_{0}=250 \mathrm{~Hz}$ & $46.2 \mathrm{~nm}$ & $.005 \mathrm{sec}$. \\
\hline
\end{tabular}

performance measurement, the averaging scheme preforms better for the static case.

\section{Dynamic Filtering}

Testing these filters in a dynamic case was difficult for the averaging scheme. The computation time needed for the averaging scheme increased with the number of samples we chose to average over. The static tests where conducted using a very basic data acquisition system that did not include all the data inputs and did not include any of the outputs since we had no need to drive the system in the static case. When the full system was incorporated, the averaging scheme caused huge lags in the system's computation and forced a reduction in the overall sampling rate for the data. The transfer function filter did not require a huge computation process and would allow the system to run at a much higher sampling rate. The computation time would decrease as we lowered the number of samples we averaged over, but the filtering quality also significantly decreased. Since the averaging schemes computation problem would not allow us to run the experiments at the rate that we needed to run them at, the experiments where all conducted using the transfer function filter.

For the transfer function, the proper cut off frequency needs to be chosen according to the frequency with which we run the experiments. Since we are running experiments over a range of frequencies, we tested different filter parameters against different experimental rates. The experiments presented in this thesis were conducted at linear electrical loading rates of $5 \mathrm{~V} / \mathrm{sec}$ to $500 \mathrm{~V} / \mathrm{sec}$. In order to present the data as systematically as possible, we needed to find the best cut off frequencies that would work throughout this range of loading rates. The behavior of the material and the hysteresis plots produced from the 
data are non-linear and do not change at a predictable frequency. Therefor, the frequency dynamics are unknown and only by visual inspection of filtered vs. unfiltered data could the appropriate filter parameters be found.

To test the effectiveness of the different cut off frequencies of the filter, we looked at a typical strain vs. electric field hysteresis plot and zoomed into an area where the strain value is changing rapidly with the electric field. We focused on this same area of the strain vs electric field hysteresis while increasing the loading rate of the experiment. We then plotted the unfiltered strain signal for each loading rate against filtered data with several different values of the cut off frequency. Figure 3.5 shows the optical sensor signal for the strain measurement plotted against the electric field with a very slow loading rate of $.05 \mathrm{kV} / \mathrm{mm} / \mathrm{sec}$. The unfiltered data along with filtered data using cut off frequencies of $5 \mathrm{~Hz}, 25 \mathrm{~Hz}$, and $100 \mathrm{~Hz}$, are shown. It is clear that for lower values of the cut off frequency $\omega_{0}$ the signal becomes clearer and for this slow experiment none of these filters add any significant delay to the signal.

Figure 3.6 shows the optical sensor signal for the strain measurement plotted against the electric field with a loading rate of $.5 \mathrm{kV} / \mathrm{mm} / \mathrm{sec}$. The unfiltered data along with filtered data using cut off frequencies of $5 \mathrm{~Hz}, 25 \mathrm{~Hz}$, and $100 \mathrm{~Hz}$, are shown similar to Figure 3.5. Just as for the slower experiment in Figure 3.5, the lower values of $\omega_{0}$ produce clearer signals. The $\omega_{0}=5 \mathrm{~Hz}$ filtered signal is smother than the $\omega_{0}=100 \mathrm{~Hz}$ filtered signal. For this faster loading rate, the delays introduced by the $\omega_{0}=5 \mathrm{~Hz}$ filter are clearly observable. The $\omega_{0}=5 \mathrm{~Hz}$ filter will produce undesirable delays for loading rates higher than $.5 \mathrm{kV} / \mathrm{mm} / \mathrm{sec}$ as well. Figure 3.6 shows that the $\omega_{0}=100 \mathrm{~Hz}$ filter does not produce the best noise reduction, but it is the only one of the three that remains centered within the unfiltered signal.

Figure 3.7 shows the optical sensor signal for the strain measurement plotted against the electric field with a loading rate of $5 \mathrm{kV} / \mathrm{mm} / \mathrm{sec}$. The unfiltered data along with filtered data using cut off frequencies of $25 \mathrm{~Hz}, 100 \mathrm{~Hz}$, and $250 \mathrm{~Hz}$. Just as for the slower experiments the lower values of $\omega_{0}$ produce clearer signals as well as introduce more delay. The $\omega_{0}=25 \mathrm{~Hz}$ filtered signal is smother than the $\omega_{0}=250 \mathrm{~Hz}$ filtered signal, but is clearly introducing a large amount of delay. Figure 3.6 shows that the $\omega_{0}=250 \mathrm{~Hz}$ filter does not produce the best noise reduction, but it is the only one of the three that remains fairly 


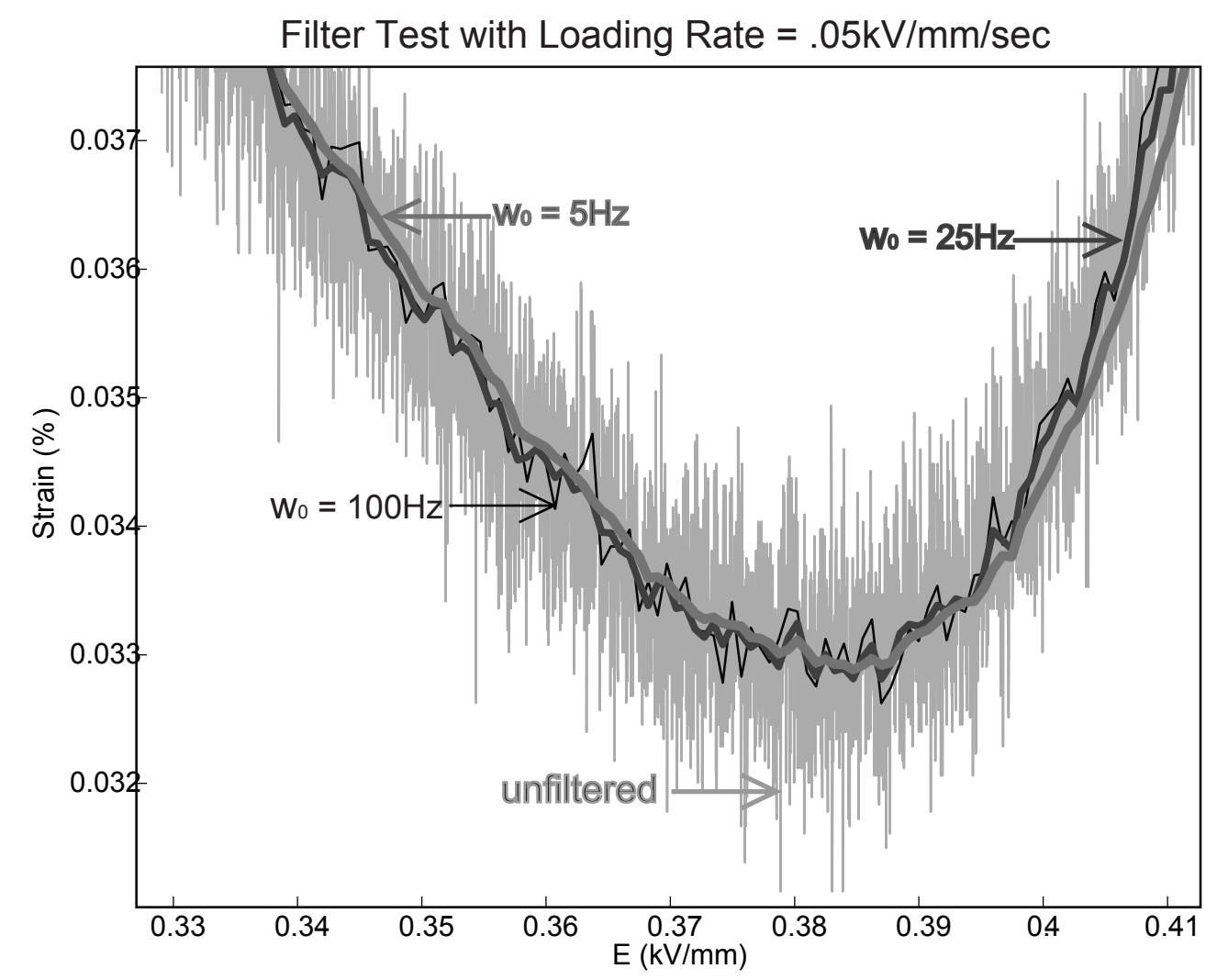

Figure 3.5: Unfiltered vs filtered plots of strain vs electric field for an electric loading rate of $.05 \mathrm{kV} / \mathrm{mm} / \mathrm{sec}$. 


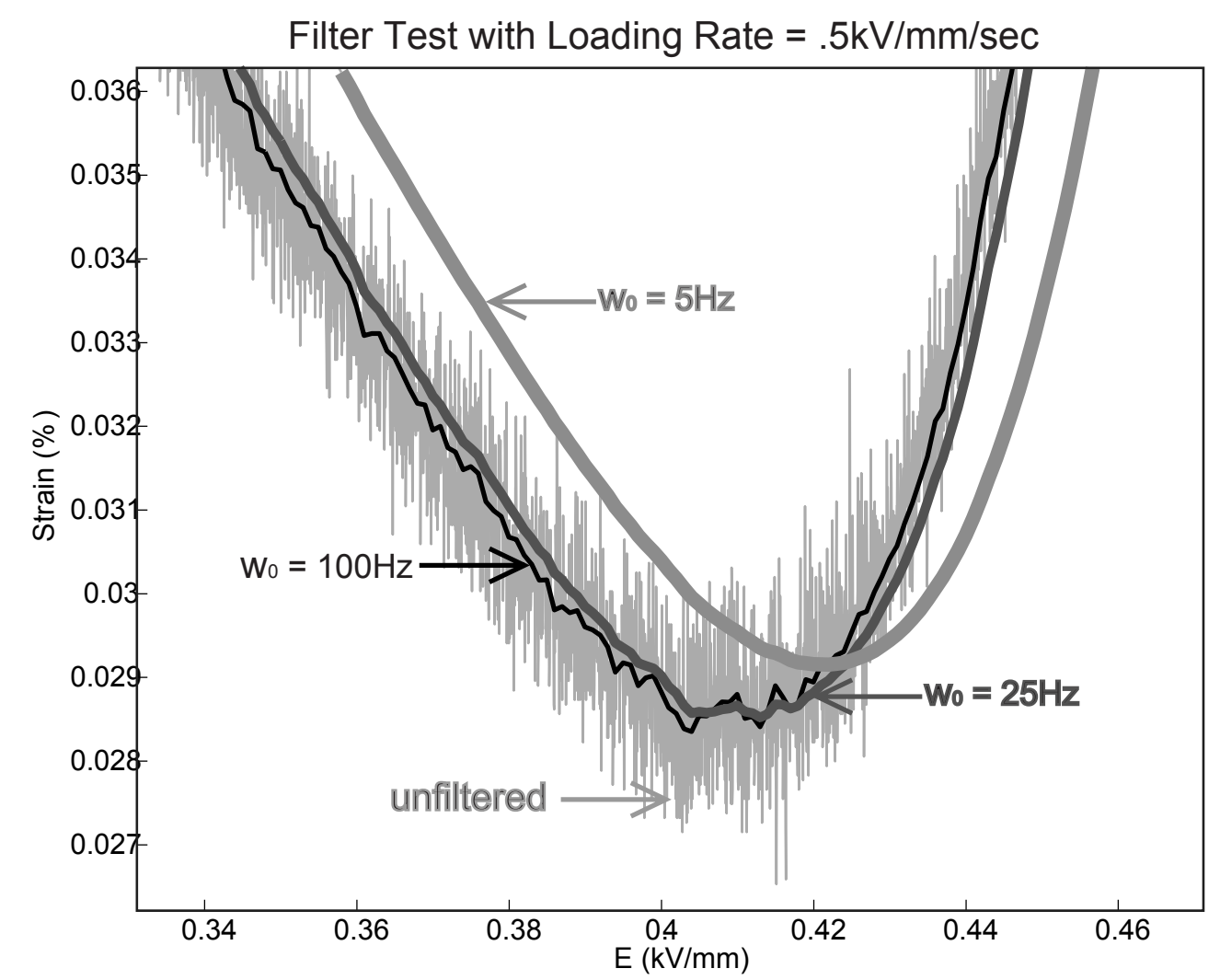

Figure 3.6: Unfiltered vs filtered plots of strain vs. electric field for an electric loading rate of $.5 \mathrm{kV} / \mathrm{mm} / \mathrm{sec}$. 
within the unfiltered signal.

The three loading rates shown in Figures 3.5, 3.6 and 3.7 are the rates for the experiments presented in this thesis. For a particular loading rate, there is a range of cut off frequencies that will allow for significant noise reduction without introducing undesirable delays. Filter tests were also conducted with a wider range of cut off frequencies as well as for higher loading rates for this particular system. Table 3.5 shows the range of cut off frequencies that we found allowable for these experiments as well as the cut off frequencies we choose to use for the $.05 \mathrm{kV} / \mathrm{mm} / \mathrm{sec}, .5 \mathrm{kV} / \mathrm{mm} / \mathrm{sec}$ and $5 \mathrm{kV} / \mathrm{mm} / \mathrm{sec}$ experiments presented in this thesis. At the low end of the frequency range, better noise reduction occurs, and at the high end, less delay will occur. The choice for the proper parameter is determined by the application.

Table 3.5: Range of cut off frequency for filters on different loading rates.

\begin{tabular}{|r|r|r|}
\hline Loading rate $(\mathbf{k V} / \mathbf{m m} / \mathbf{s e c})$ & Cut-off freq. range $(\mathbf{H z})$ & Freq. used in exp. \\
\hline $.05 \mathrm{kV} / \mathrm{mm} / \mathrm{sec}$ & $5 \mathrm{~Hz} \leq \omega_{0} \leq 50 \mathrm{~Hz}$ & $10 \mathrm{~Hz}$ \\
$.5 \mathrm{kV} / \mathrm{mm} / \mathrm{sec}$ & $25 \mathrm{~Hz} \leq \omega_{0} \leq 100 \mathrm{~Hz}$ & $50 \mathrm{~Hz}$ \\
\hline $1 \mathrm{kV} / \mathrm{mm} / \mathrm{sec}$ & $50 \mathrm{~Hz} \leq \omega_{0} \leq 500 \mathrm{~Hz}$ & \\
\hline $5 \mathrm{kV} / \mathrm{mm} / \mathrm{sec}$ & $250 \mathrm{~Hz} \leq \omega_{0} \leq 750 \mathrm{~Hz}$ & $250 \mathrm{~Hz}$ \\
\hline $10 \mathrm{kV} / \mathrm{mm} / \mathrm{sec}$ & $500 \mathrm{~Hz} \leq \omega_{0} \leq 1000 \mathrm{~Hz}$ & \\
\hline $15 \mathrm{kV} / \mathrm{mm} / \mathrm{sec}$ & $750 \mathrm{~Hz} \leq \omega_{0} \leq 2500 \mathrm{~Hz}$ & \\
\hline $20 \mathrm{kV} / \mathrm{mm} / \mathrm{sec}$ & $1000 \mathrm{~Hz} \leq \omega_{0} \leq 5000 \mathrm{~Hz}$ & \\
\hline $40 \mathrm{kV} / \mathrm{mm} / \mathrm{sec}$ & $2500 \mathrm{~Hz} \leq \omega_{0} \leq 10000 \mathrm{~Hz}$ & \\
\hline
\end{tabular}

\subsubsection{Leakage Current}

The PZT actuator is not a perfect capacitor and will have some leakage of current across the actuator. The leakage current effects of a stack pzt actuator are well documented in [31]. The leakage current will result in some error in the experimentation measurements. Leakage current will produce artificial charge buildup across the surface of the reference capacitor which will directly produce artificial changes in the polarization measurement. The amount of error from the leakage current is based on how long the experiment ran for and the leakage rate. The leakage rate is a function of the voltage applied to the surface of the actuator. We were able to determine this relationship by measuring the leakage current at different levels of a constant applied voltages across the actuator. At any given 


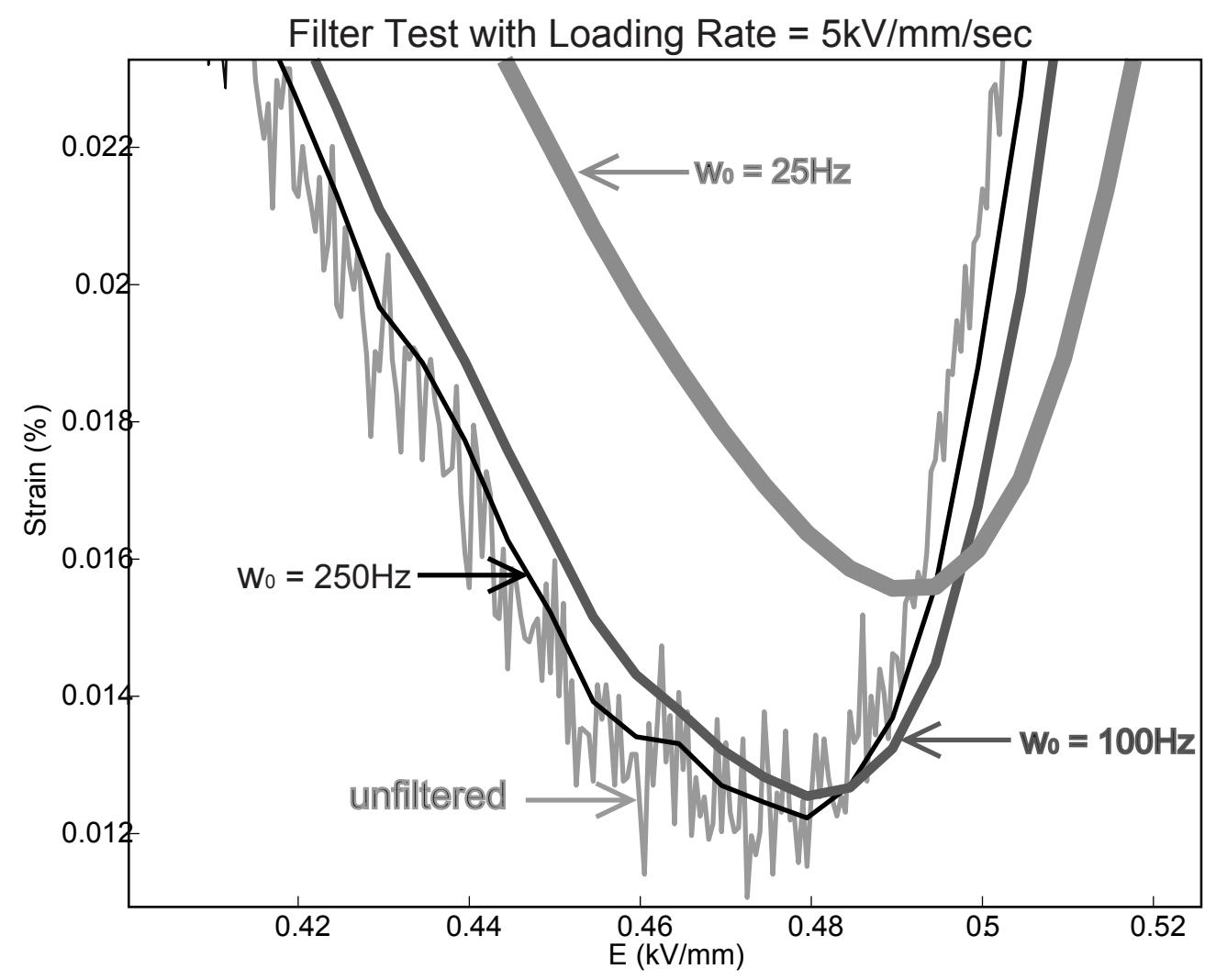

Figure 3.7: Unfiltered vs filtered plots of strain vs. electric field for an electric loading rate of $5 \mathrm{kV} / \mathrm{mm} / \mathrm{sec}$. 
magnitude of the voltage, the material dynamics will need to reach an equilibrium state in order to observe the leakage current only and not any other effects. The material was considered in equilibrium when the strain measurement settled to an equilibrium state and remained constant.

The procedure for this test was to linearly load the voltage, creating an electric field on the actuator, to a constant value. After a period of time, which varied from 3 min. to 20 min. depending on the value of electric field, the strain measurement settled to equilibrium and the polarization was observed to change linearly. From these data sets we fitted several linear curves of leakage, in $\mathrm{C} / \mathrm{mm}$, with respect to time. The slopes of these curves is the leakage rate for that particular voltage. Using those leakage rates from different values of the applied voltage, we produced a curve representing the leakage rate, in $\mathrm{C} / \mathrm{mm} / \mathrm{sec}$, as a function of voltage across the actuator shown in Figure 3.8. Fitting a linear curve to the data points in Figure 3.8 and assuming that the leakage rate is zero in the absence of an applied voltage, the leakage rate can be found as a function of voltage

$$
\text { Leakage_Rate }=3 \times 10^{-7} \times V_{\text {applied }}
$$

Integrating (3.4) will produce the error in the polarization measurement as a function of time. Since the applied voltage is a piecewise linear function, the integration is done in real time in the Matlab/Simulink environment. Since the order of magnitude of the

leakage rate is so small, $10^{-7}$, the leakage current will produce the most error in the longest experiments. An example of the polarization measurement error for the longest experiment presented in this thesis is shown in Figure 3.9. The error has a maximum value of approx. $002 . \mathrm{C} / \mathrm{m}^{2}$, which is two orders of magnitude less than the maximum polarization value. The effect this leakage has on the actual shapes of the hysteresis will be discussed after the experiments have been shown in detail. For our experimental the leakage current is not accounted for.

\subsection{Actuator Behavior under Pure Electrical Loading}

This section summarizes the test procedures of the P802.00 PZT stack actuator under pure electrical loading. Within the capability of the amplifier, both full major hysteresis loops 


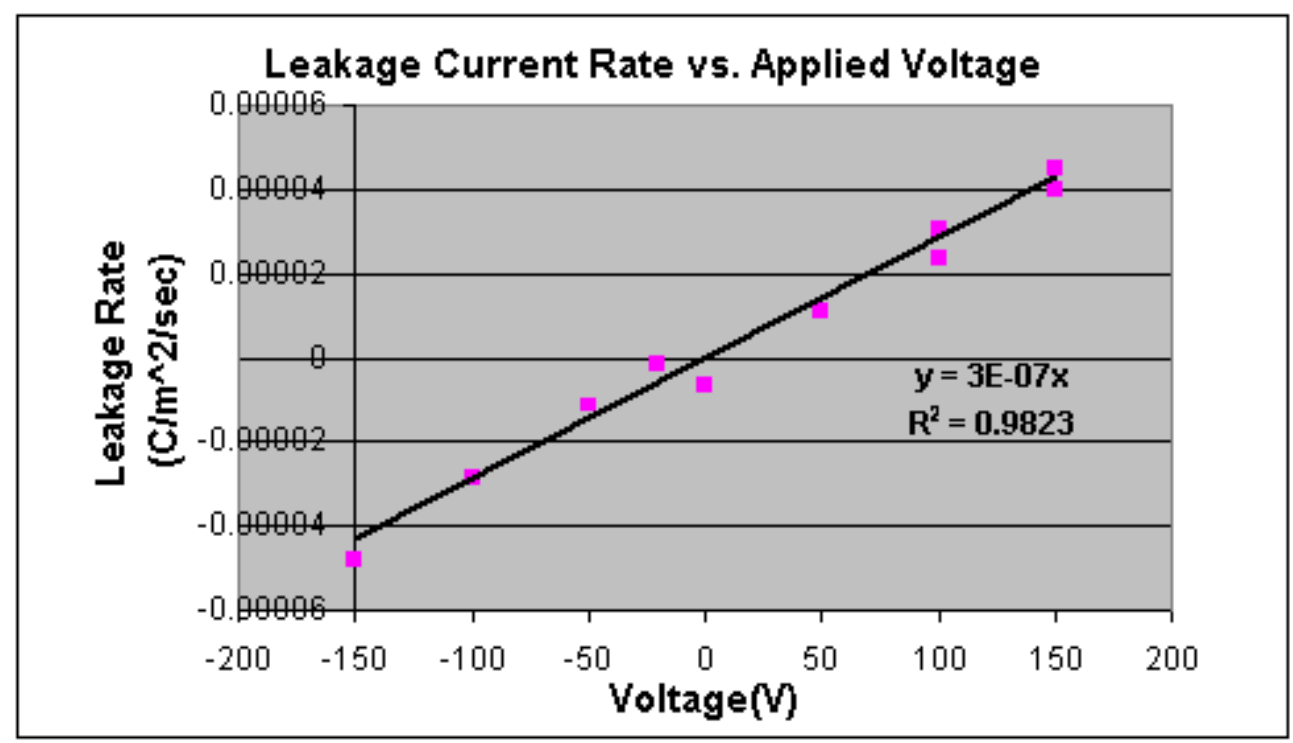

Figure 3.8: Leakage current rate as a function of the applied voltage to the actuator.

and minor-loops are measured with loading rates of $0.05 \mathrm{kV} / \mathrm{mm} / \mathrm{sec}, 0.5 \mathrm{kV} / \mathrm{mm} / \mathrm{sec}$, and $5 \mathrm{kV} / \mathrm{mm} / \mathrm{sec}$. Piecewise linear loads are applied to the actuator to better characterize the polarization vs. field and strain vs. field behavior of the actuator. The domain switching process of the material is investigated through different experimental procedures. Significant rate-dependence of the hysteresis loops are observed from the data. Furthermore, creep-like relaxation behavior under constant electric field is also recorded and analyzed.

\subsubsection{Test Procedures}

The strong hysteretic properties of the material behavior lead to different polarization states at zero electric field depending on the prior history. Ideally, the actuator should start from a state of either full positive or full negative polarization so that the history of the loads can be reliably tracked with respect to the full outer hysteresis loop. In all the experiments, we choose to start from a full positive polarization because the P802 stack actuator has been fully pre-poled to a positive polarization in its manufacturing process. The PIC151 soft PZT can be considered fully poled at an electric field of $2 \mathrm{kV} / \mathrm{mm}$ [3], but as described in 


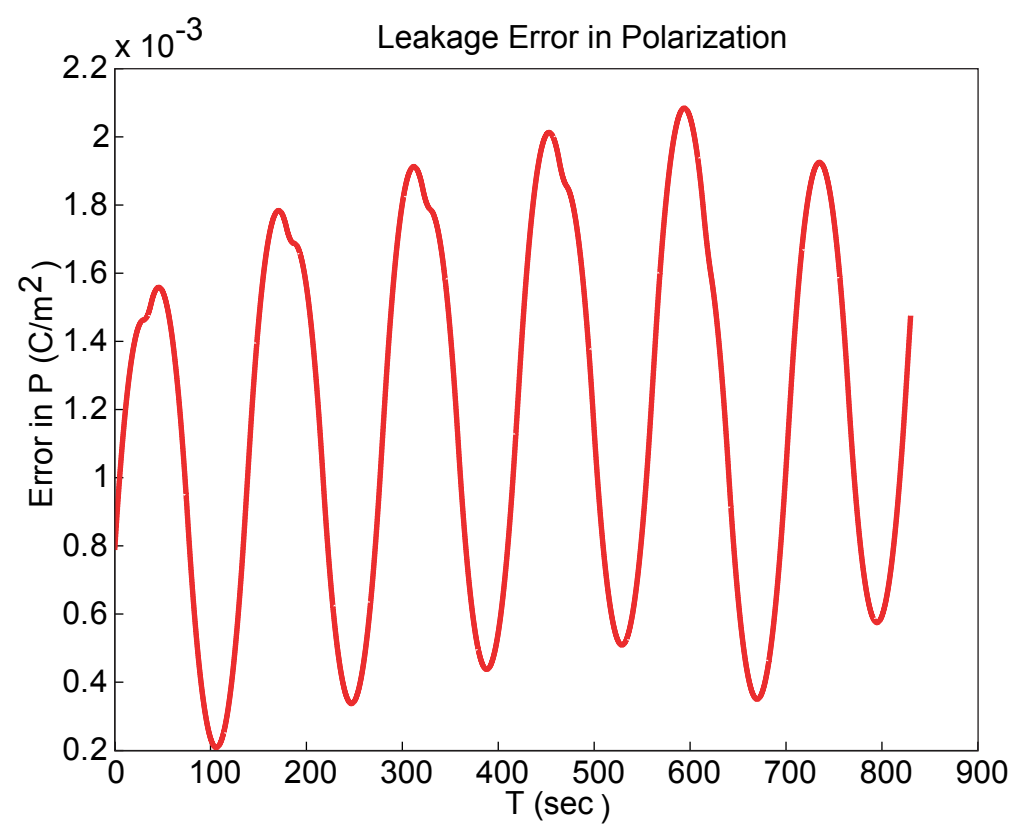

Figure 3.9: Polarization measurement error caused by leakage current for a typical long running experiment. 
section 3.1.1 the actuator has been found to start breaking down after working at this range for an extended period of time. On the other hand, at $1.5 \mathrm{kV} / \mathrm{mm}$, the actuator already begins to show saturation in its phase transformation process, as can be observed in Figure 3.10 , which shows a polarization vs. electric field hysteresis loop between $\pm 1.5 \mathrm{kV} / \mathrm{mm}$.

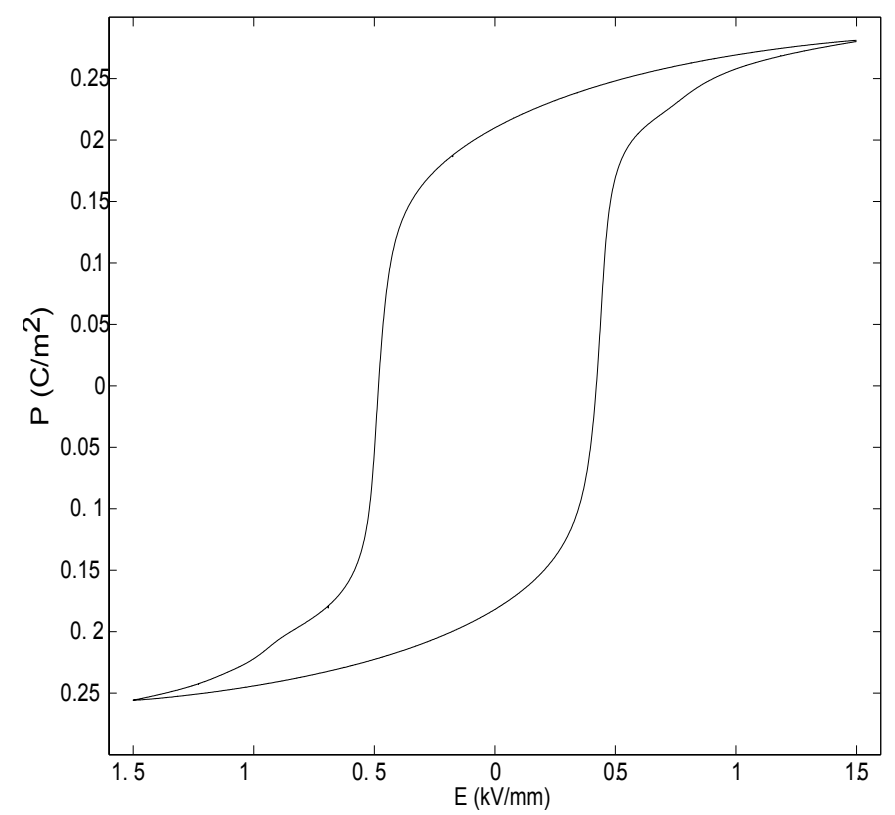

Figure 3.10: Polarization vs. electric field hysteresis loop with cyclic linear electric loading between $\pm 1.5 \mathrm{kV} / \mathrm{mm}$.

Based on these considerations, we always load the actuator to $1.5 \mathrm{kV} / \mathrm{mm}$ and let the actuator saturate at this field before any of the experiments. At the end of the experiments, the electric field is slowly unloaded back to zero. The standard loading procedures for all the experiments are:

1. Discharge the Circuit to remove any charge buildup from previous experiment;

2. Load the actuator from 0 to $1.5 \mathrm{kV} / \mathrm{mm}$ with a slow linear load (5 seconds);

3. Hold the electric field constant at $1.5 \mathrm{kV} / \mathrm{mm}$ for 10 seconds to allow for full polarization; 
4. Slowly load and unload the actuator through a full-loop in order to "warm up" the actuator and remove any memory effects from overnight or long term relaxation;

5. Hold the electric field constant at $1.5 \mathrm{kV} / \mathrm{mm}$ for 5 seconds to again allow for full polarization;

6. Start the experiment from $1.5 \mathrm{kV} / \mathrm{mm}$;

7. Hold the electric field constant at what the experiment ends with for 5 seconds, for relaxation observation;

8. Unload the actuator back to zero field with a slow linear load (5 seconds).

In the above procedures, steps 1 through 5 initialize the PZT to a repeatable maximum positive polarization and step 8 finalizes the experiment. Only the data in steps 6 and 7 are relevant to each experiment and recorded in the post-processing stage on the host computer.

The frequency range of the experiments is mainly limited by the maximum current available from the amplifier. The relationship between maximum amplifier output current, voltage and operating frequency can be approximated as (from PI Website)

$$
i_{\max } \approx f_{\max } \cdot \pi \cdot C_{p z t} \cdot U_{p-p}
$$

where $i_{\max }$ is the peak amplifier current[A], $f_{\max }$ is the maximum sinusoidal operating frequency[Hz], $C_{p z t}$ is the PZT actuator capacitance[F], and $U_{p-p}$ is the peak to peak drive voltage. Therefore the maximum frequency is

$$
f_{\max } \approx \frac{i_{\max }}{\pi \cdot C_{p z t} \cdot U_{p-p}}
$$

The amplifier that we have is limited to a maximum current of $200 \mathrm{~mA}$. In the actuator range from $-40 \mathrm{~V}$ to $150 \mathrm{~V}, U_{p-p}$ is equal to $190 \mathrm{~V}$ and $C_{p z t}$ is approximately $0.8 \mu \mathrm{F}$, so according to Equation $3.6 f \max \approx 0.2 /(\pi \times 0.0000008 \times 190) \approx 418 \mathrm{~Hz}$. For a triangular wave input, $f_{\max }^{\text {lin }} \approx f_{\max } \times \pi / 2 \approx 656 \mathrm{~Hz}$, which converts to a loading rate of $250 \mathrm{kV} / \mathrm{s}$. In the full range from $-150 \mathrm{~V}$ to $150 \mathrm{~V}, f_{\max }=265 \mathrm{~Hz}$ and $f_{\max }^{l i n}=416 \mathrm{~Hz}$, which converts to about $170 \mathrm{kV} / \mathrm{s}$. The maximum loading rate used in this thesis experiments is $500 \mathrm{~V} / \mathrm{s}$, which is well within the systems limits. These are ideal approximations of the maximum rates. In reality, several other conditions limit the maximum operating frequency. 
First, the PZT capacitance can not be considered linear because more current is needed when phase transformation occurs. $0.8 \mu \mathrm{F}$ is the lowest static measurement of $C_{p z t}$. During the experiments, $C_{p z t}$ will be dynamically changing and the maximum frequency will be lower than the theoretical approximation, especially in a full hysteresis loop where a large number of domains are transforming. Second, when the applied voltage switches direction at a reversal point, the current will be changing its sign. The amplifier output will start fluctuating when this sudden change in current combined with the voltage exceeds its power limit. This fluctuation will cause a large error in the output voltage at the reversal points and so should be avoided. Based on these considerations, the maximum reliable rates for the amplifier that we found are shown in Table 3.6.

Table 3.6: Maximum loading rates for different voltage ranges.

\begin{tabular}{|r|r|r|}
\hline & Sinusoidal (Hz) & Linear(V/s) \\
\hline Actuator range (-40V to $150 \mathrm{~V})$ & 100 & 10000 \\
Full range $(-150 \mathrm{~V}$ to $150 \mathrm{~V})$ & 10 & 6000 \\
\hline
\end{tabular}

\subsection{Full-Loop Behavior}

Full Major Hysteresis Loops are the most commonly investigated behavior for piezoelectrical material. The first set of experimental data presented is full-loop behavior under purely linear electrical loading with a loading rate of $0.05 \mathrm{kV} / \mathrm{mm} / \mathrm{sec}$. This results in a fullloop cycling rate of $\frac{0.05 \mathrm{kV} / \mathrm{mm} / \mathrm{sec}}{6 \mathrm{kV} / \mathrm{mm}}=.00833 \mathrm{~Hz}$, which is a relatively slow rate. In these experiments, the strain and polarization are recorder with the results shown In Figure 3.11. Subfigure (a) shows the applied electric field, subfigure (b) and (d) show the Polarization vs. Time and Strain vs. Time respectfully. The Hysteresis loops come from plotting the Polarization vs. Electric Field, shown in subplot (c), and Strain vs. Electric field, shown in subplot (e). The Strain vs. Electric field is typically referred to as the butterfly curve due to its appearance.

Several important characteristics of the material can be seen from the full-loop plots. The obvious hysteretic behavior in subplots (b) and (c) are clear. The asymmetry in these plots stem from the fact that the material was prepoled $+180^{\circ}$ and that we also started at fully poled $+180^{\circ}$. Even though we are loading at a relatively slow rate, not all of 
the crystallites have repoled to $-180^{\circ}$ at $-1.5 \mathrm{kV} / \mathrm{mm}$ and therefor the unloading path is not symmetric to the loading path. This is also why the strain value for the $+1.5 \mathrm{kV} / \mathrm{mm}$ differs from the $-1.5 \mathrm{kV} / \mathrm{mm}$ as can be seen in subfigure(e). Since not all of the domains have reached the $-180^{\circ}$ orientation at $-1.5 \mathrm{kV} / \mathrm{mm}$ and the crystallites that are still in the $90^{\circ}$ phase result in less extension, the resulting overall strain is lower than when all crystallites are fully poled in the $\pm 180^{\circ}$.
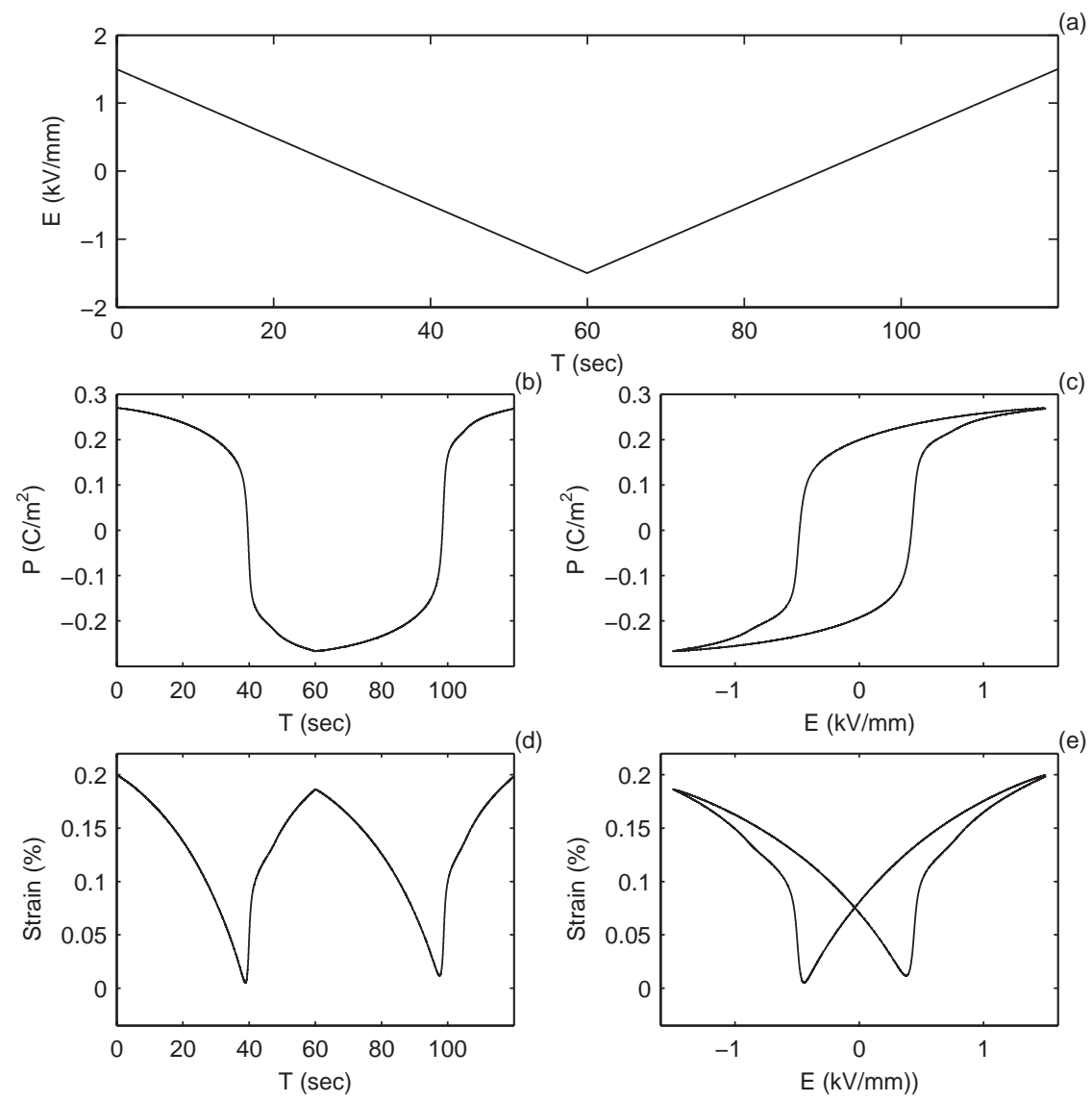

Figure 3.11: Full major loops with a loading rate of $0.05 \mathrm{kV} / \mathrm{mm} / \mathrm{sec}$.

The domain switching process is not very apparent from looking only at the full-loop hysteresis. From the Polarization vs Electric Filed curves in subplot (c) there only appears 
to be two phases, the $+180^{\circ}$ and the $-180^{\circ}$ phase. This is because we are only looking at the charge build up along the $d_{33}$ axes where the $\pm 180^{\circ}$ domains are parallel to. The $90^{\circ}$ domains lie perpendicular to the $d_{33}$ axes. It is not until the Strain vs. Electric Field curve is investigated that the $90^{\circ}$ phase is shown by the strain approaching a minimum during the switching processes. Investigation of the minor-loop behavior of the material will further explore the hysteretic behavior implications of the $90^{\circ}$ phase.

\subsection{Minor-Loop Behavior}

minor-loops are produced by reversing the electric field at different values that lie between $+1.5 \mathrm{kV} / \mathrm{mm}$ and $-1.5 \mathrm{kV} / \mathrm{mm}$. We choose to revers the electric field at points that have different concentrations of $\pm 180^{\circ}$ and $90^{\circ}$ domains within the material occur. The experimental curves will show different behavior of the polarization and strain during for different reversal points.

\subsubsection{First Order Reversals}

First order reversals are produced by starting at the fully $+180^{\circ}$ polarity, at an electric field of $+1.5 \mathrm{kV} / \mathrm{mm}$. The electric field is unloaded at a constant linear rate toward the reversal point. When the reversal point, corresponding to a set electric field value, is reached the electric field is reversed (loading) with the same constant linear rate. When $+1.5 \mathrm{kV} / \mathrm{mm}$ is reached, the electric field is held at $+1.5 \mathrm{kV} / \mathrm{mm}$ for 5 seconds to allow for full $+180^{\circ}$ polarization. From this point another first order reversal curve is produced using the same steps. The constant linear loading rate for this experiment is set at $0.05 \mathrm{kV} / \mathrm{mm} / \mathrm{sec}$ and the reversal points chosen are at $0,-.45,-.50,-.55,-.80$, and $-1.50 \mathrm{kV} / \mathrm{mm}$. The $1.50 \mathrm{kV} / \mathrm{mm}$ reversal corresponds to the full-loop. Figure 3.12 shows the resulting curves from the experimental data. The arrangement for the curves is the same as for the full-loop curves in Figure 3.11. Subfigure (a) is the prescribed electric field, subfigure (b) and (d) show the polarization vs. time and strain vs. time respectfully. The hysteresis curves result from plotting the polarization vs. electric field, shown in subplot (c), and strain vs. electric field, shown in subplot (e).

Referring to subplot (c) in Figure 3.12, the reversal points are choosen at fairly evenly 

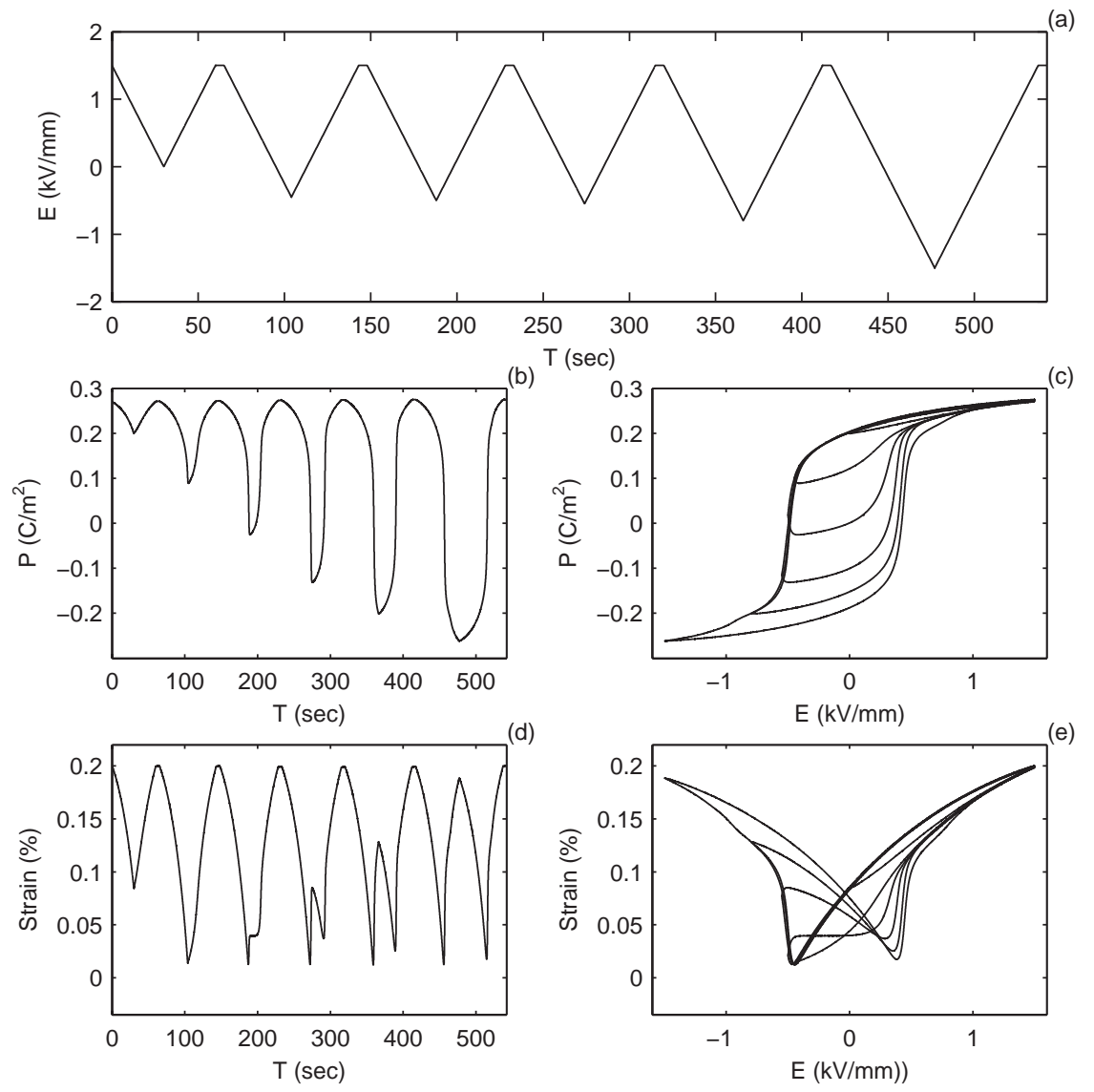

Figure 3.12: First order reversals, with a loading rate of $0.05 \mathrm{kV} / \mathrm{mm} / \mathrm{sec}$. 
spaced values for the polarization. The first reversal point, at $0.0 \mathrm{kV} / \mathrm{mm}$, the majority of the crystals are still in the $+180^{\circ}$ phase. For the second reversal point, at $-.45 \mathrm{kV} / \mathrm{mm}$, some crystals have switched over to the $90^{\circ}$ phase so there is a mixture of $+180^{\circ}$ and $90^{\circ}$ orientations. For the third reversal point, at $-.50 \mathrm{kV} / \mathrm{mm}$, most of the crystals are in the $90^{\circ}$ phase. For the fourth reversal point, at $-.55 \mathrm{kV} / \mathrm{mm}$, some crystals have switched over to the $-180^{\circ}$ phase so there is a mixture of $90^{\circ}$ and $-180^{\circ}$ phase. For the fifth reversal point, at $0.0 \mathrm{kV} / \mathrm{mm}$, the majority of the crystals are in the $-180^{\circ}$ phase. Figure 3.13 , which shows the Polarization vs Electric field and Strain vs. Electric field as well as zooms into the areas of reversal. It can be seen from Figure 3.13 that for both the polarization and strain vs. electric field plots, there is a fairly sharp reversal for the first point at $0.0 \mathrm{kV} / \mathrm{mm}$ where the $180^{\circ}$ phase orientation is preferred. The next reversal, at $-.40 \mathrm{kV} / \mathrm{mm}$, is not as sharp but rather more rounded, noting especially that the polarization continues to fall despite the reversed direction of the electric field. This effect is more clearly seen in the next reversal, at $-.50 \mathrm{kV} / \mathrm{mm}$ where the $90^{\circ}$ phase orientation is preferred. For the $-.55 \mathrm{kV} / \mathrm{mm}$ reversal point the effect is diminished slightly and more resembles the $-.40 \mathrm{kV} / \mathrm{mm}$ reversal and at $-.80 \mathrm{kV} / \mathrm{mm}$ reversal, where the $-180^{\circ}$ phase is dominating, the effect has almost disappeared and the reversal more resembles the $0 \mathrm{kV} / \mathrm{mm}$ reversal.
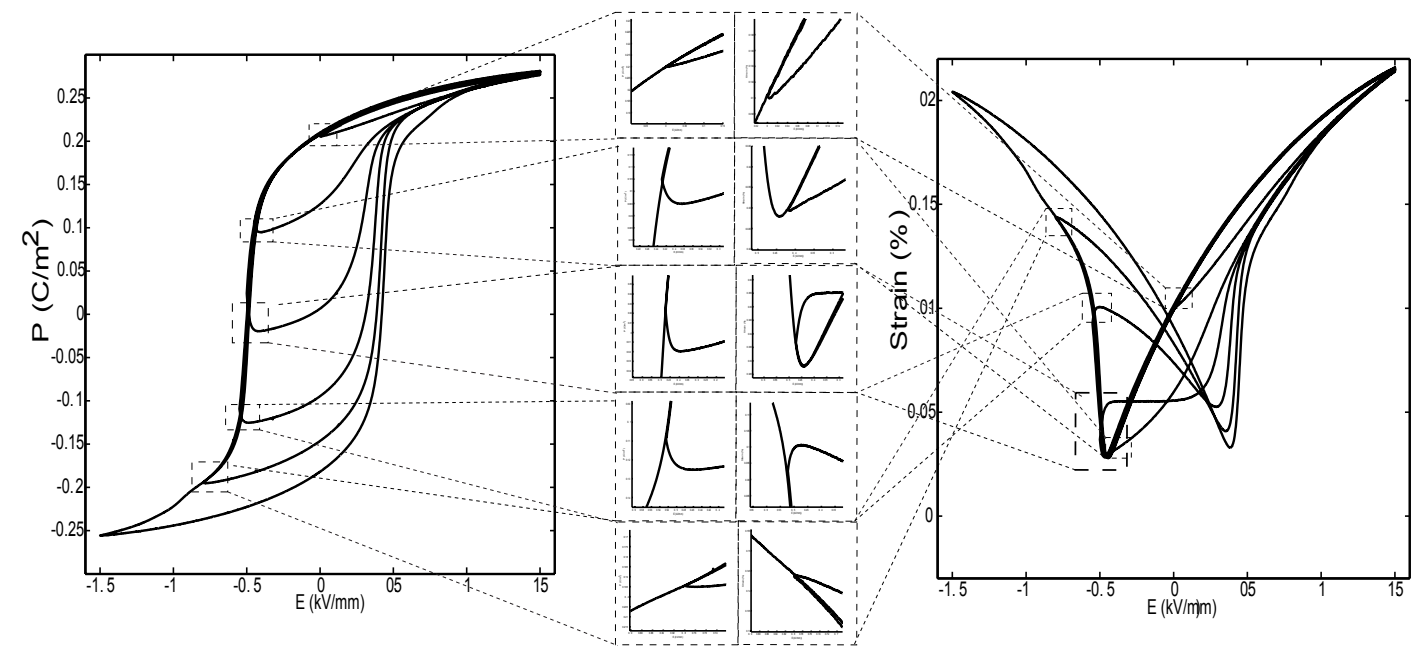

Figure 3.13: First order reversals, polarization vs. electric field and strain vs. electric field with zooms into reversal points. Loading rate of $0.05 \mathrm{kV} / \mathrm{mm} / \mathrm{sec}$. 
The changing reversal behavior as we move through different crystal phases seems to indicate that the different phase orientations have different switching rates. When the crystallites have moved out of the $+180^{\circ}$ phase and into the $90^{\circ}$ phase they continue to move toward the $-180^{\circ}$ phase even though the electric field has been reversed. Moving from the $90^{\circ}$ phase into the $-180^{\circ}$ phase seems to have a slower switching rate than the $+180^{\circ}$ to the $90^{\circ}$ phase that results in a delayed reaction to the reversing electric field.

\subsubsection{Second Order Reversals}

Second order reversals are produced by starting at the fully $+180^{\circ}$ polarity, at an electric field of $+1.5 \mathrm{kV} / \mathrm{mm}$. The electric field is unloaded at a constant linear rate toward $1.5 \mathrm{kV} / \mathrm{mm}$. When $-1.5 \mathrm{kV} / \mathrm{mm}$ is reached the electric field is reversed and reloaded toward the second reversal point. When this second reversal point is reached, the electric field is reversed again toward $-1.5 \mathrm{kV} / \mathrm{mm}$. When $-1.5 \mathrm{kV} / \mathrm{mm}$ is reached the electric field is brought back up to $+1.5 \mathrm{kV} / \mathrm{mm}$ and held there for 5 seconds to allow for full $+180^{\circ}$ polarization. From this repeatable starting point another second order reversal curve is produced using the same steps. The constant linear loading rate for this experiment is set at $0.05 \mathrm{kV} / \mathrm{mm} / \mathrm{sec}$ and the reversal points chosen are at $0,+.35,+.43,+.50,+.80$, and $+1.50 \mathrm{kV} / \mathrm{mm}$. These where chosen to produce fairly evenly spaced reversal curves within the full-loop similar to those seen in the first order reversal plots. Figure 3.14 shows the resulting curves. Subplot (a) is the prescribed electric field, subfigure (b) and (d) show the Polarization vs. Time and Strain vs. Time respectfully. The Hysteresis loops come from plotting the Polarization vs. Electric Field, shown in subplot (c), and Strain vs. Electric field, shown in subplot (e).

Referring to subplot (c) in Figure 3.14, the reversal points occur at fairly evenly spaced values for the polarization, just as for the first order reversals. The first reversal point, at $0.0 \mathrm{kV} / \mathrm{mm}$, the majority of the crystals are still in the $-180^{\circ}$ phase. For the second reversal point, at $+.35 \mathrm{kV} / \mathrm{mm}$, some crystals have switched over to the $90^{\circ}$ phase so there is a mixture of $-180^{\circ}$ and $90 \mathrm{deg}$. For the third reversal point, at $+.43 \mathrm{kV} / \mathrm{mm}$, most of the crystals are in the $90^{\circ}$ phase. For the fourth reversal point, at $-.50 \mathrm{kV} / \mathrm{mm}$, some crystals have switched over to the $+180^{\circ}$ phase so there is a mixture of $90^{\circ}$ and $+180^{\circ}$ phases. For the fifth reversal point, at $0.0 \mathrm{kV} / \mathrm{mm}$, the majority of the crystals are in the $+180^{\circ}$ 

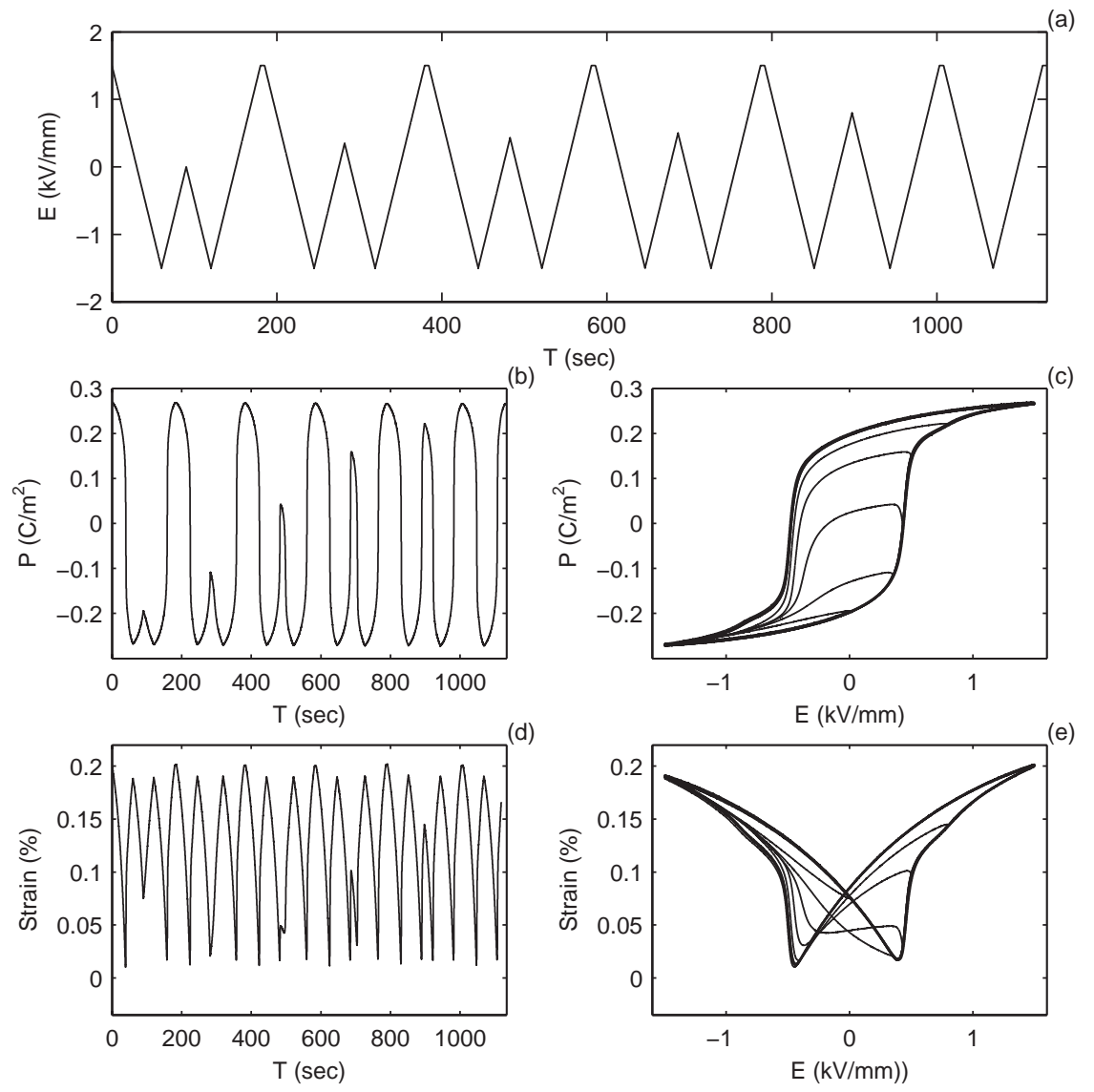

Figure 3.14: Second order reversals, loading rate of $0.05 \mathrm{kV} / \mathrm{mm} / \mathrm{sec}$. 
phase. Figure 3.15, which shows the Polarization vs Electric field and Strain vs. Electric field as well as zooms into the areas of reversal. These results are very similar to those found for the first order reversals in Figure 3.13. It can be seen from Figure 3.15 that for both the polarization and strain vs. electric field plots, there is a fairly sharp reversal for the first point at $0.0 \mathrm{kV} / \mathrm{mm}$ where the $-180^{\circ}$ phase orientation is dominating. The next reversal, at $+.35 \mathrm{kV} / \mathrm{mm}$, is not as sharp but rather more rounded, noting especially that the polarization continues to increase despite the reversed direction of the electric field. This effect is more clearly seen in the next reversal, at $-.43 \mathrm{kV} / \mathrm{mm}$ where the $90^{\circ}$ phase is dominating. For the $+.50 \mathrm{kV} / \mathrm{mm}$ reversal point the effect is diminished slightly and more resembles the $+.35 \mathrm{kV} / \mathrm{mm}$ reversal. At $+.80 \mathrm{kV} / \mathrm{mm}$, where the $+180^{\circ}$ phase is dominating, the effect has almost disappeared and more resembles the $0.0 \mathrm{kV} / \mathrm{mm}$ reversal point.

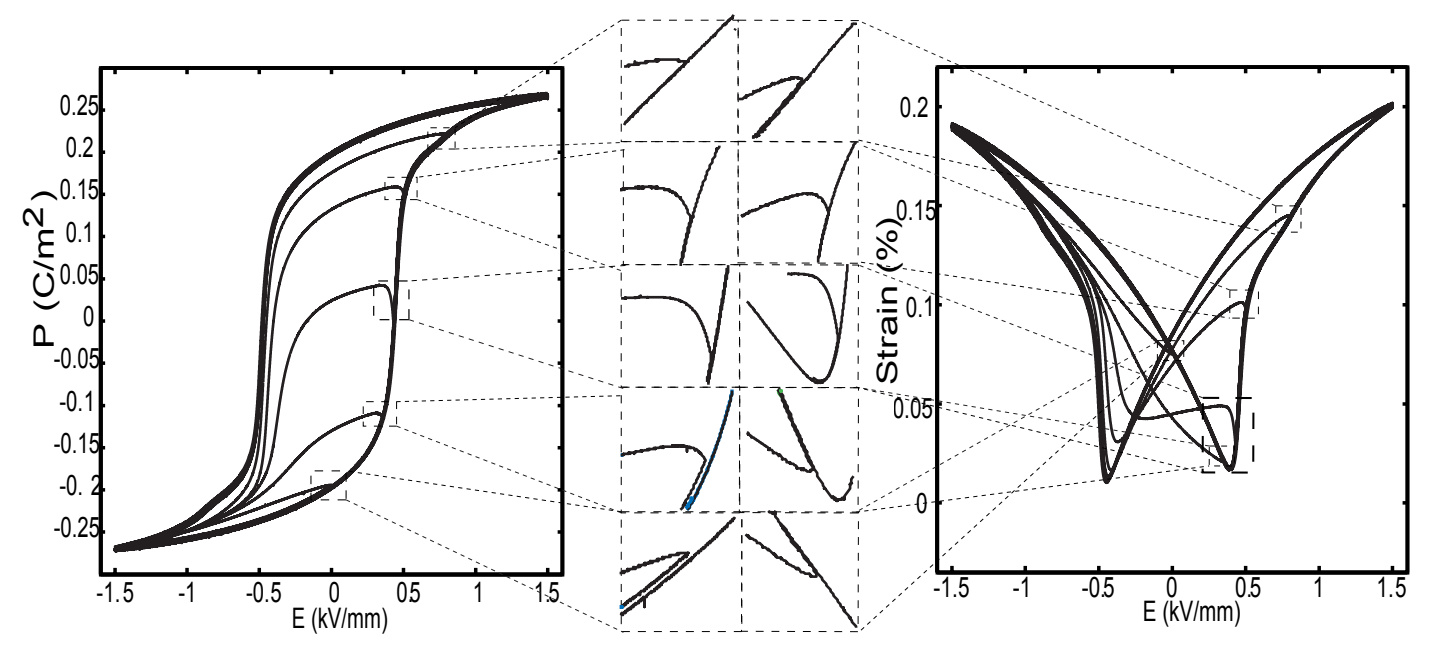

Figure 3.15: Second order reversals, polarization vs. electric field and strain vs. electric field with zooms into reversal points. Loading rate of $0.05 \mathrm{kV} / \mathrm{mm} / \mathrm{sec}$.

The second order reversals show very symmetric behavior to the first order reversals. This shows a symmetry between switching from the $+180^{\circ}$ orientation and switching from the $-180^{\circ}$ crystal orientation. In other words, the crystallites are shown to have symmetric switching behavior for the $\pm 180^{\circ}$ phases. Similarly to that found in the first order reversals, the $90^{\circ}$ switching rate seems to be slower than the $180 \mathrm{deg}$, despite the direction of the 
traveling electric field.

\subsubsection{Third Order Reversals}

Third order reversals are produced by starting at the fully $+180^{\circ}$ polarity, at an electric field of $+1.5 \mathrm{kV} / \mathrm{mm}$. The electric field is unloaded at a constant linear rate toward the reversal point. When the reversal point is reached, the electric field is reversed (loading) with the same constant linear rate and travels $.2 \mathrm{kV} / \mathrm{mm}$ where it is immediately reversed again back toward $-1.5 \mathrm{kV} / \mathrm{mm}$. When $-1.5 \mathrm{kV} / \mathrm{mm}$ is reached the electric field is reversed again back to $+1.5 \mathrm{kV} / \mathrm{mm}$ and held at $+1.5 \mathrm{kV} / \mathrm{mm}$ for 5 seconds to allow for full $+180^{\circ}$ polarization. From this point another third order reversal curve is produced using the same steps. The constant linear loading rate for this experiment is set at $0.05 \mathrm{kV} / \mathrm{mm} / \mathrm{sec}$ and the reversal points chosen are at $0,-.45,-.50,-.55, \&-.80 \mathrm{kV} / \mathrm{mm}$. Figure 3.16 shows the resulting curves. Subplot (a) is the prescribed electric field, subfigure (b) and (d) show the Polarization vs. Time and Strain vs. Time respectfully. The Hysteresis loops come from plotting the Polarization vs. Electric Field, shown in subplot (c), and Strain vs. Electric field, shown in subplot (e).

For third order reversals, particular focus will be on how the small inner loops behave, mainly on if they close and how they deviate from the full-loop. The first reversal point, at $0.0 \mathrm{kV} / \mathrm{mm}$, the majority of the crystals are still in the $+180^{\circ}$ phase. For the second reversal point, at $+.45 \mathrm{kV} / \mathrm{mm}$, some crystals have switched over to the $90^{\circ}$ phase so there is a mixture of $+180^{\circ}$ and $90^{\circ}$ crystal lattice orientations. For the third reversal point, at $+.50 \mathrm{kV} / \mathrm{mm}$, most of the crystals are in the $90^{\circ}$ orientation. For the fourth reversal point, at $+.55 \mathrm{kV} / \mathrm{mm}$, some crystals have switched over to the $-180^{\circ}$ phase so there is a mixture of $90^{\circ}$ and $-180^{\circ}$ crystal orientations. For the fifth reversal point, at $-1.00 \mathrm{kV} / \mathrm{mm}$, the majority of the crystals are in the $-180^{\circ}$ phase. Figure ??, which shows the Polarization vs Electric field and Strain vs. Electric field as well as zooms into the areas of reversal. These results are very similar to those found for the first order reversals in Figure ??. It can be seen from Figure ?? that for both the polarization and strain vs. electric field plots, there is a fairly sharp reversal and the small loop closes for the first point at $0.0 \mathrm{kV} / \mathrm{mm}$ where the $-180^{\circ}$ phase is dominating. The next reversal, at $-.45 \mathrm{kV} / \mathrm{mm}$, is not as sharp but rather more rounded and the small loop no longer closes. This effect is more clearly seen in 

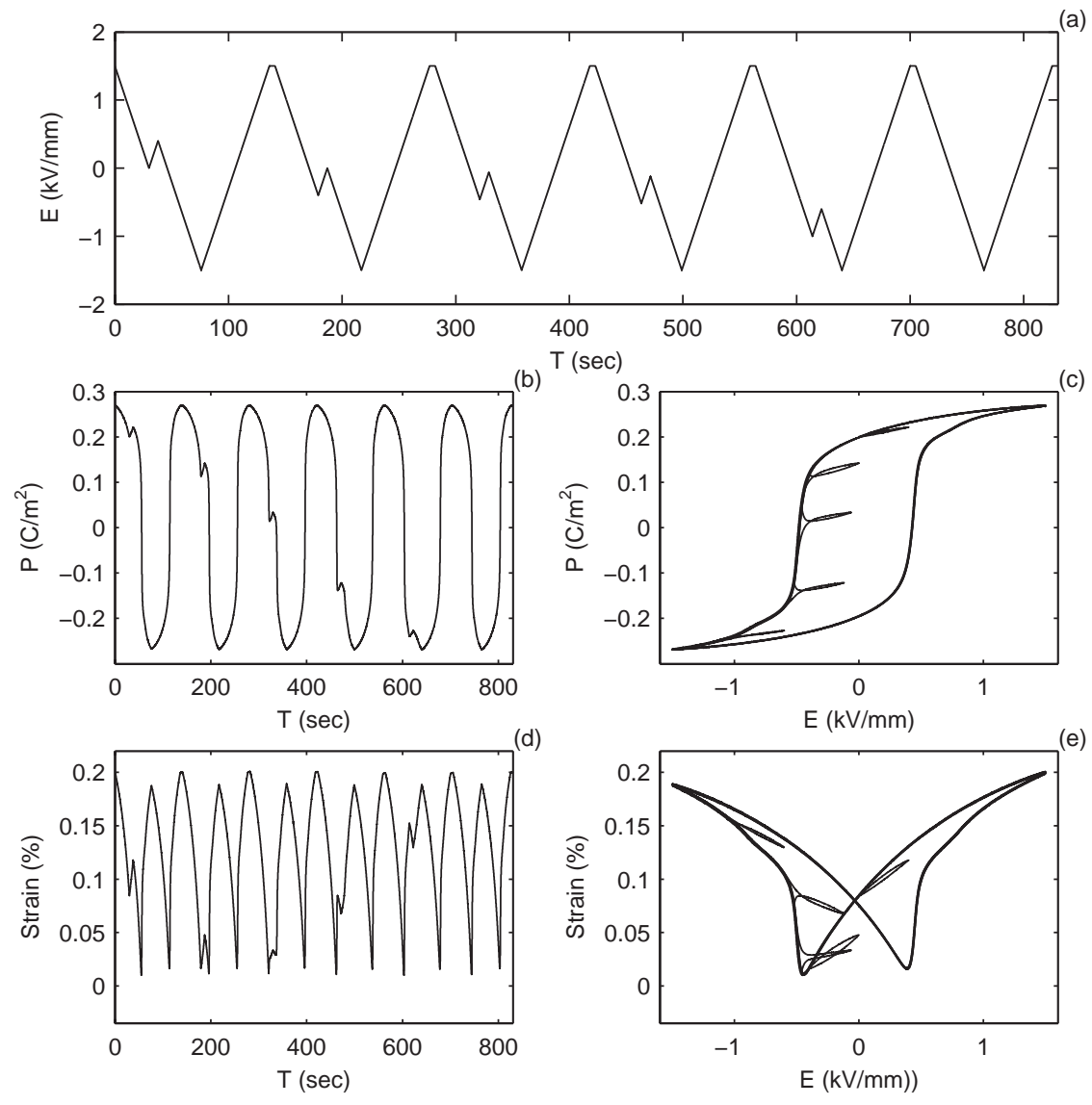

Figure 3.16: Third order reversals, Loading rate of $0.05 \mathrm{kV} / \mathrm{mm} / \mathrm{sec}$ 
the next reversal, at $-.50 \mathrm{kV} / \mathrm{mm}$ where the $90^{\circ}$ phase is dominating, where for the strain, no loop is even formed. For the $-.55 \mathrm{kV} / \mathrm{mm}$ reversal point the effect is diminished slightly and more resembles the $-.45 \mathrm{kV} / \mathrm{mm}$ reversal. At $-1.00 \mathrm{kV} / \mathrm{mm}$, where $+180^{\circ}$ phase is dominating, the effect has almost disappeared and more resembles the $0.0 \mathrm{kV} / \mathrm{mm}$ reversal.
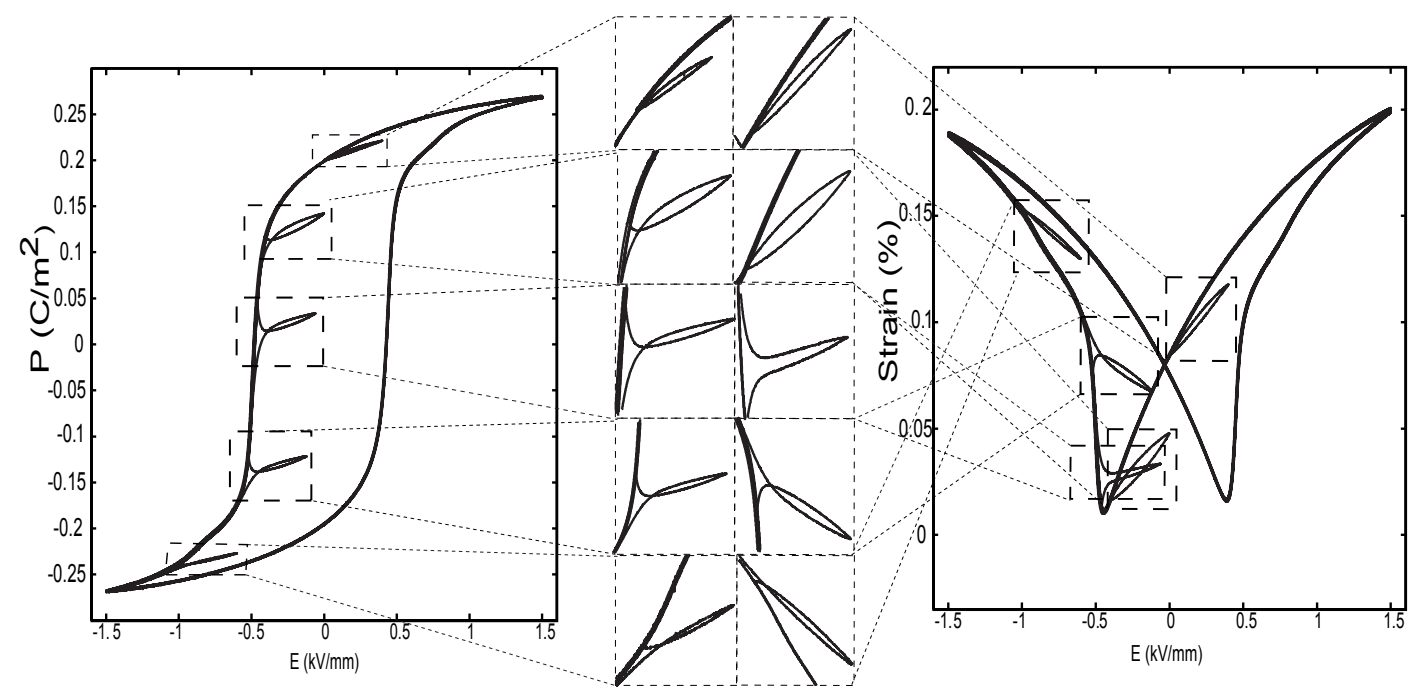

Figure 3.17: Third order reversals, polarization vs. electric field and strain vs. electric field with zooms into reversal points. Loading rate of $0.05 \mathrm{kV} / \mathrm{mm} / \mathrm{sec}$.

From the third order reversals, the effects of reversing the electric field twice during different concentrations of crystallite orientations are shown. The switching process for the $90^{\circ}$ phase is shown to be slower than the $180^{\circ}$ phases. The data shows that crystal domains the $90^{\circ}$ phase have a longer reorientation process which causes a delayed change in strain and polarization values for the changing electric field.

\subsubsection{Fourth Order Reversals}

Fourth order reversals are produced by starting at the fully $+180^{\circ}$ polarity, at an electric field of $+1.5 \mathrm{kV} / \mathrm{mm}$. The electric field is unloaded at a constant linear rate toward $1.5 \mathrm{kV} / \mathrm{mm}$. When $-1.5 \mathrm{kV} / \mathrm{mm}$ is reached the electric field is reversed and reloaded toward the fourth reversal point. When this fourth reversal point is reached, the electric field is reversed again toward $-1.5 \mathrm{kV} / \mathrm{mm}$ and travels $.2 \mathrm{kV} / \mathrm{mm}$ where it is reversed again back 
toward $+1.5 \mathrm{k} / \mathrm{mm}$. When $+1.5 \mathrm{kV} / \mathrm{mm}$ is reached it is held there for 5 seconds to allow for full $+180^{\circ}$ polarization. From this repeatable starting point another fourth order reversal curve is produced using the same steps. The constant linear loading rate for this experiment is set at $0.05 \mathrm{kV} / \mathrm{mm} / \mathrm{sec}$ and the reversal points chosen are at $0,+.35,+.43,+.50,+.80$, and $+1.50 \mathrm{kV} / \mathrm{mm}$. These are the same reversal points as the second order reversals. Figure 3.18 shows the resulting curves. Subplot (a) is the prescribed electric field, subfigure (b) and (d) show the Polarization vs. Time and Strain vs. Time respectfully. The Hysteresis loops come from plotting the Polarization vs. Electric Field, shown in subplot (c), and Strain vs. Electric field, shown in subplot (e).

The fourth order experimental curves show similar results to the third order reversal curves. This reiterates the slower switching rate occurring for the $90^{\circ}$ phase than the $\pm 180^{\circ}$ phases. The symmetry found for the first and second order reversals is also clear for the third and fourth order reversals. This reinforcing the conclusion that switching processes are symmetric for $\pm 180^{\circ}$ phases.

\subsection{Creep Behavior}

When the electric field is held constant, or turned off, the polarization and strain can continue to change. This behavior is referred to as creep or relaxation. Creep behavior results from delayed domain switching. When crystals move out of either $\pm 180^{\circ}$ into the $90 \mathrm{deg}$, they are unable to find equilibrium in the $90^{\circ}$ phase orientation, thus the crystals will continue to reorient themselves even if the electric field is held constant of turned off. Also, the domain switching between the different orientations do not occur at the same rate, and are usually slower than the prescribed electric fields loading rate. Thus, as the electric field loading rate is increased, less of the crystallites have time to switch phase orientations i.e. the crystallites switching processes are unable to keep up with the changing electric potential.

In order to investigate the creep behavior of the material, two sets of experiments are conducted. First, the material is subjected to several different electric field values that are held constant for 15 seconds. The creep behavior at these different electric field potentials is observed. Next, different loading rates are used to reach the constant held electric field 

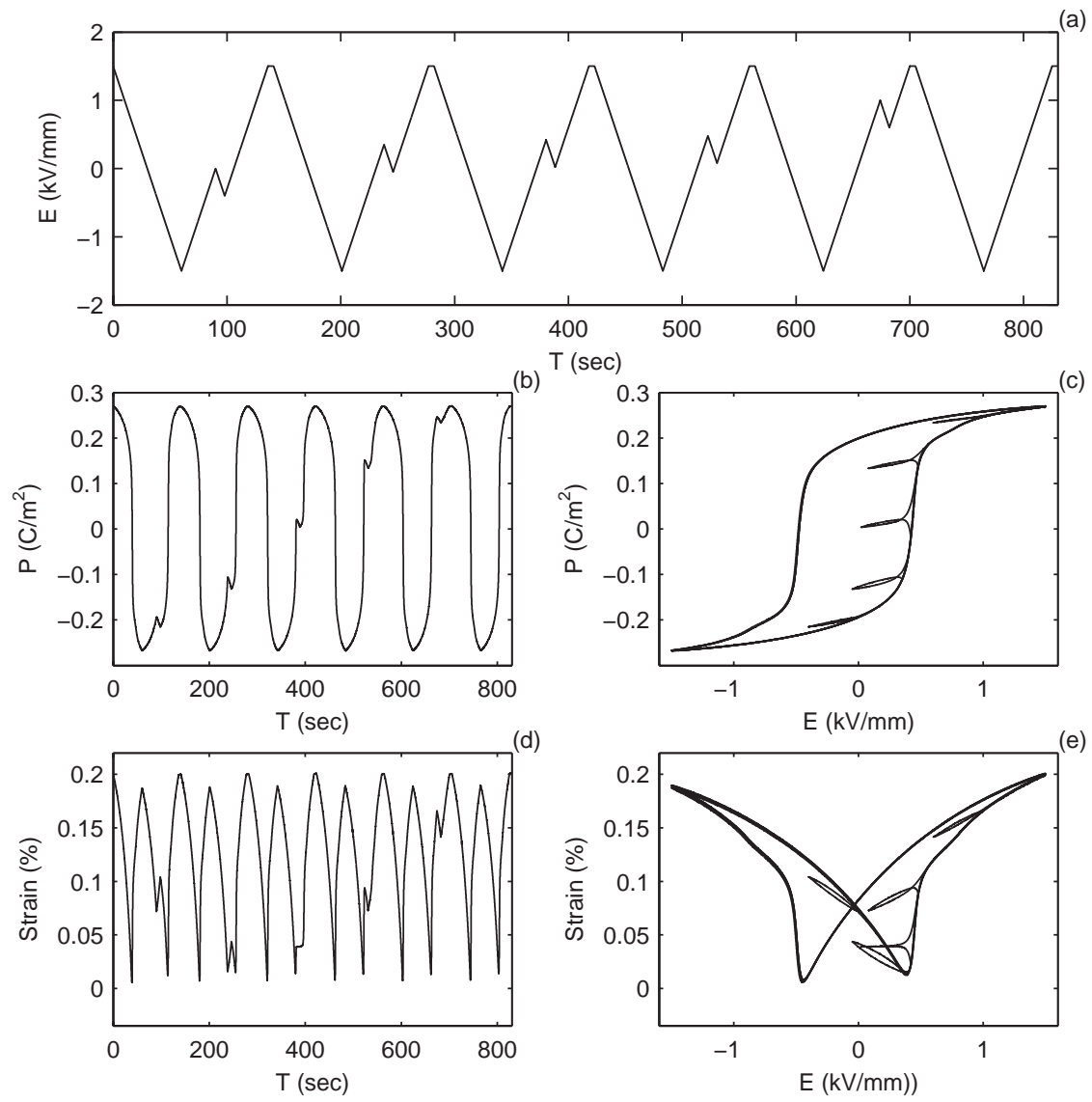

Figure 3.18: Fourth order reversals, loading rate of $0.05 \mathrm{kV} / \mathrm{mm} / \mathrm{sec}$ 

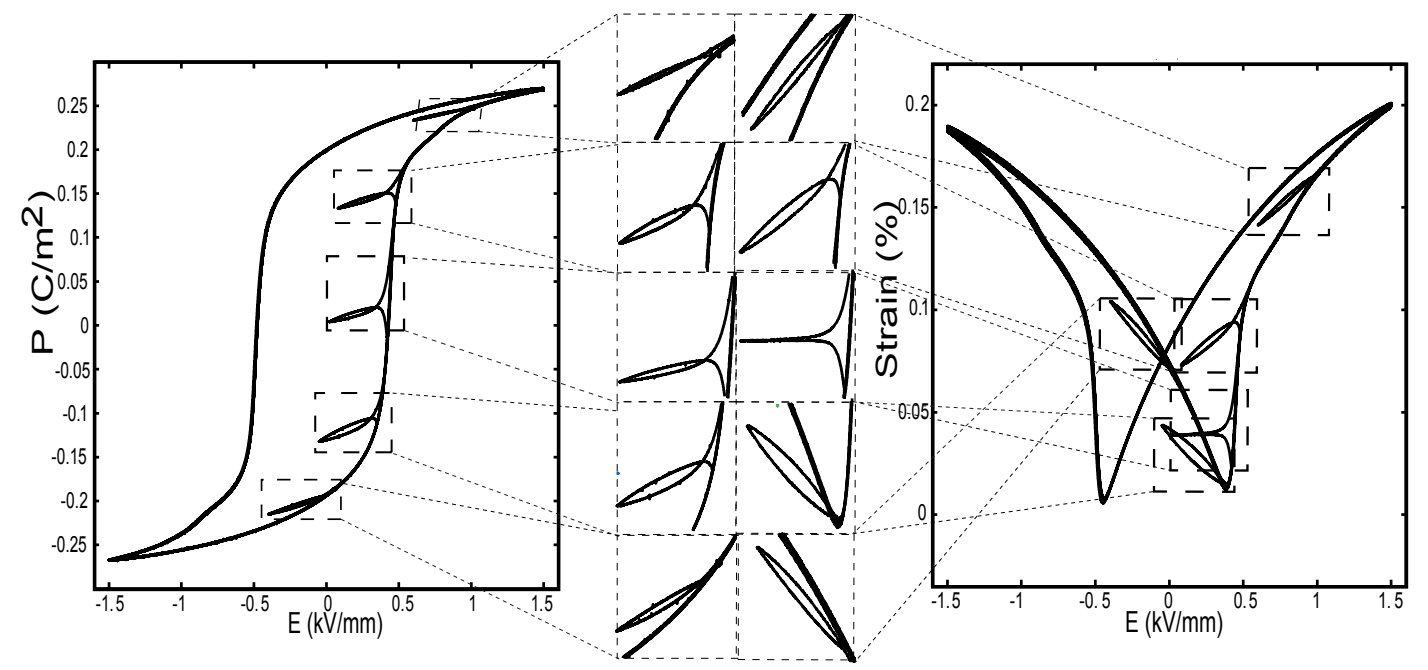

Figure 3.19: Fourth order reversals, polarization vs. electric field and strain vs. electric field with zooms into reversal points. Loading rate of $0.05 \mathrm{kV} / \mathrm{mm} / \mathrm{sec}$.

values and the rate effect of the creep behavior is observed.

Holding the electric field constant at different levels will correspond to different net concentrations of crystallite phase orientations. Studies have suggested that the 90deg. reorientation of domains is expected to be much slower than the $180 \mathrm{deg}$. reorientation of domains [5]. Therefor we would expect that the creep behavior to be greater when more crystallites are in the $90^{\circ}$ phase orientation than the $\pm 180^{\circ}$ orientations. Figure ?? shows Polarization and Strain creep behavior over time for different values of a constantly held electric field.

From Figure ??, the strain and polarization exhibit large creep phenomenon for electric field values that are held constant where the 90deg. phase is dominating. The changes in strain and polarization during the 15 seconds of constant held electric field increase as it moves from the range of preferred $+180 \mathrm{deg}$. orientation and into the range of preferred 90deg. orientation, and then decreases again when it moves from the range of preferred $90^{\circ}$ orientation and into the range of preferred -180 orientation. This clearly suggests that the 90deg. orientation has a slower reorientation process than the $\pm 180^{\circ}$ orientations. This can also be seen in the hysteresis curves shown in Figures 3.21 and 3.22 that are produced from the same creep experimental data 

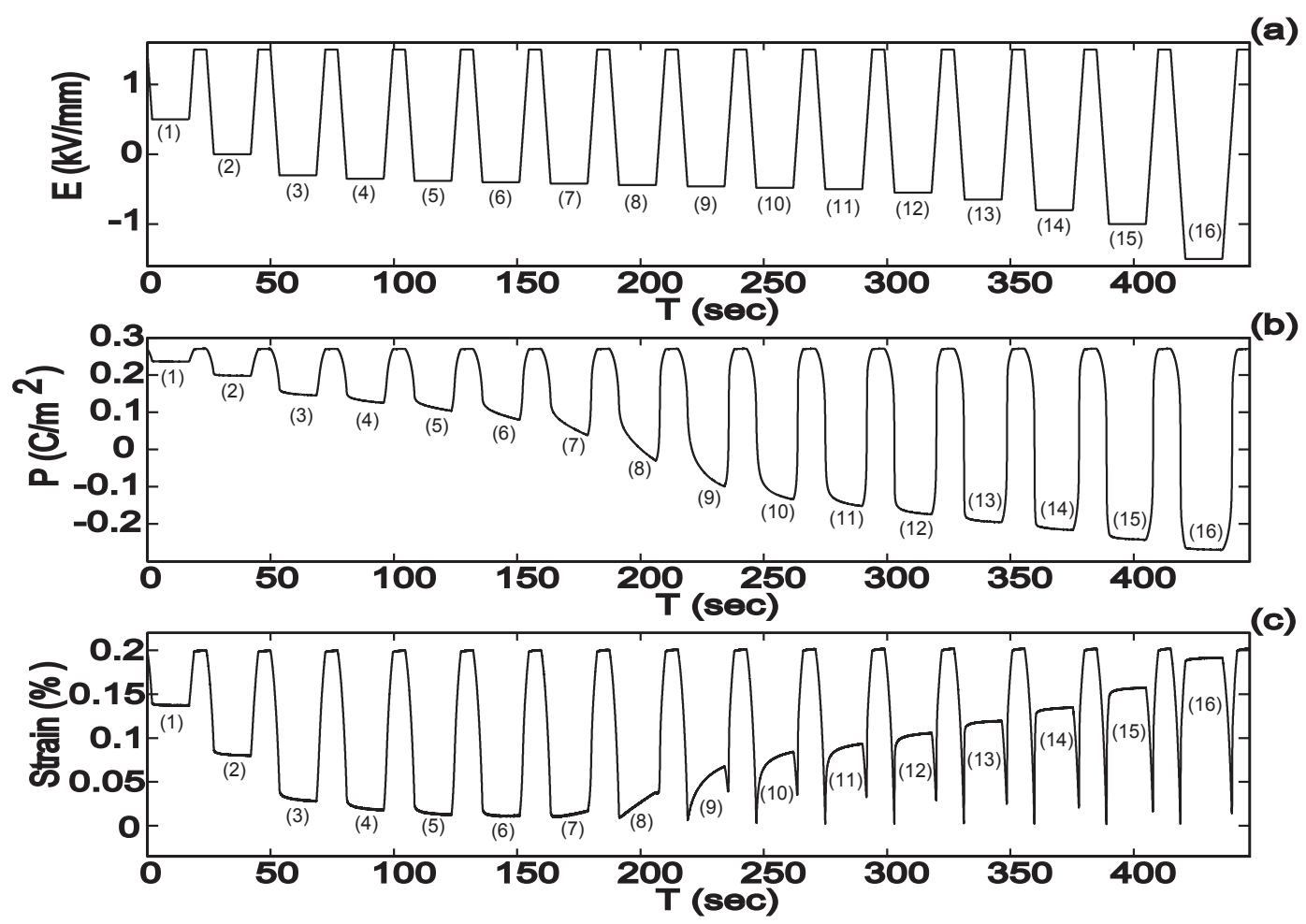

Figure 3.20: Polarization and strain vs time for observation of creep behavior. (a) The applied electric field. (b) Polarization vs. time. (c) Strain vs. time. 


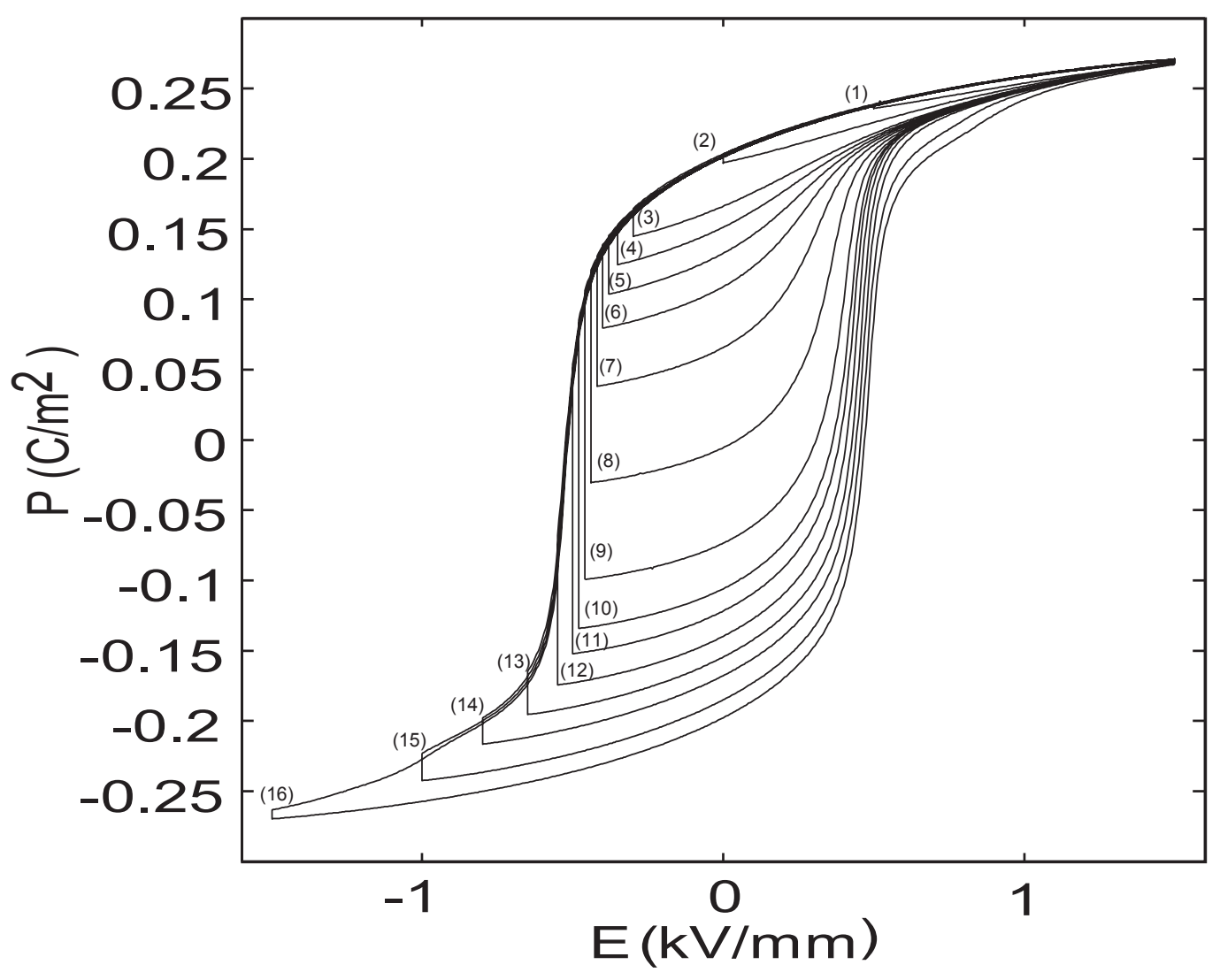

Figure 3.21: Polarization vs. electric field hysteresis for creep experiment. 


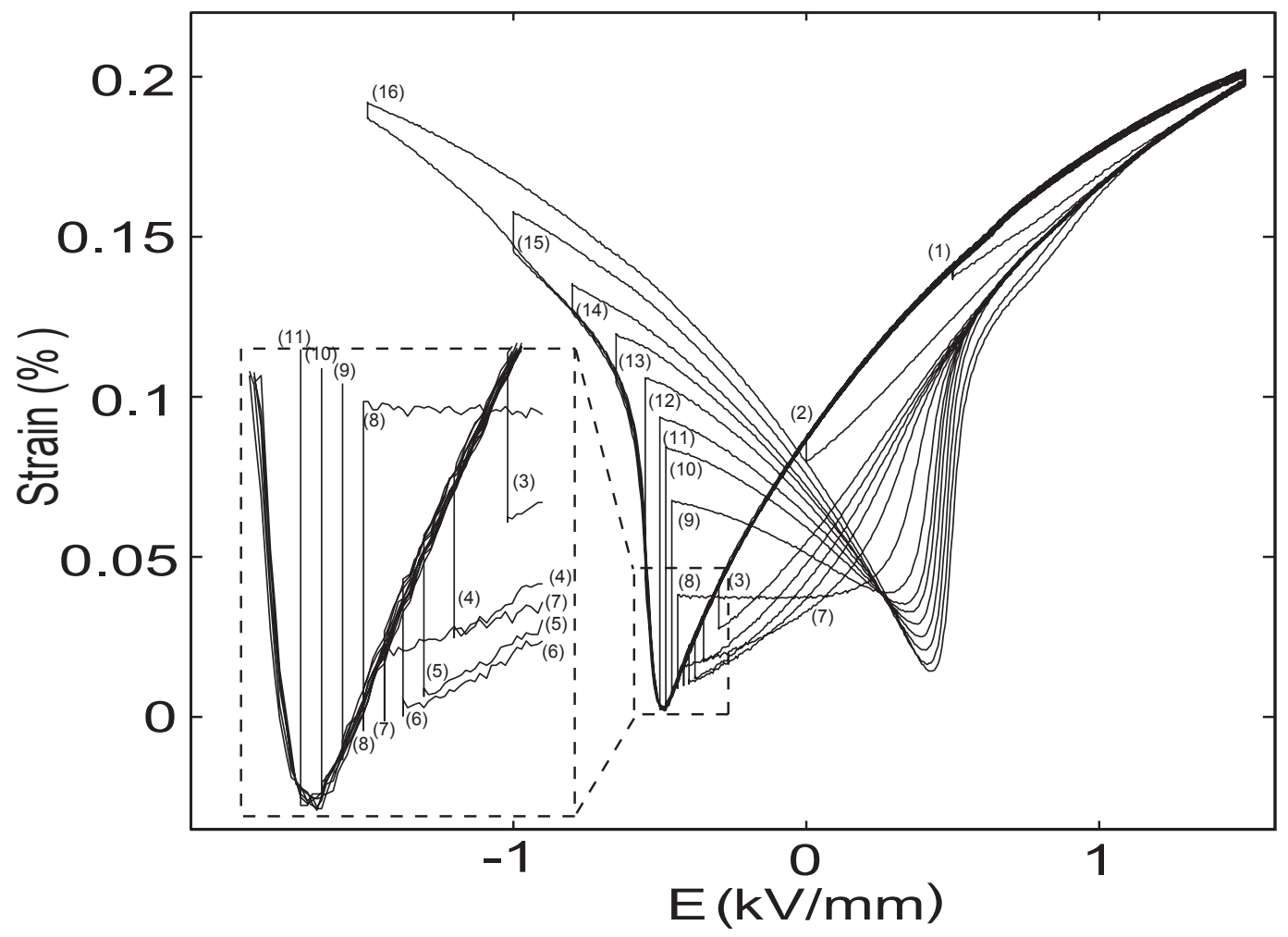

Figure 3.22: Strain vs. electric field hysteresis for creep experiment. 
From Figures 3.21 and 3.22, it is clear that holding electric field at around $-.5 \mathrm{kV} / \mathrm{mm}$ the polarization and strain changes more during the 15 second hold than at values closer to the end points of $\pm 1.5 \mathrm{kV} / \mathrm{mm}$. This behavior agrees with the hysteresis curve behavior for the minor-loop reversal experiments. When the electric field is reversed at a value closer to $-.5 \mathrm{kV} / \mathrm{mm}$, where the majority of the crystallites are in the 90deg. phase orientation, the hysteresis curves show the polarization and strain values continueing to change in the same direction despite the reversed electric field. This suggests that at those points, corresponding to most of the crystals having a $90^{\circ}$ orientation, the material is still heavily reorienting its domains and therefor has a delayed reaction to the electric fields reversal.

\subsection{Rate Dependant behavior}

In this set of experiments, the full-loop and minor-loop experiments conducted in sections 3.3 and 3.4 are repeated at different rates. Loading rates of three orders of magnitude are selected: $0.05 \mathrm{kV} / \mathrm{mm} / \mathrm{sec}, 0.5 \mathrm{kV} / \mathrm{mm} / \mathrm{sec}$, and $5 \mathrm{kV} / \mathrm{mm} / \mathrm{sec}$. These rates correspond to full-loop cycle frequencies of $.00833 \mathrm{~Hz}, .0833 \mathrm{~Hz}$, and $.833 \mathrm{~Hz}$ respectfully. These are all relatively slow rates and fall well within the allowable loading rates for our equipment. The hysteretic behavior for the full-loops and minor-loops are investigated at these different rates.

\subsubsection{Full-Loop}

For the full major loop rate experiments the electric field is loaded and unladed through a full-loop at $0.05 \mathrm{kV} / \mathrm{mm} / \mathrm{sec}, 0.5 \mathrm{kV} / \mathrm{mm} / \mathrm{sec}$ and $5 \mathrm{kV} / \mathrm{mm} / \mathrm{sec}$. Figure 3.23 shows the resulting plots for the full-loop experiment. Subplot (a) is the prescribed electric field, subfigure (b) and (d) show the Polarization vs. Time and Strain vs. Time respectfully. The Hysteresis loops come from plotting the Polarization vs. Electric Field, shown in subplot (c), and Strain vs. Electric field, shown in subplot (e).

From Figure 3.24, we can see that the increasing loading rate results in a widening of the polarization vs electric field hysteresis. This is a result of a delayed switching process of the crystal domains from the faster loading rates. This indicates that the switching process is clearly rate dependant. From Figure 3.25, the strain vs electric field hysteresis at the 

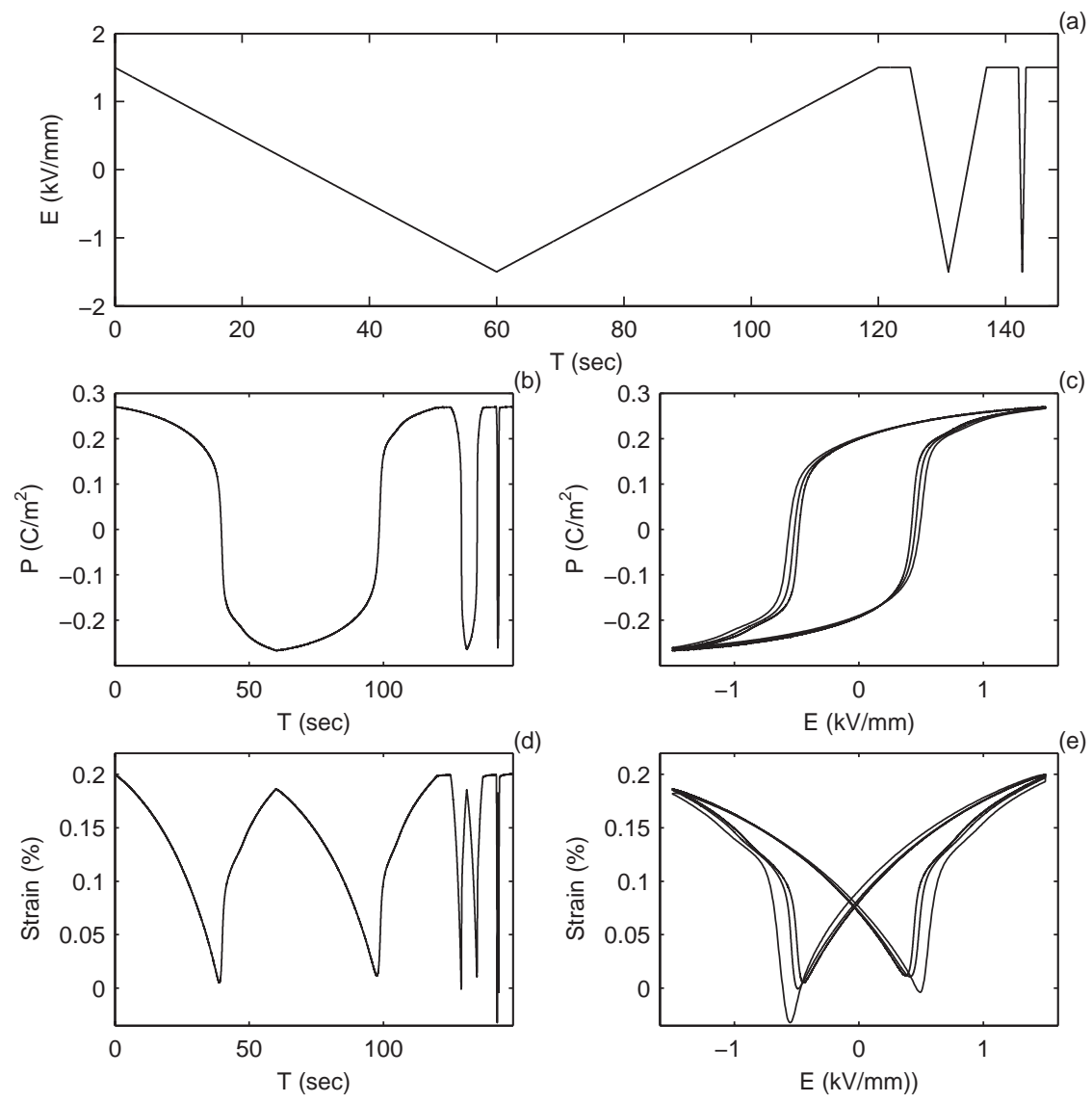

Figure 3.23: Full-loops, loading rates of $0.05 \mathrm{kV} / \mathrm{mm} / \mathrm{sec}, 0.5 \mathrm{kV} / \mathrm{mm} / \mathrm{sec}$, and $5 \mathrm{kV} / \mathrm{mm} / \mathrm{sec}$. 


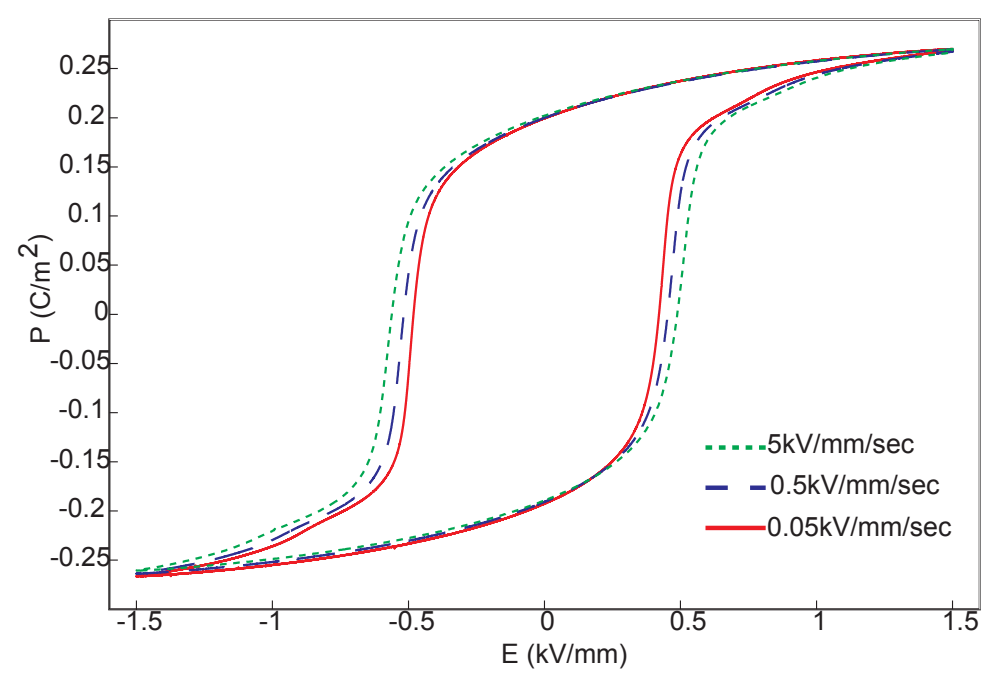

Figure 3.24: Full-loop polarization vs. electric field hysteresis at different loading rates.

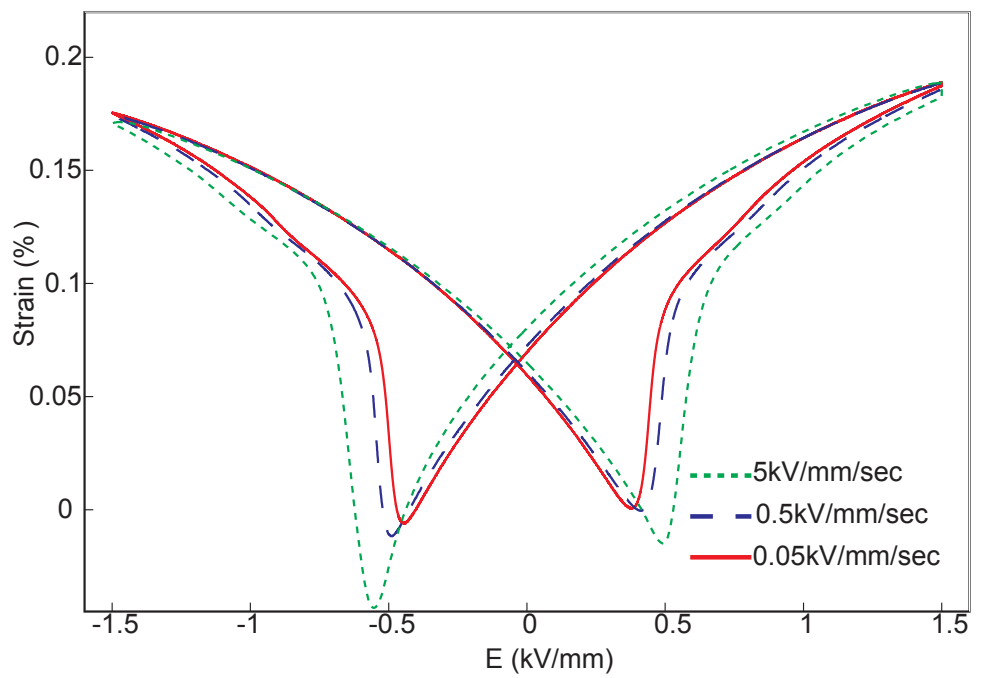

Figure 3.25: Full-loop strain vs. electric field hysteresis at different loading rates. 
different loading rates, a larger extension of the material occurs with higher loading rates - Since $\mathrm{a}+180^{\circ}$ orientation and $\mathrm{a}-180^{\circ}$ orientation would symmetrically have the same strain value, the larger extension seems to be resulting from the $90^{\circ}$ orientation. This is an indication of a larger percentage of crystals in the $90^{\circ}$ phase at any particular time. This would fit a slower reorientation rate for the $90^{\circ}$ phase. When the loading rate is fast, all of the crystals are able to move out of the $\pm 180^{\circ}$ phase (depending on the direction of the electric field) quickly, which results in almost all of the crystals to move into the $90^{\circ}$ phase at once. Since the reorientation for $90^{\circ}$ phase is slower than for the $\pm 180^{\circ}$ phase, the crystals take more time to move into the $\pm 180^{\circ}$ orientations. Thus, at faster loading rates, a larger percentage of crystals are in the $90^{\circ}$ orientation at one particular time. The $90^{\circ}$ orientation result in a shorter strain because the center ion is no longer aligned in the $\mathrm{d}_{33}$ plain as shown in chapter 1 . On the other hand, when the loading rates are slow, the time between individual crystals switching out of the $\pm 180^{\circ}$ phase (depending on the direction of the electric field) is slow, and less of the crystals are able to enter the $90^{\circ}$ phase in a given time. Therefore, by the time it takes all of the crystals to leave the $+180^{\circ}$ phase, many of them have also already left the $90^{\circ}$ phase and are in the $-180^{\circ}$ phase. Thus, there is less of a percentage of crystals in the $90^{\circ}$ phase at any one time which does not allow for the shortest actuator extension.

\subsubsection{First Order Reversals}

For the first order reversals in Figure 3.26, the polarization vs electric field hysteresis curves are shown for different loading rates in subplot (a) (b) and (c) which have electric field loading rates of $.0 .05 \mathrm{kV} / \mathrm{mm} / \mathrm{sec}, 0.5 \mathrm{kV} / \mathrm{mm} / \mathrm{sec}$ and $5 \mathrm{kV} / \mathrm{mm} / \mathrm{sec}$ respectfully. Likewise, subplots (d), (e), and (f) show the strain vs electric field hysteresis curves at the same rates, respectfully. Figure 3.26 shows that as the loading rate is increased, the polarization and strain minor-loops have shorter strokes. This is a result of the delayed reaction to increasingly faster loading rates similarly found for the full-loop hysteresis curves. Essentially, the reorientation process of the crystalline domains is unable to keep up with the increased loading rates resulting in a shorter stroke for the strain and likewise a smaller change in polarization. This delayed reaction does not have as much effect on reversals that occur

where the majority of the materials crystalline are in either $\pm 180^{\circ}$ orientations, as can be 
seen from the first and fifth minor-loops, whose positions are relatively unaffected by the increasing loading rate. This suggests that the $90^{\circ}$ phase is more effected by different loading rates.
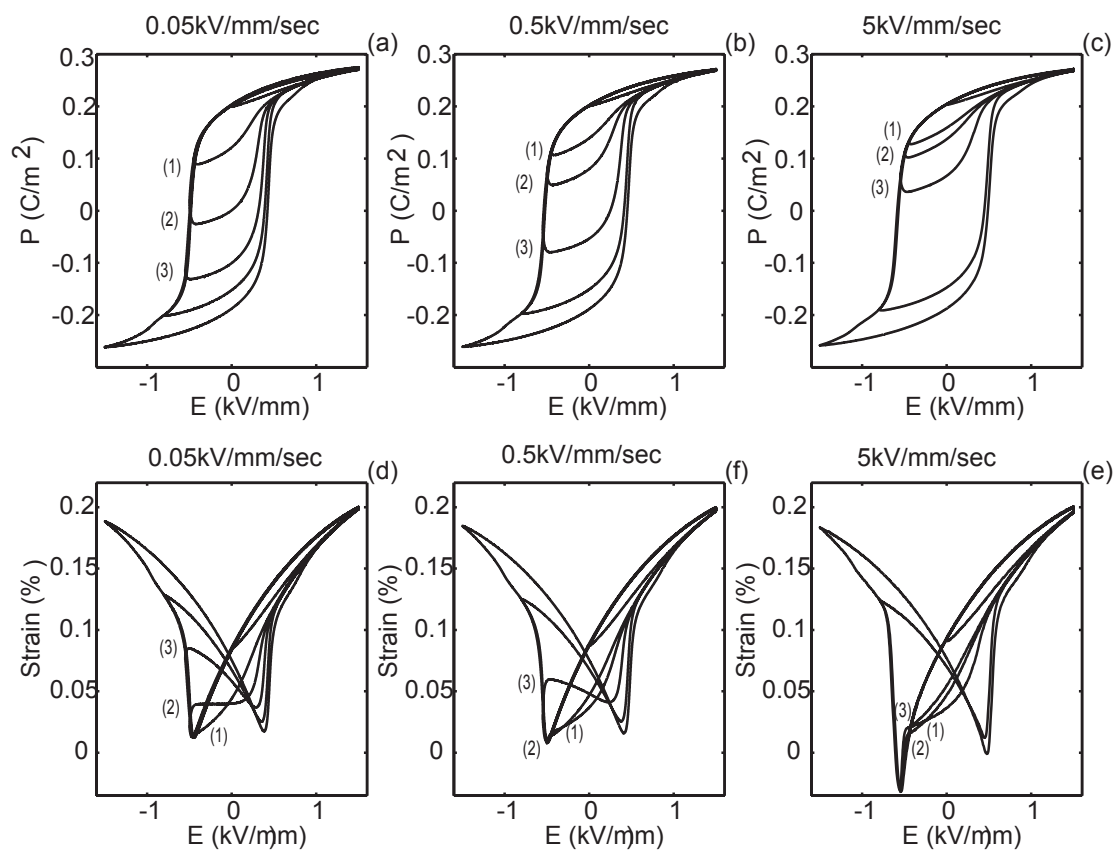

Figure 3.26: First-order inner-loop hysteresis for different loading rates. (a-c) Polarizatin vs electric field. (d-f) Strain vs electric field.

If we look at the creep behavior for the different rates when we hold the electric field constant at the same values used for the first order reversal points shown in Figure 3.27, the strain and polarization appear to achieve similar values after the 15 second hold regardless of loading rate. This suggests that for a particular value of the electric field, the strain and polarization will creep toward a particular equilibrium given enough time. This also reiterates the dependence of loading rate on the materials hysteretic behavior and domain switching process.

Comparing the curves in Figure 3.26 and in Figure 3.27, the strain values show a consistant motion toward the same equilibriums for the same values of constatnly held electric 

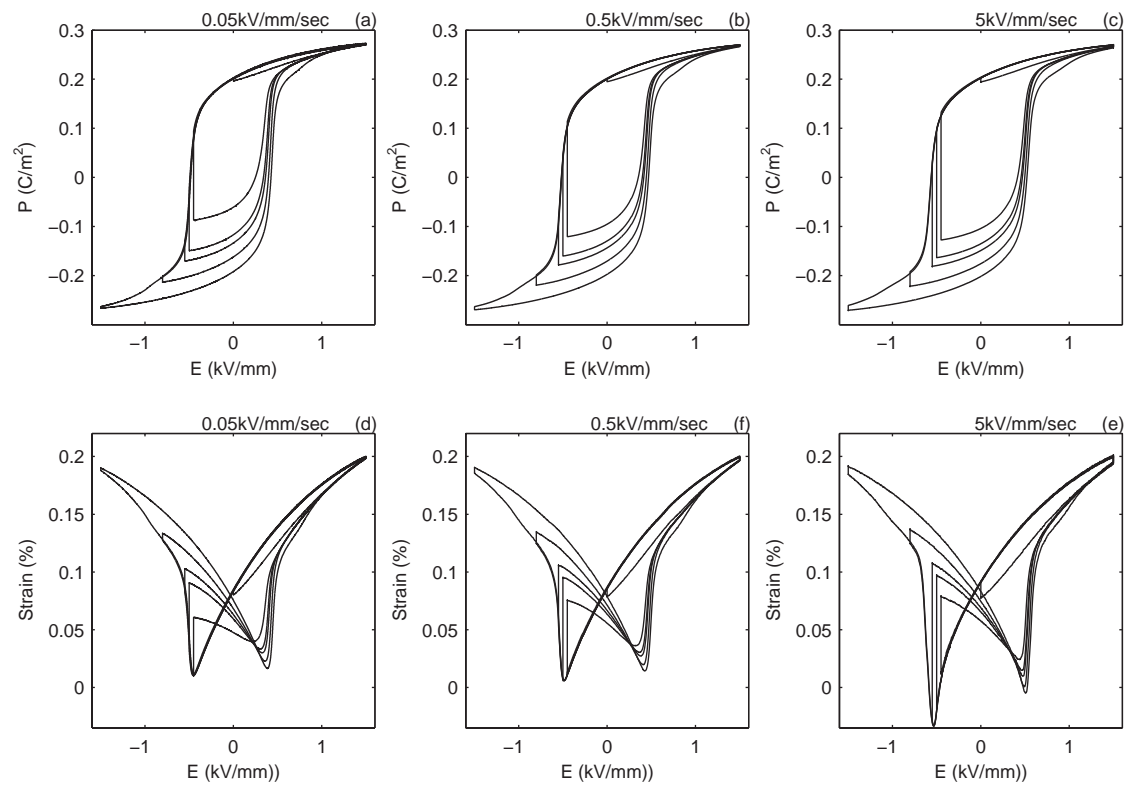

Figure 3.27: First order inner-loop hysteresis curves for creep experiments at different loading rates. (a-c) Polarizatin vs electric field. (d-f) Strain vs electric field. 
field regardless of loading rate. The strain will creep toward this equilibrium regardless of which side of the minimum it started on, as shown in subplots (f) for both figures. Also from subplots (f), the larger extensions for a shorter range of electric field reinforces that the minimum is an unstable reogon associtated with the $90^{\circ}$ chrystalline orientat.

\subsubsection{Second Order Reversals}

For the Second order reversals, rate effects, similar to those for the first order reversals, are shown in Figure 3.28. The second order reversals show very symmetric rate dependant behavior to the first order reversals rate dependence. This shows a symmetry between the rate dependant switching from the $+180^{\circ}$ orientation and switching from the $-180^{\circ}$ crystal orientation.
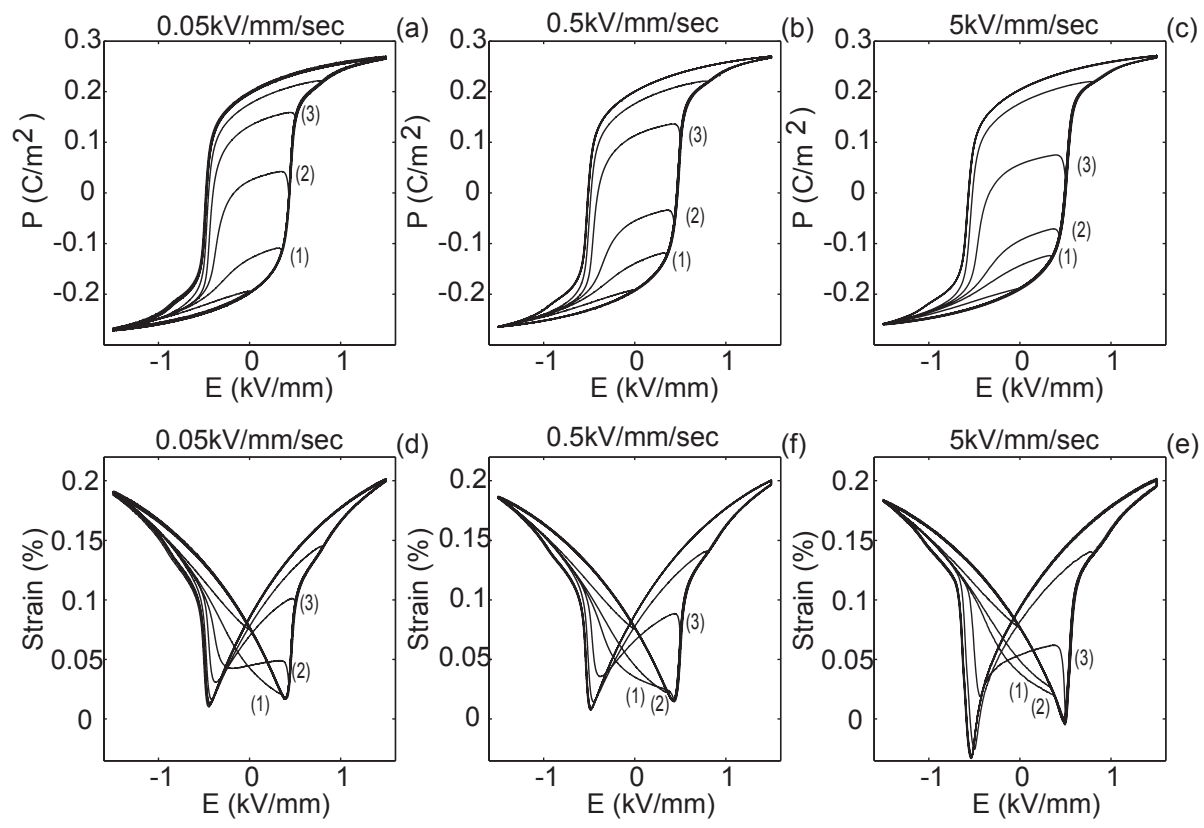

Figure 3.28: Second order reversal hysteresis curves at different loading rates. (a-c) Polarizatin vs electric field. (d-f) Strain vs electric field. 


\subsubsection{Third Order Reversals}

For the third order reversals, rate effects, similar to those for the first order reversals, are shown in Figure 3.29. The strain vs electric field hysteresis, in subplots (d-f), show the movement of the reversal loops with an increase in loading rate. The direction of travel for the loops, clockwise or counterclockwise, in subplot (d), the lowest rate changes from counterclockwise to clockwise as the reversal point moves further down the hysteresis path. When the loading rate is increased in subplots (e) and (f), the reversal points move and thus the loops themselves move. As the loop position moves to the other side of the "hump", the direction of the loops change from clockwise to counterclockwise.
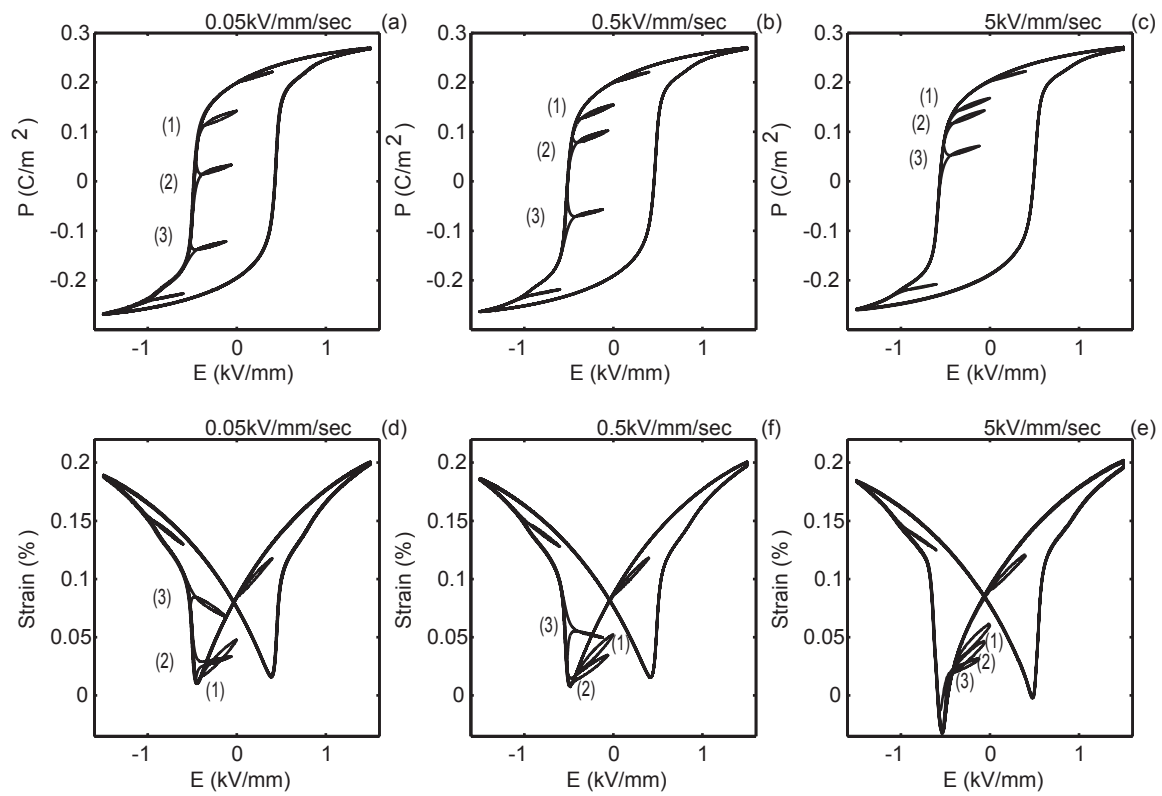

Figure 3.29: Third order reversal hysteresis curves at different loading rates. (a-c) Polarizatin vs electric field. (d-f) Strain vs electric field.

\subsubsection{Fourth Order Reversals}

For the fourth order reversals, rate effects, similar to those for the first order reversals, are shown in Figure 3.30. The fourth order reversals show very symmetric rate dependent 
behavior to the third order reversals. The same change in direction for the loop paths of the strain vs electric field reversals, subplots (d-f), that were observed for the third order reversals can been seen here for the forth order reversals.
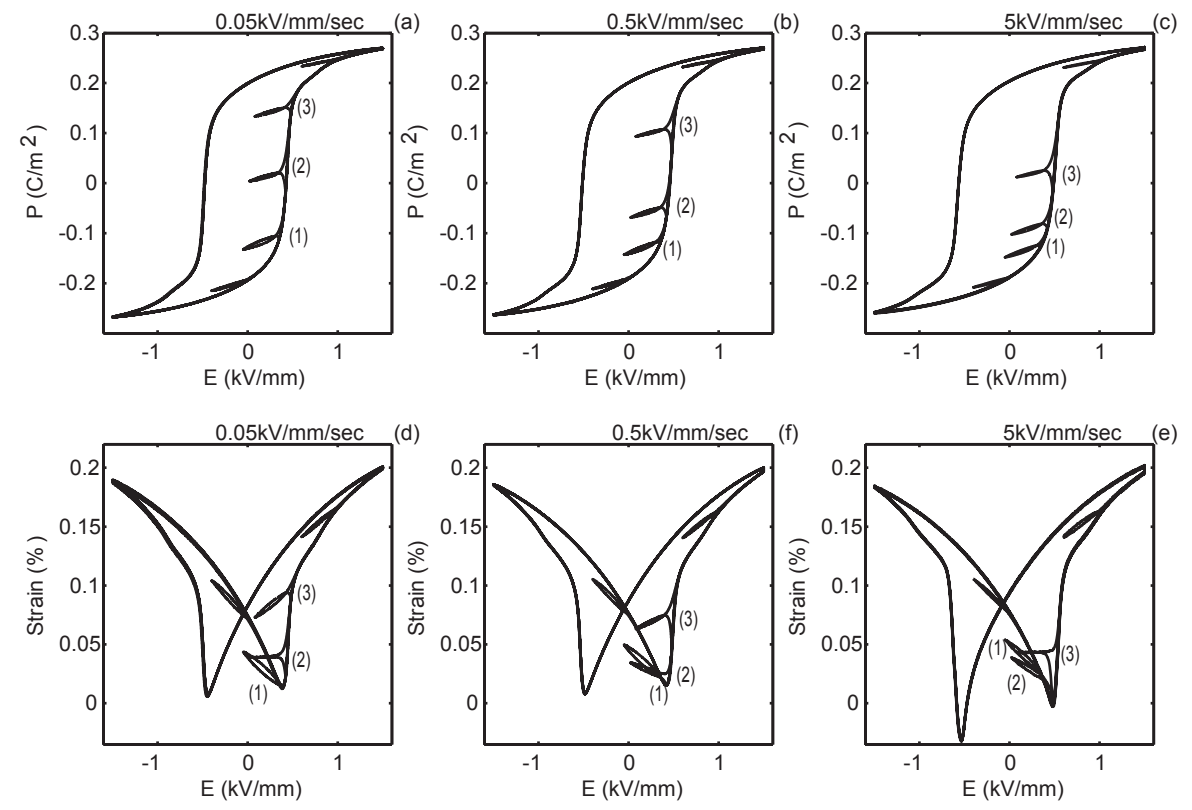

Figure 3.30: Fourth order reversal hysteresis curves for different loading rates. (a-c) Polarizatin vs electric field. (d-f) Strain vs electric field.

\subsubsection{Leakage Current Effects on Hysteresis and Creep Curves}

The leakage current, described in a previous section, was not accounted for in the measurements in this chapter. It was mentioned that the leakage current could have significant error effects on long experiments. The effects of the leakage on a polarization hysteresis plot can be analyzed by comparing the hysteresis with and without the correction for the leakage current. Figures 3.16 and ?? show long term experiments conducted for third order reversals at a loading rate of $.05 \mathrm{kV} / \mathrm{mm} / \mathrm{sec}$. The polarization hysteresis from that experiment is plotted against the corrected polarization hysteresis curve and shown in Figure 3.31 . 


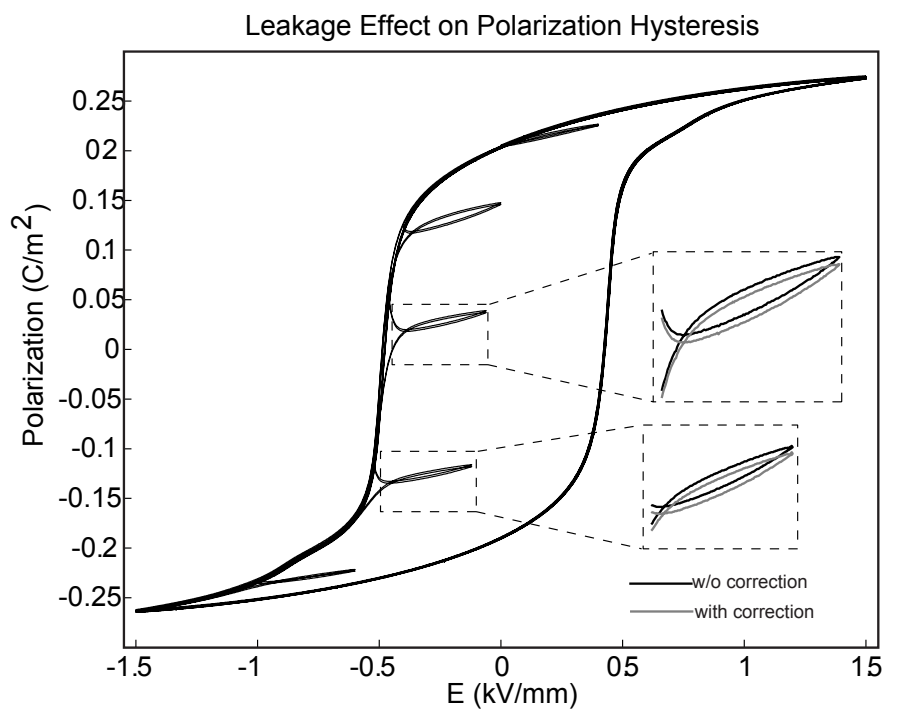

Figure 3.31: Leakage current effect on polarization hysteresis for third order reversal curves at a loading rate of $0.05 \mathrm{kV} / \mathrm{mm} / \mathrm{sec}$.

From Figure 3.31, the corrected polarization hysteresis curve and the hysteresis curve containing the leakage error are indistinguishable without the zoom. The leakage current does cause some deviation from the real hysteresis curve but the shape of the hysteresis has not changed and the error is an offset shown in the zoom. Figure 3.32 shows the leakage effects on polarization measurement vs time plots of experiments for creep behavior observation. The curve with the leakage error is plotted against the curve that has the leakage error corrected. The differences between the two are more apparent than for the hysteresis curve and just as for the hysteresis curve the shape of the creep curve remains the same. These comparisons show that the leakage current will produce a significant error in the polarization measurement although it will not significantly change the shapes of the curves. The leakage error should be taken into account if exact measurements are the goal of the experiments. The leakage is therefor a source of error in our data, but it does not significantly effect the arguments made in this thesis concerning the hysteresis shapes. 


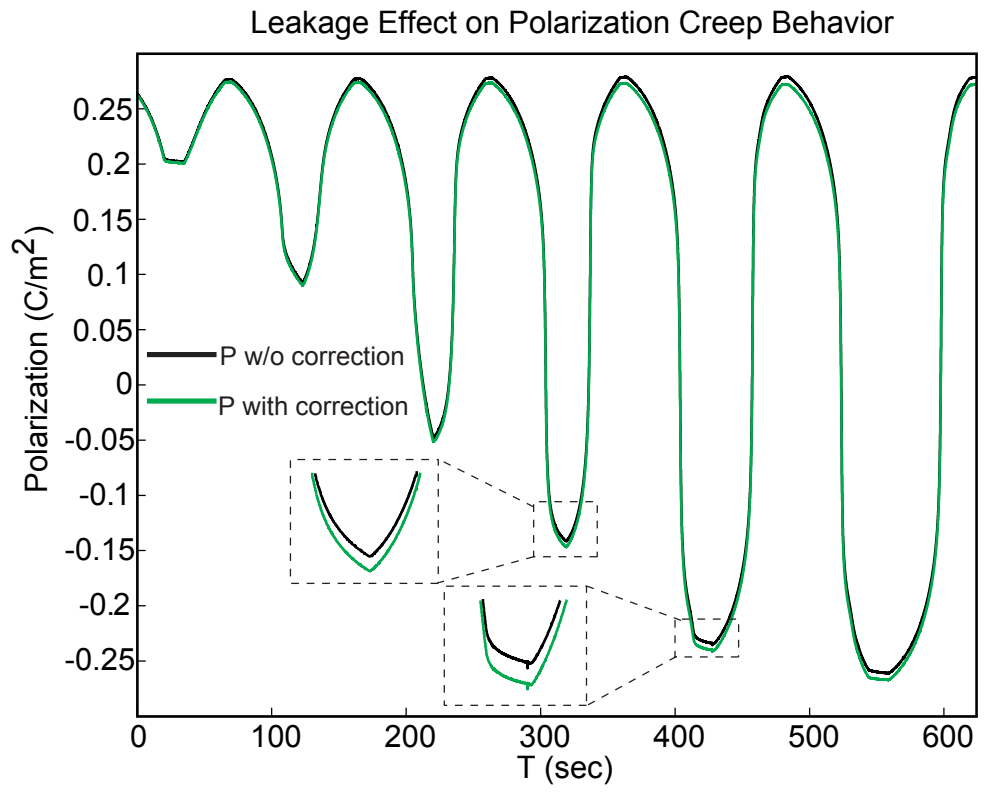

Figure 3.32: Leakage current effect on polarization creep behavior at a loading rate of $0.05 \mathrm{kV} / \mathrm{mm} / \mathrm{sec}$. 


\subsection{Conclusions}

In this chapter we have presented experimental results at different rates for the full outer hysteresis loop and four types of minor-loops in the full-loop range. From these experiments, we have observed that

1. Minor-loops have very different shapes, depending on the location of reversal.

2. Reversals that occur when the majority of the crystalline domains are oriented in the $90^{\circ}$ phase show a slower reorientation process than for reversals that occur when the majority of the crystalline domains are oriented at $\pm 180^{\circ}$.

3. Strain and Polarization have different creep behavior at different held electric field values, and the creep effect increases as the concentration of crystalline in the $90^{\circ}$ phase orientation increases.

4. As the Loading Rate for the electric field increases, the hysteresis of full-loop and minor-loops change showing a significant rate dependence.

5. Rate dependence is shown to be more significant and observable when $90^{\circ}$ reorientations of the domains are occurring, suggesting that the $90^{\circ}$ reorientation of domains is a slower process than the $\pm 180^{\circ}$ reorientation of domains. 


\section{Chapter 4}

\section{Modeling of Energy}

In this chapter, we introduce a free energy model including $90^{\circ}$ domain switching for the piezoelectric material. The model will help illustrate the hysteretic behavior of the piezoceramic presented in the previous chapters. The model will produce the Helmholtz free energy as a function of polarization. To illustrate the dynamic behavior of the domain switching process, the Gibbs free energy will be incorporated into the model and simulations run against the experimental data.

\subsection{Energy Model for Single Crystal PZTs}

One of the central goals of this thesis is to model the domain switching behavior of piezoceramics. Therefore, the model must be based on physics as closely as possible, while being computationally simple enough for future modeling applications. A mesoscopic onedimensional energy model proposed by Smith and Seelecke [27] was developed with the assumption that dipoles in a single crystal with uniform lattice spacing have two orientations, which are denoted by $(+)$ and $(-)$, depending on whether the $T i^{4+}$ ion is displaced upward or downward with respect to the center of symmetry (see Figure 2.3). This chapter will expand on that two phase model to include a $90^{\circ}$ phase variance.

For a typical mesoscopic domain consisting of a number of equally poled lattice cells, the model assumes the following multiparabolic representation for the Helmholtz free energy density $\psi$ as a function of the polarization $P$. The full piecewise function consists of five parabolas, of the form: $\psi_{i}(P)=a_{i} P^{2}+b_{i} P+c_{i}, i=1 . .5$, with different boundary conditions. 
Using those boundary and continuity conditions, as well as physical parameters extracted from the experimental data, the following Helmholtz free energy density $\psi$ as a function of the polarization $P$ is formed:

$$
\psi(P)=\left\{\begin{array}{cl}
\frac{1}{2} E p P^{2}+E p P s P+\frac{1}{2} E p P s^{2} & , P \leq-P_{180} \\
-\frac{1}{2}\left[\frac{\left(-E p P_{180}^{2}+E p P_{180} P_{90}-E p P s P_{90}+E p P s^{2}-2 E_{90}\right)}{\left(P_{180}-P_{90}\right) P_{180}} P^{2}\right. & \\
+\frac{\left(-2 P_{180} E p s+2 E p P s^{2}-4 E_{90}\right)}{\left(P_{180}-P_{90}\right)} P & ,-P_{180} \leq P \leq-P_{90} \\
\left.+\frac{\left(-2 P_{180} E_{90}-P_{90} E p P s P_{180}+P_{90} E p P s^{2}\right)}{\left(P_{180}-P_{90}\right)}\right] & \\
\frac{1}{2} \frac{\left(-P_{180} E p P s+E p P_{180} P_{90}-2 E_{90}-E p P s P_{90}+E p P s^{2}\right)}{\left(P_{180} P_{90}\right)} P^{2}+E_{90} & ,-P_{90} \leq P \leq P_{90} \\
-\frac{1}{2}\left[\frac{\left(-E p P_{180}^{2}+E p P_{180} P_{90}-E p P s P_{90}+E p P s^{2}-2 E_{90}\right)}{\left(P_{180}-P_{90}\right) P_{180}} P^{2}\right. & \\
-\frac{\left(-2 P_{180} E p s+2 E p P s^{2}-4 E_{90}\right)}{\left(P_{180}-P_{90}\right)} P & , P_{90} \leq P \leq P_{180} \\
\left.+\frac{\left(-2 P_{180} E E_{90}-P_{90} E p P s P_{180}+P_{90} E p P s^{2}\right)}{\left(P_{180}-P_{90}\right)}\right] & \\
\frac{1}{2} E p P^{2}-E p P s P+\frac{1}{2} E p P s^{2} & , P \geq P_{180}
\end{array}\right.
$$

In equation (4.1), $P_{S}, P_{180}, P_{90}$ and $E_{P}$ are related to physical properties, which are found directly from the polarization vs electric field single crystal hysteresis curve fitted to the experimental data as shown in Figure 4.1. $P_{S}$ denotes the remnant polarization, $P_{180}$ the onset of the switching process from $180^{\circ}$ into $90^{\circ}, P_{90}$ the onset of the switching process from $90^{\circ}$ into $180^{\circ}$, and $E_{p}$ is the reciprocal of the slope of the $(E, P)$ curve.

The Helmholtz free energy density $\psi$ as a function of the polarization $P$ is plotted in Figure 4.2. The parameter values are extracted from experimental data with rough approximations to the single crystal model.

The switching processes in the material can be interpreted as phase transformations with the polarization as the order parameter. The kinetics of these phase transformations are determined by the electric-field-dependent barriers and minima in the Gibbs free energy, which is related to the Helmholtz free energy in equation (4.1) through the Legendre transformation $G=\psi-E P$. This dependence is illustrated in Figure 4.3 for increasing 


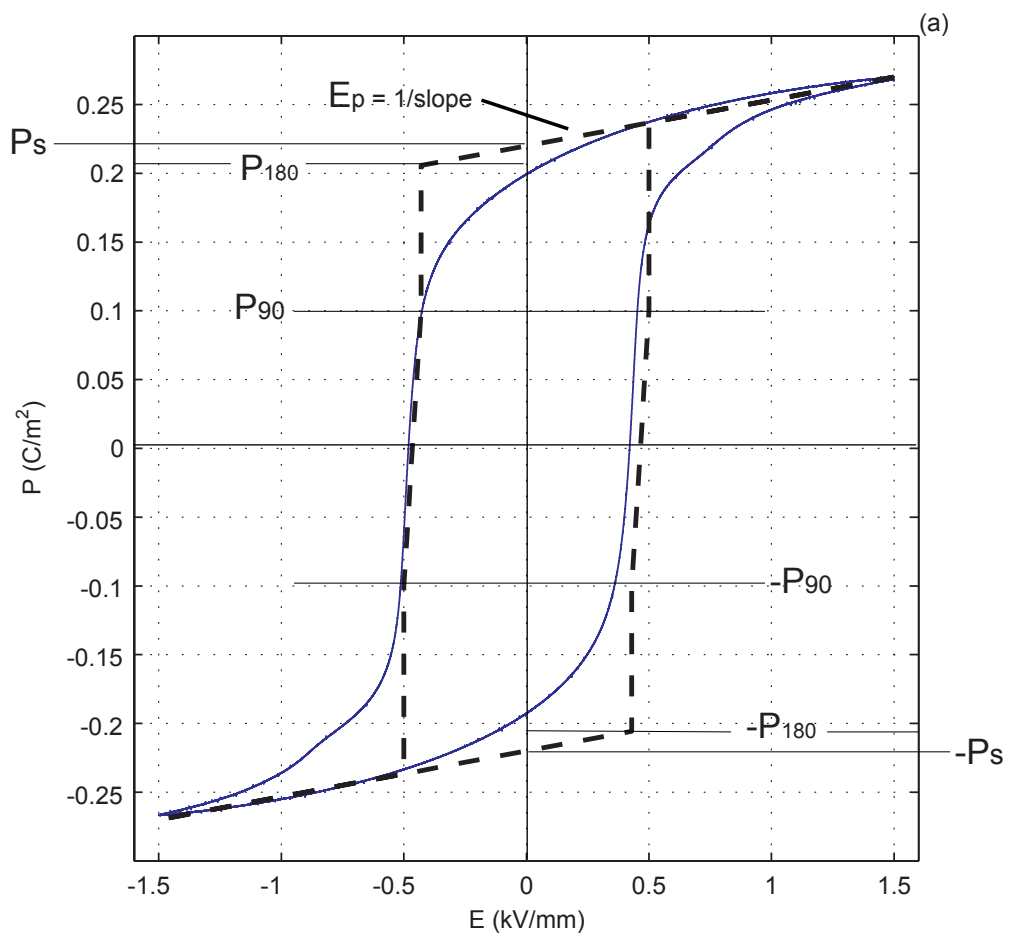

Figure 4.1: Polarization vs electric field for experimental data fitted with a single crystal approximation curve. Dashed line is theoretical single crystal curve, while solid curve is experimental curve. 


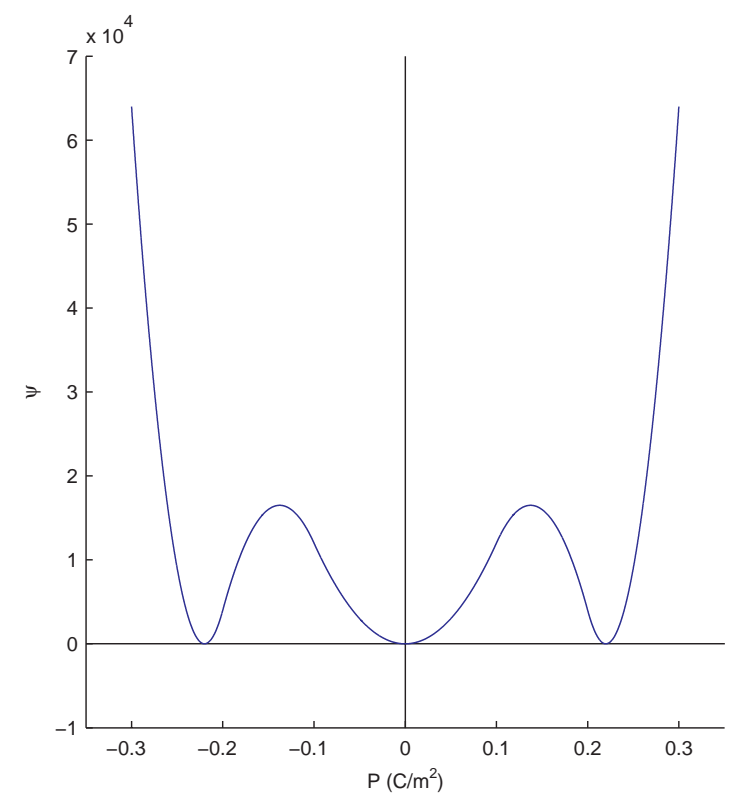

Figure 4.2: Helmholtz free energy as a function of polarization.

field values (from left to right starting at $\mathrm{E}=0$ ) together with the corresponding polarization vs. electric-field behavior (Polarization starting at fully poled negative $180^{\circ}$ ). The dark ball indicates the value of the polarization for the single crystal at a particular time which changes as the electric field is increased (left to right).

Since our experiments are not conducted with a single crystal, we must build a model that contains many different crystals with different orientations and that will switch orientations at different potentials of electric field. In order to illustrate a large number of crystals, we place several balls into the energy wells and give them all a distribution of initial kinetic energy and allow each one to switch at different energy values which mimics the different energy barriers seen by the actual crystals in the material. A simulation is performed that gives each crystal, represented by a small ball, its own freedom to move through the energy model. Because they each have different energy criterion, some will switch orientations sooner than others creating the smooth hysteresis curve found in the experimental results. 

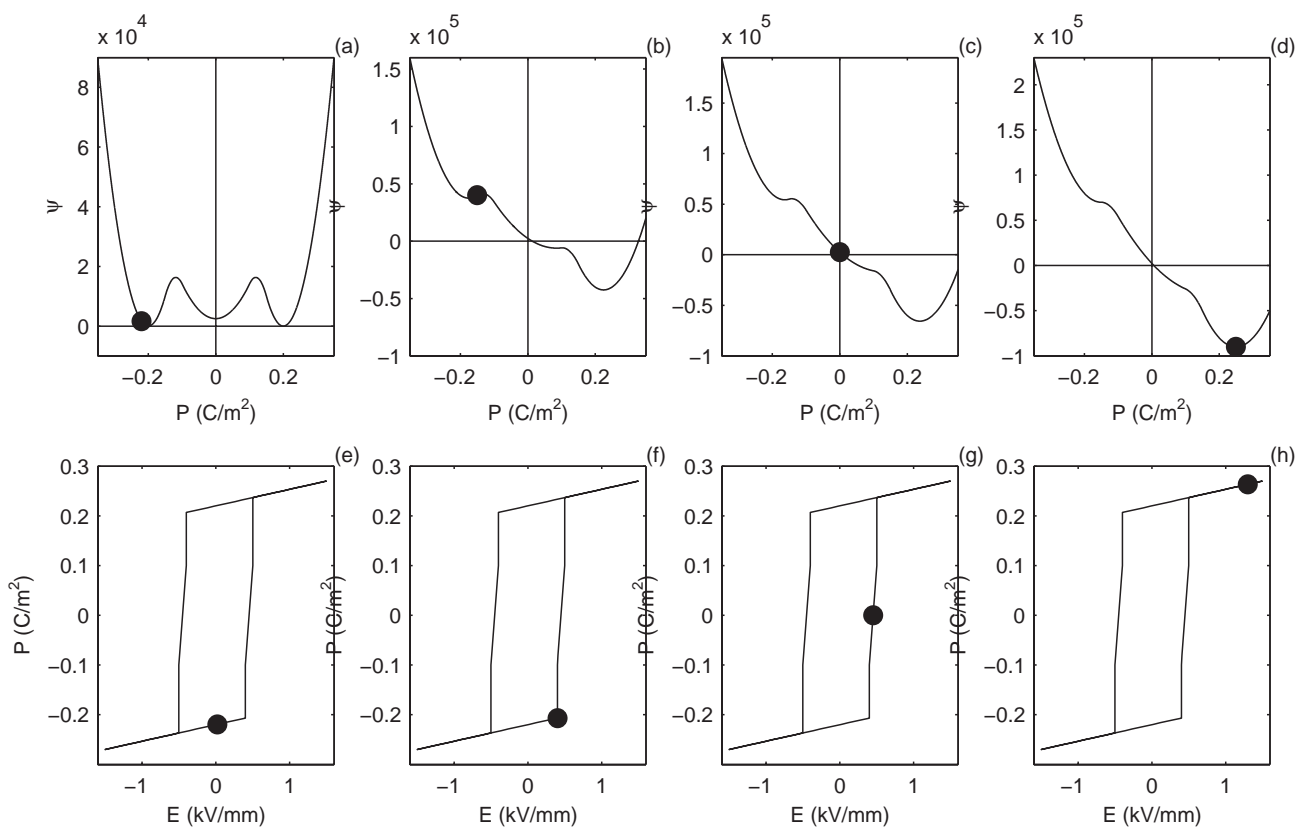

Figure 4.3: Gibbs energy for a single crystal as a function of polarization with increasing electric field (a-d), and the corresponging single crystal polarization vs electric field (e-h). 
The actual polarization value is the net charge of all the individual crystallites' polarizations. Figure 4.4 shows snapshots of the energy model vs polarization simulation along with experimental data for full loop hysteresis linearly loaded at $0.05 \mathrm{kv} / \mathrm{mm} / \mathrm{sec}$. The time progress starts with subfigure (a) and ending with subfigure (f). Each subfigure shows, starting from upper left going clockwise, the prescribed electric field, the energy model, the polarization vs electric field, and the strain vs electric field. The model based on Gibbs energy is prescribed the equivalent electric field to the experimental data. The energy model has 100 balls moving through the phases independently following prescribed physical constraints. The average polarization of those balls is posted with the energy model plot. The large balls on the hysteresis curves and proscribed energy curve show the time point for the experimental data corresponding to the prescribed electric field, strain, and polarization values.

From Figure 4.4, the model simulation illustrates the domain switching process as it occurs for particular values of the electric field over time(a-f). Figure 4.5, shows a model simulation for a full loop linearly loaded at $5 \mathrm{kV} / \mathrm{mm} / \mathrm{sec}$.

When the $0.05 \mathrm{kV} / \mathrm{mm} / \mathrm{sec}$ loading rate simulation in Figure 4.4 is compared to the faster $5 \mathrm{~V} / \mathrm{mm} / \mathrm{sec}$ loading rate simulation in Figure 4.5 fraem for frame, the delayed switching process can be seen in sublots (c) and (e) for each of the rates. More balls have begun to switch over for the slower rate than for the faster rate. This illustrates that the switching behavoir is rate dependant and higher loading rates will result in delayed switching.

Figure 4.6 uses the model to illustrate the domain switching at first order reversal points. The rounding effects that where shown earlier are shown here to correspond to higher concentrations of $90^{\circ}$ crystal orientations. The model plots show a large amount of balls in the $90^{\circ}$. energy well corresponding to a $90^{\circ}$ orientation. When the electric field is reversed, these balls will for a short time continue to move toward the $-180^{\circ}$ phase since they have already overcome the first energy wall and now have some momentum toward the $-180^{\circ}$ phase.

From Figure 4.6, the position of the balls at the reversal points illustrates the switching behavoir of the piezoceramic actuator. The hysteresis behavoir found in the experiments resulting from the slower $90^{\circ}$ reorintation of the materials domains matches the predictions of the simulations. 
This simulation produces short animations that better demonstrate the domain switching behavior. Those animations of course are not able to be produced in this written thesis. The simulation in its current state however is only a illustrative tool and would not work for applications. One of the reasons for this is that the model is only guessing at the switching rates of the crystals based on experimental observation. The speed at which the balls move through the energy model is approximated and are programed to match the expectations from the experimental data. The ball motions are based on three parameters: (1) its thermal kinetic energy (small). (2) the applied electric field, and (3) its current polarization value. The exact effects of these three parameters on the switching rates were not able to be extracted from the experimental data, so the simulation uses a complex algorithm based on those parameters to estimate the balls changing motion. Future analysis and experimentation may allow for the extraction of those values and allow the program to generate a more accurate and control oriented simulation. For this thesis, the simulation is only designed to give the reader a better illustration and explanation for the domain switching process. 


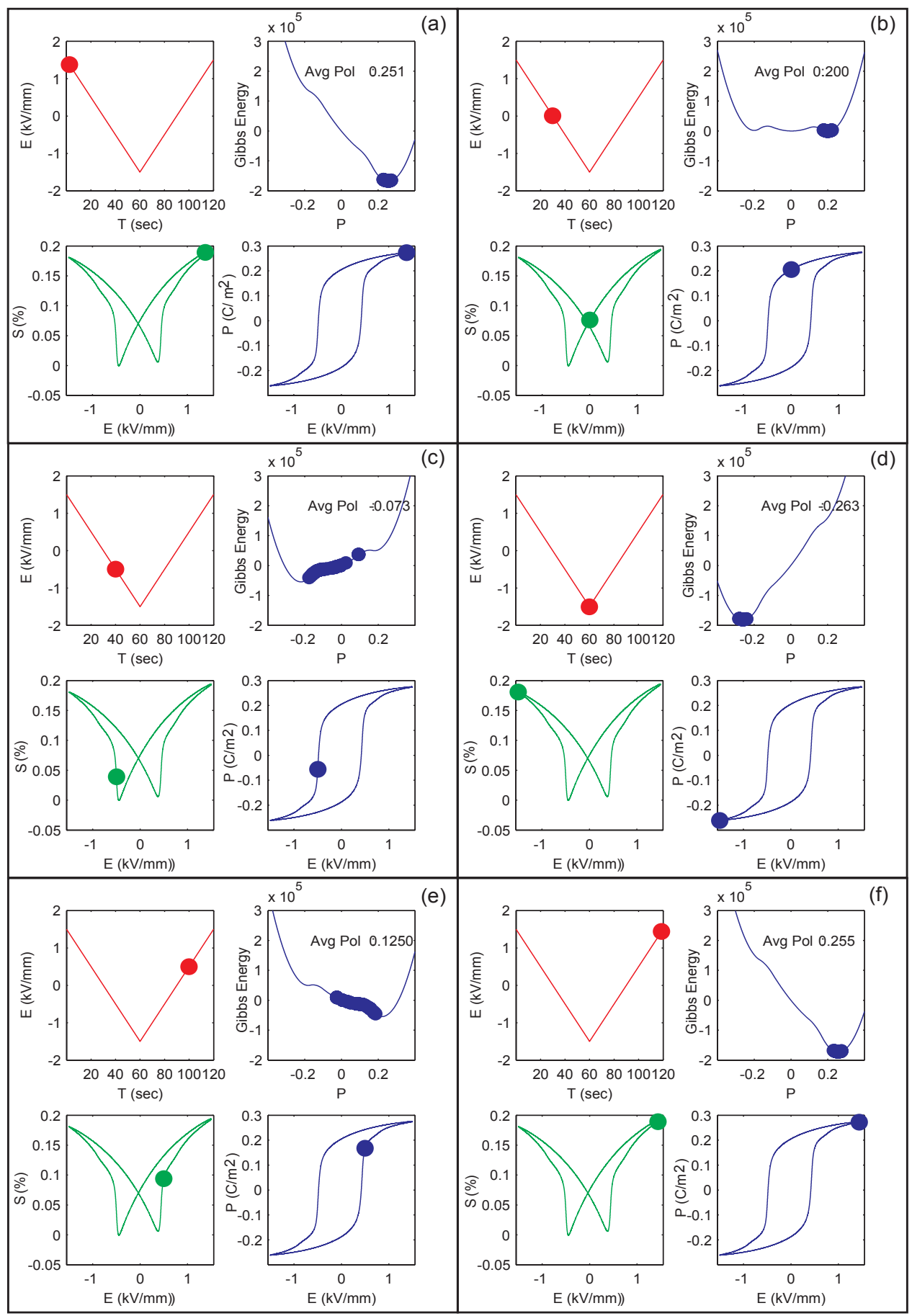

$\sim$

Figure 4.4: Simulation of switching behavior for full-loop hysteresis at a loading rate of $0.05 \mathrm{kV} / \mathrm{mm} / \mathrm{sec}$. 


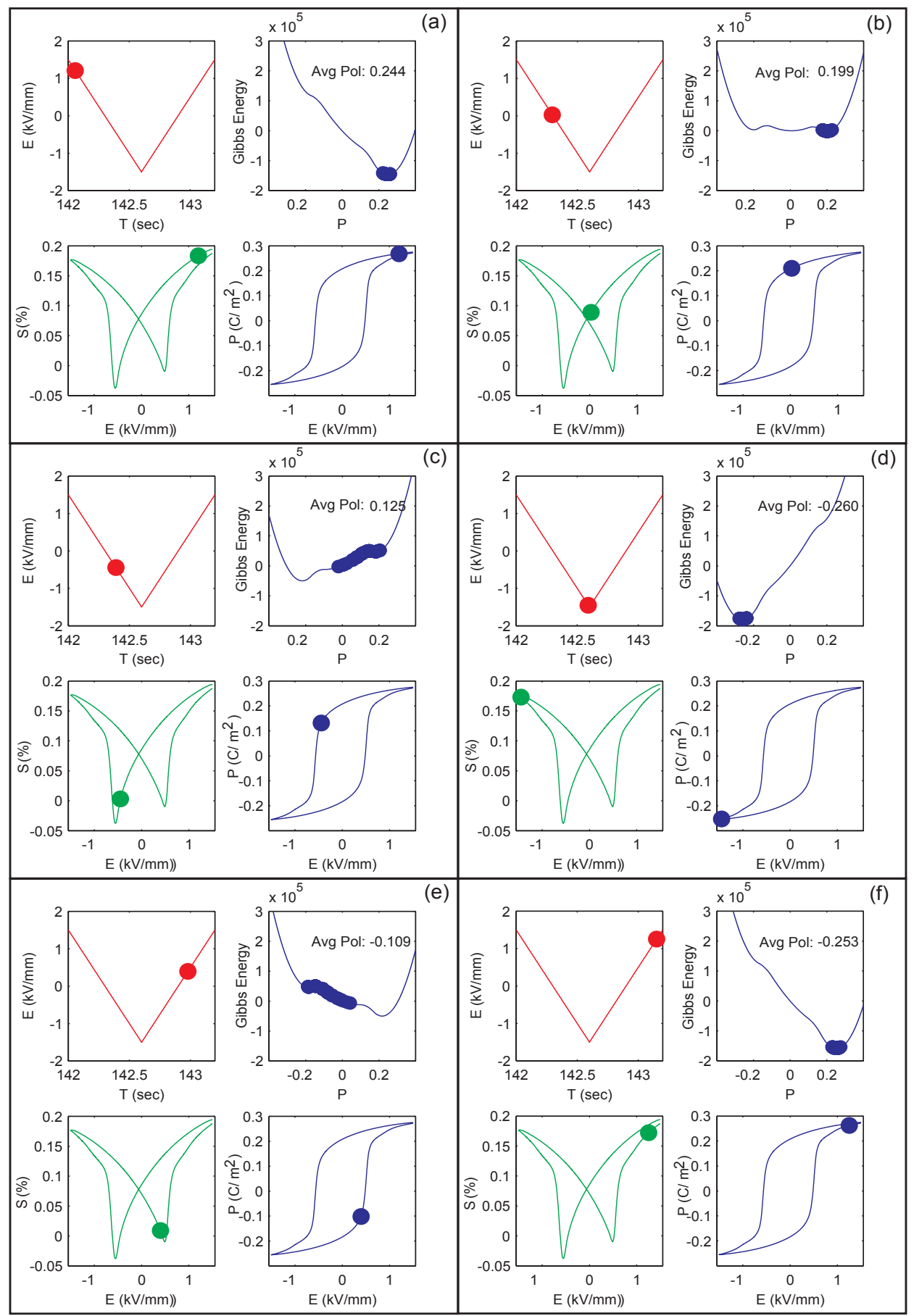

Figure 4.5: Simulation of switching behavior for full-loop hysteresis at a loading rate of $5 \mathrm{kV} / \mathrm{mm} / \mathrm{sec}$. 


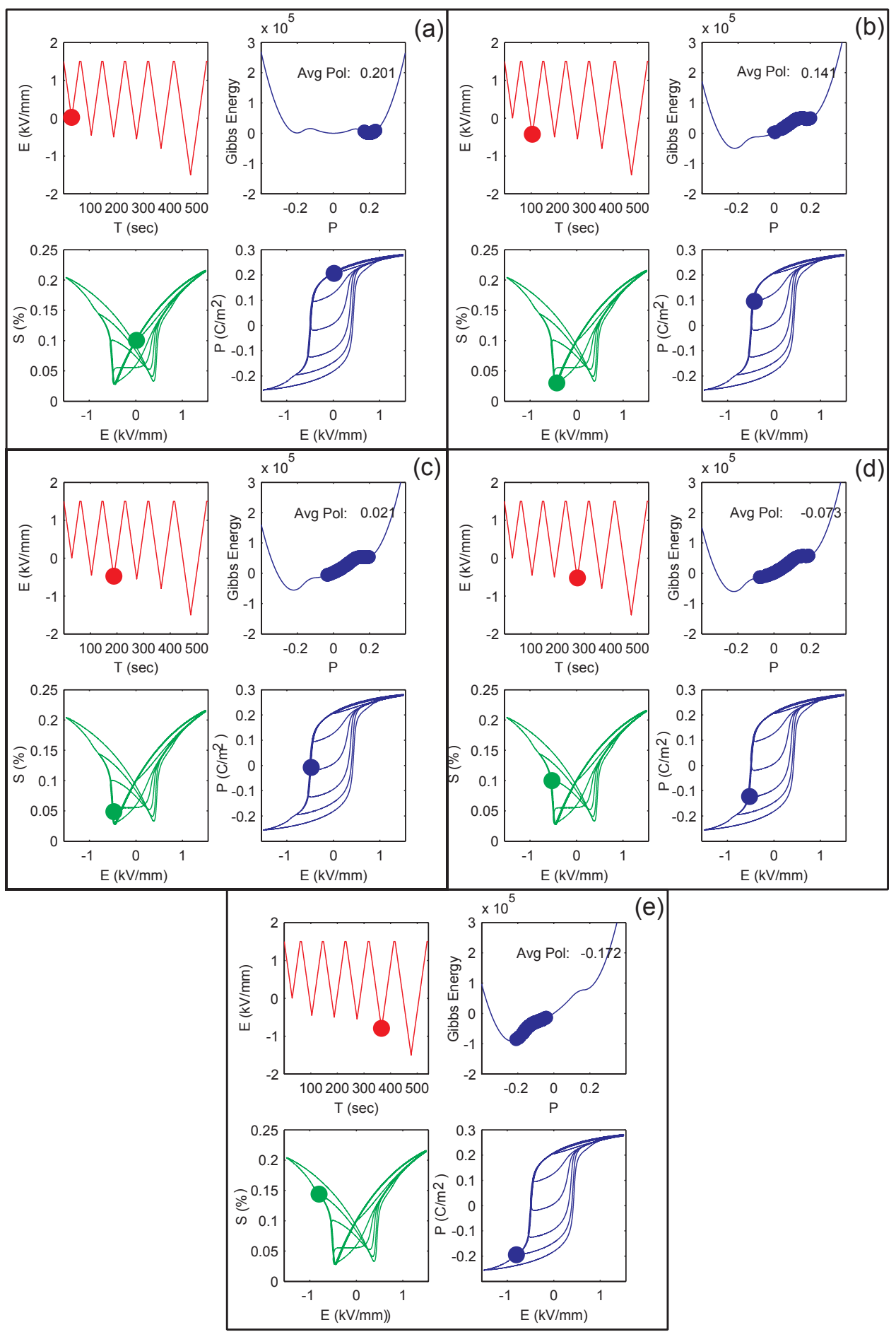

Figure 4.6: Simulation of switching behavior for first order reversals of inner-loop experiments at $0.05 \mathrm{kV} / \mathrm{mm} / \mathrm{sec}$. 


\section{Chapter 5}

\section{Conclusions}

In this thesis, the rate dependant hysteresis of a PZT actuator was investigated. The role of domain switching within the material was investigated and an energy based graphical model of the domain switching process was introduced. The thesis was presented in several parts.

First, several experiments were conducted on a commercially available soft PZT stack actuator at relatively low-rate piecewise linear electrical loading. The hysteretic behavior was observed during first, second, third and fourth order reversals. These minor loop experiments showed unusual behavior for the polarization and strain when the electric field was reversed at a point where high concentrations of crystallites were undergoing $90^{\circ}$ domain switching. The shapes of the hysteresis curves became rounded, and the polarization curves did not reverse direction as quickly as the electric field was reversed. This suggests that the $90^{\circ}$ variant has a longer switching time than the $\pm 180^{\circ}$ variants. Crystallites that have started to switch into the $90^{\circ}$ variant immediately will not jump back into the $+180^{\circ}$ at the time of the reversal, instead they continue on toward the $-180^{\circ}$ variant causing the polarization and strain to continue toward their current direction beyond the reversal point.

Second, using the same actuator, experiments were conducted that focus on the ratedependent behavior of the material. Different loading rates were used for the same full-loop and minor-loop experiments conducted in the first part. For the full-loop experiments, we found the polarization curves to widen, showing a delayed reaction in response to the electric fields faster rate due to the inertia of the switching process. The strain also had a delayed 
reaction and was able to achieve a longer stroke. The delayed reaction was, similarly to the polarization, caused by the delayed domain switching. The longer stroke was caused by domains quickly switching out of either $\pm 180^{\circ}$ variants and into the $90^{\circ}$ variants, while the reorientation out of the $90^{\circ}$ variant was relatively slower. This resulted in more crystallites being in the $90^{\circ}$ variant at the same time. The shorter stroke results because the $90^{\circ}$ orientation produces the shortest length of each crystal along the $\mathrm{d}_{33}$-axis (along the axis that the electric field is applied and along which the strain is measured).

We also investigated the creep behavior by holding the electric field constant at different levels within the full loop range. The creep behavior was observed to be greater in regions concentrated in $90^{\circ}$ crystallite variant orientations. This confirmed that the $90^{\circ}$ variants undergo a slower reorientation process than the $\pm 180^{\circ}$ variants.

The fourth part of this thesis used Helmholtz and Gibbs free energy relations to model the domain switching of the material. A simulation model was developed that shows domain switching under prescribed electrical fields. Based on the observed difference in relaxation times, the simulation was able to illustrate the domain switching precesses that led to the hysteretic behavior found during experimentation.

Future work for this research involves improving the Gibbs free energy model to accurately simulate the materials behavior. The data collected from these experiments will be used to more accurately formulate future models as well as serve as a standard for comparison against simulations. 


\section{List of References}

[1] J. Zhong. Modeling and Control of Piezoceramic Actuators for Nano-positioning. Masters thesis, North Carolina Stat University, Raleigh, NC USA, 2003..

[2] M.A.Weber, M. Kamlah, D.Munz. Experiments about time effects in piezoceramics(in German). Technical Report FZKA 6465, Forschungszentrum Karlsruhe GmbH, Karlruhe, Germany, 2000.

[3] D. Zhou. Experimental Investigation of Non-linear Constitutive Behavior of PZT Piezoceramics. PhD thesis, Forschungszentrum Karlsruhe GmbH, Karlruhe, Germany, 2003.

[4] K. Dragosits. Modeling and Simulation of Ferroelectric Devices. PhD thesis, Technischen Universit at Wien, 2000.

[5] B.K. Mukherjee, S. Sherrit, Characterization of Piezoelectric and Electrostrictive Materials For Acoustic Transducers: II. Quasistatic Methods. Fifth International Congress on Sound and Vibration, December 15-18, 1997.

[6] F. Li, D. Fang, Simulations of domain switching in ferroelectrics by a three-dimensional finite element model, Mechanics of Materials 36, 2004, p959-973.

[7] S.C. Hwang, C.S. Lynch, R.M. McMeeking, Ferroelectric/ferroelastic interactions and a polarization switching model., Acta Metallurgica et Materialia 45, 2073. 1995.

[8] F. Preisach, Zeitschrift fur Physik. 94:277-302, 1935

[9] Y. Bernard, E. Mendes, F. Bouillault. Dynamic Hysteresis Modeling Based on Preisach Model. IEEE Transactions on Magnetics, Vol. 38, No. 2, March 2002. 
[10] I.D. Mayergoyz, G. Friedman, C. Salling. Comparison of the classical and generalized Preisach hysteresis models with experiments. Magnetics, IEEE Transactions on , Volume: 25 , Issue: 5:3925 - 3927, Sep 1989

[11] I.D. Mayergoyz. Hysteresis models from the mathematical and control theory points of view. J. Appl. Phys. 57(1), 1985.

[12] H. Jung, J.Y.Shim, D. Gweon, Tracking Control of piezoelectric actuators, Nanotechnology 12, 14-20, 2001

[13] P. Ge, M. Jouaneh, Tracking Control of a Piezoceramic Actuator, IEEE Transactions on Control Systems Technology, Vol. 4., NO. 3, May, 1996

[14] W. T. Ang, F. A. Garmon, P. K. Khosla, C. N. Riviere, Modeling Rate-dependant Hysteresis in Piezoelectric Actuators., Proceedings of the 2003 IEEE/RSJ Intl. Conference on intelligent Robotics and Systems. Oct. 2003.

[15] R. C. Smith, Z. Ounaies, R. Wieman, A Model for Rate-dependant Hysteresis in Piezoceramic Materials Operating at Low Frequencies. ICASE Report No. 2001-26, 2001.

[16] R. C. Smith, A. Hatch, T. De, Model Development for Piezoceramic Nanopisitioners., Proceedings of the 42nd IEEE Conference on Decision and Control, 2003.

[17] C.T. Sun, L.Z. Jiang, Domain switching induced stress at the tip of a crack in piezoceramics. Proceedings of the 4th ESSM and 2nd MIMR Conference, Harrogate, p715, 1998.

[18] Y. Fotinich, G.P. Carmen, Nonlinear behavior of polycrystalline piezoceramics. Proceedings of SPE, Smart Structures and Materials, Active Materials: Behavior and Mechanics, vol 3992, p319, 2000

[19] S.C. Hwang, G. Arlt, Switching in ferroelectric polycrystals. Journal of Applied Physics, Vol 87, Num 2, pp.869-875, 2000.

[20] S.C. Hwang, J. E. Huber, R. M. McMeeking, N.A. Fleck, The simulation of switching in polycrystalline ferroelectric ceramics. Journal of Applied Physics, Vol 84, Num 3, pp.530-1540, 1998. 
[21] H. Richter, E.A. Misawa, D.A. Lucca, H. Lu. Modeling nonlinear behavior in a piezoelectric actuator. Journal of the International Societies for Precision Engineering and Nanotechnology, pp.128-137, 2001.

[22] Y-C. Huang; D-Y. Lin. Tracking control of a piezoelectric actuator based on experiment hysteretic modeling. Advanced Intelligent Mechatronics, 2003. AIM 2003. Proceedings. 2003 IEEE/ASME International Conference on , Volume: 1, pp.308 313,2003

[23] H.J.M.T.S. Adriaens, W.L. De Koning, R. Banning. Modeling piezoelectric actuators. Mechatronics. IEEE/ASME Transactions on , Volume: 5, Issue: 4, pp.331-341, 2000

[24] P. Krejci, K. Kuhnen. Inverse control of systems with hysteresis and creep. Control Theory and Applications, IEE Proceedings- , Volume: 148 , Issue: 3 , pp.185 - 192, 2001 .

[25] Y. Stepanenko, S. Chun-Yi. Intelligent control of piezoelectric actuators. Decision and Control. Proceedings of the 37th IEEE Conference on , Volume: 4 , pp.4234 - 4239. 1998.

[26] R.C. Smith, S. Seelecke, Z. Ounaies. A free energy model for piezoceramic materials. In Proc. 9th Ann. Int. Symp. Smart Struct. Mat., Modeling, Signal Processing and Control, San Diego, CA, U.S.Z., March 2002 SPIE.

[27] R.C. Smith, S. Seelecke, Z. Ounaies, J. Smith. A free energy model for ferroelectric materials. Journal of Intelligent Material Systems and Structures, vol 14, 11, pp.719$739,2003$.

[28] R.C. Smith, S. Seelecke, An Energy Formulation for Preisach Models, 9th SPIE Conference on Smart Structures and Materials, San Diego, USA, 17-22 March 2002.

[29] J. Fan, W. A. Stoll, C. S. Lynch. Nonlinear constitutive behavior of soft and hard PZT:experiments and modeling. Acta Materialia, Vol. 47, Iss. 17 , 26 November, pp. 4415-4425, 1999 
[30] W. Zhang, K. Bhattacharya. A computational model of ferroelectric domains. Part 1: model formulation and domain switching. Acta Materialia 53, pp.185-198, 2005.

[31] R. Bouregba, G. Poullain. Computation of the polarization due to the ferroelectric layer in a stacked capacitor from Sawyer-Tower hysteresis measurements. American Institute of Physics, 2003.

[32] http://www.physikinstrumente.com/

[33] http://hyperphysics.phy-astr.gsu.edu/hbase/solids/piezo.html

[34] http://www.piezotechnologies.com

[35] http://www.morganelectroceramics.com

[36] http://www.madcitylabs.com

[37] http://www.micromega-dynamics.com

[38] http://www.nanopositioning.com

[39] http://www.burleigh.com

[40] http://www.thermomicro.com/spmguide/contents.htm

[41] http://www.nanowave.com 


\section{Appendix A}

\section{Simulink/Matlab Files}

\section{A.1 Simulink Block Diagram}

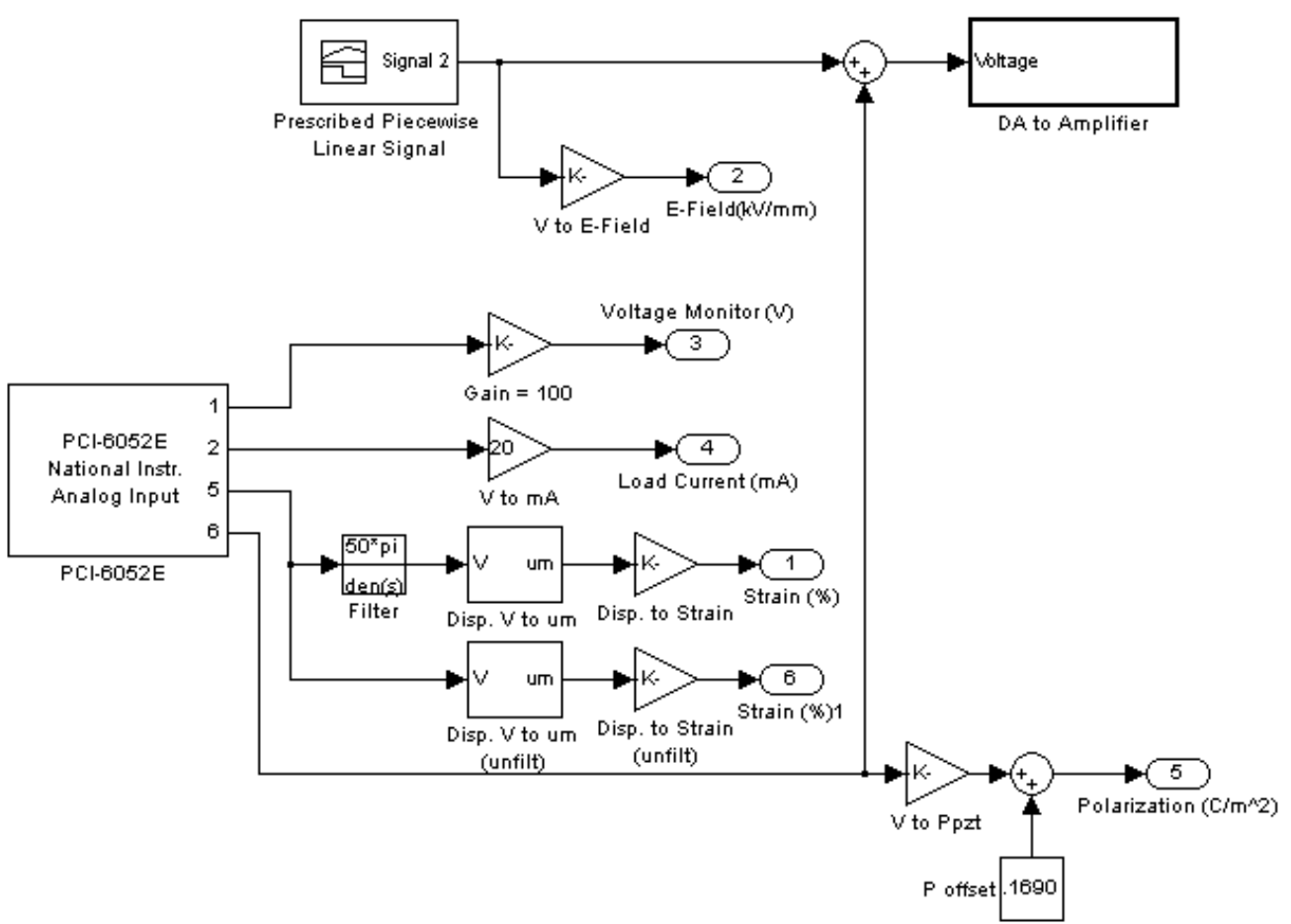

Figure A.1: Simulink Block Diagram 